\title{
Endogenous Liquidity and the Business Cycle*
}

\author{
Saki Bigio ${ }^{\dagger}$ \\ Department of Economics \\ New York University
}

February 14, 2010

\begin{abstract}
We present a model in which asymmetric information in the quality of capital endogenously determines liquidity in equity markets. Liquid equity is used as collateral for investment projects. We explain how liquidity, the price of equity and aggregate investment are jointly determined and how they interact with shocks to aggregate productivity and to the distribution of capital quality. The framework is used to decompose business cycles into real and liquidity driven. In fact, recessions can occur without changes in aggregate productivity.

We then incorporate a financial sector whose capacity to deal with asymmetric information depends on its net-worth. Financial losses occur when the the distribution of capital quality is worse than expected. As net-worth falls, financial losses exacerbate asymmetric information in future periods. Moreover, as the financial sector takes less risk, its net-worth longer to recover. Through this interaction, the liquidity channel becomes a more powerful and persistent source of aggregate fluctuations.
\end{abstract}

Keywords: Liquidity, Asymmetric Information, Business Cycles

JEL Classification: E32, E44, D82, G01, G21

*I would like to thank Ricardo Lagos and Thomas Sargent for their constant guidance in this project. I would also like to thank George-Marios Angeletos, Alberto Bisin, Roberto Chang, Mark Gertler, Nobuhiro Kiyotaki, Albert Queralto and Eduardo Zilberman for useful discussions and seminar participants of NYU third year paper seminar. All errors are mine.

${ }^{\dagger}$ Saki Bigio, Department of Economics, New York University. e-mail: sbigio@nyu.edu. 


\section{Overview}

What are the forces determining the degree of liquidity in capital markets? How is liquidity related to the business cycle? Can liquidity cause a recession without changes in aggregate productivity? This paper presents a theoretical framework where liquidity in the equity market is explained by asymmetric information in the quality of assets. Here, we define liquidity as the property of an asset by which gains from trade are sufficient to guarantee its trade. Thus, as with a lemons problem, the dispersion of capital quality segments equity into liquid and illiquid . The degree of liquidity is linked to the business cycle because liquid equity is used to relax financing constraints. Due to limited enforcement, entrepreneurs are required to input collateral in order to obtain external financing for investment projects. Liquid assets are sold in order to increase collateral. Therefore, when liquidity dries-up, there is under-investment. This inefficiency is reflected through a wedge between the price of equity and the real cost of investment. This paper is a study on how liquidity, investment and the price of equity respond together to aggregate productivity shocks and to changes in the distribution of capital quality.

In the model, the real asset backing equity is the productive capital of a firm whose identity is private information to a seller. Productive capital in the model suffers permanent idiosyncratic shocks. Since potential buyers of equity ignore the identity of an original issuer, they also ignore the actual quality of equity when they buy it. We derive the non-exclusive competitive equilibrium for this environment and show that it is one in which financial firms pool heterogeneous equity together and sell it as a homogeneous asset. The degree of liquidity is determined by a problem of adverse selection. Liquidity and the price equity are, therefore, determined jointly through a feedback effect: the degree of liquidity determines the price of equity through the demand for saving instruments. In turn, agents anticipate the price of equity, which affects the incentives to sell equity in a given pool. This market mechanism is embedded into a business cycle model with a collateral constraint on investment.

We show how recessions can occur without changes aggregate productivity through the liquidity channel. Indeed, an increase in the mean preserving spread of capital quality is enough to generate a liquidity shortage. When collateral constraints are binding, liquidity dry-ups cause investment to fall. On the other hand, we also show that the amount of liquidity is always insufficient and inefficient. The argument is simple: efficient investment requires liquidity, liquidity requires a positive wedge and investment cannot be efficient if there is a wedge in the price of equity.

Though liquidity depends on the degree of information asymmetry, liquidity crises typically occur in conjunction with a crisis within the financial sector. The recent financial crisis has not been an exception. ${ }^{1}$ To understand this relationship, we extend the core framework to incorporate a more complex financial sector. Here, we develop the concept of financial risk capacity which is a measure of the financial sector's capacity to undertake risk in markets with asymmetric information. ${ }^{2}$ Financial risk capacity is a function of the financial system's net-worth. In this extension, financial firms are also uncertain about the true distribution of capital quality. This feature makes financial profits from pooling equity an uncertain random outcome.

The dynamics of financial risk capacity introduce a novel mechanism. In essence, when the distribution of capital quality is worse than expected, financial firms face liabilities which they only finance by liquidating part of their net worth. On the other hand, with a lower net worth, financial firms have a lower buffer-stock to finance losses in future poor states. Therefore, they are forced to undertake less risk ex-ante when pooling equity. This means that they offer worse terms to sellers

\footnotetext{
${ }^{1}$ For example, Reinhart and Rogoff and Brunnermeier [2009] provide a narrative description of the events and associate them with asymmetric information and shocks to the financial sectors position.

${ }^{2}$ Bigio [2010a] explores several government policies for a simpler version of this model.
} 
of equity which exacerbates the problem of asymmetric information. Lower financial risk capacity therefore implies less liquidity together with stronger and more prolonged recessions.

Why should we care about an explaining liquidity through asymmetric information? Why not limit to an environment in which liquidity is determined exogenously? Studying asymmetric is important to understand liquidity because it imposes restrictions on the amount of liquidity. For example, the productivity of capital improves the degree of liquidity. This explains why liquidity should eventually recover after a recession as the capital stock falls. Thus, we should expect a positive correlation between liquidity shocks and the return to capital. Another difference is that models with exogenous liquidity may allow complete market shut-downs. In contrast, under asymmetric information, full market shutdowns require a restriction on the distributions of capital quality which can be qualified with data. On the other hand, in this environment liquidity may never be sufficiently high to make in this environment.

More so, endogenous liquidity provides immunity against the Lucas critique. A number of recent papers, following similar lines as Kiyotaki and Moore [2008] (henceforth, KM), ${ }^{3}$ study the role of government interventions that target the degree of liquidity in equity markets. By abstracting from the causes of liquidity dry-ups, these papers cannot offer answers on how agents respond to these policies. The reaction of the private sector may be important to address questions about the implementation and welfare implications of such policies.

This paper follows prior work by KM who study a static model where exogenous shocks affect the degree of liquidity. KM are the first authors to outline the channel by which liquidity affects investment. We follow them in combining limited enforcement with the dynamic effects of liquidity but we provide an endogenous mechanism to explain liquidity. Asymmetric information in capital markets has been broadly studied since the seminal contribution of Stiglitz and Weiss [1981]. ${ }^{4}$. However, there is little work in a general equilibrium framework. A notable exception is Eisfeldt [2004] who studies how liquidity is determined by asymmetric information in an economy without capital accumulation. As opposed to us, the stress of that paper is on explaining how liquidity affects risk sharing and abstracts from relating liquidity to aggregate shocks.

Kurlat [2009] independently develops a similar environment to the one presented here in order to explain how liquidity affects aggregate output along the cycle. That paper introduces a continuum of shocks to the technical rate of transformation of investment and consumption goods (TRT) whereas here we use a binary structure. On the other hand that paper restricts uncertainty to the case in which capital either depreciates fully or partially whereas we treat it as a continuum of shocks. These distinctions is more than aesthetical because his framework allows market shutdowns to occur more easily than here. He uses market shutdowns to explain how learning about true distributions is prevented. Our framework does not allow market shutdowns to occur so easily but is more suitable to explain financial risk capacity.

When modeling the financial sector explicitly, the literature has mostly focused on shocks to the supply and demand of funds provided from the private sector to financial firms. Papers related to Diamond and Dybvig [1983] study coordination games in which depositors may withdraw shortrun funds whereas banks invest long-term. Holmstrom and Tirole [1998] among others study the aggregate provision of funds when the private sector suffers random funding requirements. Both branches of the literature stress the role of bank reserves on efficiency by its effects on incentives from the private sector. Gertler and Karadi [2009] is more related to our paper because they study the effect of the financial systems net-worth on the lending channel. That paper places the stress in the role of limited commitment on the side of banks. Our paper is similar in that the net-worth

\footnotetext{
${ }^{3}$ For example, Gertler and Karadi [2009], Curdia and Woodford [2009], Gertler and Kiyotaki [2010] and Marco Del Negro and Kiyotaki [2010]

${ }^{4}$ Bigio [2010b] provides a broad survey of the literature on liquidity and financial market imperfections
} 
of the financial system determines the amount of liquidity but we place the attention elsewhere: we relate the financial system's net worth to its capacity to bare risk and relate this to asymmetric information.

This paper is valuable from a methodological and quantitative perspective. We don't restrict the analysis around a non-stochastic steady state as is common practice in macro models but rather study the behavior of the model globally and under a broad class of preferences. We are able to do so by using similar aggregation results as in Angeletos [2007] and by following a similar trick to the ones used in the search literature (Lagos and Wright [2005], for example). This prevents us from keeping track of complicated state variables.

\section{The Model}

The model is formulated in discrete time with an infinite horizon. There are two aggregate shocks. A productivity shock $A_{t} \in \mathbb{A}$ where $\mathbb{A} \subset \mathbb{R}$, and a shock $\phi_{t} \in \Phi \subset \mathbb{R}$, to the distribution of capital quality. In particular, $\phi_{t}$, is an index over a family of distributions $f_{\phi}$ of capital quality. The nature of these shocks is explained later. Jointly, $\left(A_{t}, \phi_{t}\right)$ form Markov process that evolves according to a stationary transition probability $\Pi:(\mathbb{A} \times \Phi) \times(\mathbb{A} \times \Phi) \rightarrow[0,1]$. Moreover, $\mathbb{A} \times \Phi$ and $\Pi$ satisfy:

Assumption 1. $\mathbb{A}, \Phi$ are compact. $\Pi$ has the Feller property.

It will be shown that the aggregate state for this economy is given by the aggregate capital stock, $K_{t} \in \mathbb{K}$ in addition to $\left(A_{t}, \phi_{t}\right)$. The aggregate state is summarized by a vector $s_{t}=\left\{A_{t}, \phi_{t}, K_{t}\right\}$, $s_{t} \in \mathbb{S} \equiv \mathbb{A} \times \Phi \times \mathbb{K}$.

\subsection{Demography}

There are two populations with unit measure, entrepreneurs and workers and a set of financial firms. Each entrepreneur is identified by $z \in[0,1]$.

Workers: Workers provide labor elastically and do not save ${ }^{5}$. There instantaneous utility is given by:

$$
U_{t}=\max _{c, l} \frac{1}{\left(1-\frac{1}{\psi}\right)}\left[c-\frac{\varpi}{(1+\nu)}(l)^{1+\nu}\right]^{1-\frac{1}{\psi}}
$$

where $l$ is their labor supply and $c$ there consumption. Workers satisfy a static budget constraint:

$$
c=\omega_{t} l
$$

$\psi$ determines curvature of the workers utility function but plays no role in the model. $\nu$ and $\varpi$ govern the Frisch-elasticity of labor supply and the scale of labor supply respectively. Modifying $\varpi$ is analog to changing the relative population of workers so two population measures are set equal without loss of generality. $\nu>0$ causes aggregate capital to have decreasing returns to scale. Thus, the only role played by workers in this economy is generate decreasing returns to scale in aggregate capital while keeping constant returns to scale at the individual firm level. Workers are not allowed to hold equity but this assumption is innocuous because, since they don't invest, the return to equity would be lower than the return for entrepreneurs.

\footnotetext{
${ }^{5}$ If we allow workers to save, they would not want to do so in equilibrium since the equilibrium return for entrepreneurs would be larger. We come back to this point later on in the paper.
} 
Entrepreneurs: Entrepreneurs do not work. They only invest in equity of other firms and their own which they manage efficiently. Every period, entrepreneurs are randomly assigned one of either type, investors and savers denote by $i$ and $s$ respectively.

The description of preferences here follows from Angeletos [2007] and are of the class introduced by Epstein and Zin [1989]. The preferences of an entrepreneur of type $j$ is given recursively:

$$
V^{j}\left(s_{t}, w_{t}\right)=U\left(c_{t}^{j}\right)+\beta \cdot U\left(\mathcal{C E}_{t}\left(U^{-1}\left(V\left(s_{t+1},, w_{t+1}\right)\right)\right)\right)
$$

where

$$
\mathcal{C} \mathcal{E}_{t}=\Upsilon^{-1}\left(E_{t} \Upsilon(\cdot)\right)
$$

where the expectation is taken over time $t$ information. ${ }^{6}$. The term $\mathcal{C E}_{t}$ refers to the certainty equivalent utility with respect to the CRRA $\Upsilon$ transformation $^{7}$ :

$$
\Upsilon(c)=\frac{c^{1-\gamma}}{1-\gamma} \text { and } U(c)=\frac{c^{1-1 / \sigma}}{1-1 / \sigma}
$$

$\sigma$ measures the intertemporal elasticity of substitution (IES) and $\gamma$ is the coefficient of relative risk aversion (RRA). The expectation is with respect to all realizations of the aggregate state $s_{t}$, the aggregate state, an idiosyncratic $w_{t+1}$ and the entrepreneurs type.

Artificial Financial Firms: Finally, there is a set of financial firms that insure entrepreneurs against risk involved in the purchase of equity. Financial firms are competitive, risk neutral, satisfy a static budget constraint condition. Their objective is to maximize profits statically. The role of these firms is to provide contracts financial contracts in the equity market. Similar artificial introduces by Prescott and Townsend [1984] and Bisin and Gottardi [1999].

\subsection{Production}

There are no informational or enforcement constraints in production ${ }^{8}$. Each entrepreneur runs his firm specific capital endowment, $k_{t} \in[0, \infty]$. Capital is specific to the firm and entrepreneurs increase the capital stock only by undertaking investment projects.

At production, an entrepreneur $z$ takes its firm's capital, $k_{t}(z)$, as given and chooses labor optimally. Production is carried out according to a Cobb-Douglas production function $F(k, l) \equiv$ $k^{\alpha} l^{(1-\alpha)}$. Efficiency requires choosing the labor to capital ratio $l / k$ in order to solve:

$$
\max _{l / k}\left(\left[A_{t} F\left(1, \frac{l}{k}\right)\right]-\omega_{t} \frac{l}{k}\right) k_{t}(z)
$$

where $\omega_{t}$ is the wage payed to the labor factor and $A_{t}$ is the aggregate productivity shock. $k_{t}(z)$ is $z$ 's capital stock. z's capital stock managed evolves according to:

$$
k_{t+1}(z)=i_{t}(z)+\lambda_{t}(z) k_{t}(z)
$$

\footnotetext{
${ }^{6}$ Utility in Epstein and Zin [1989] is defined differently (equation 3.5). This representation is just a monotone transformation of the specification in that paper. By applying $U^{-1}$ to that equation the specification here is obtained.

${ }^{7}$ The transformation $\Upsilon(c)$ characterizes the relative risk aversion through $\gamma>0$. The function $U(c)$ captures intertemporal substitution through $\theta>0$. When $\theta \gamma<1$, the second term in the utility function is convex in $V_{t+1}^{j}$ and concave when the inequality is reverse. When $\gamma=\frac{1}{\theta}$, one obtains the standard expected discounted and equalizing further to 1 yields the log-preference representation.

${ }^{8}$ One can introduce an enforcement problem in production. This would impose a constraint on the entrepreneurs portfolio problem.
} 
where $i_{t}(z)$ is the investment in $z^{\prime}$ s firm. $\lambda_{t}(z)$ is a shock broadly interpreted as combination of a depreciation shock and a permanent and idiosyncratic Solow-neutral productivity shock. This shocks are i.i.d among entrepreneurs but not over time. We refer to $\lambda(z)$ as a depreciation shock but keep the broader interpretation. Hence, $\lambda(z)$ is not restricted to be less than one. Given the aggregate shock $\phi_{t}$,

$$
\lambda_{t}(z) \sim f_{\phi_{t}}
$$

$f_{\phi}$ is random, evolves over time and belongs to a family $\left\{f_{\phi}\right\}_{\phi \in[0,1]}$. At a given period $t$, a member of this family is assigned according to the aggregate shock $\phi_{t} \in[0,1]$. For each $f_{\phi}$, the mean and variance of $\lambda(z)$ are finite and denoted by $\mu_{\phi}$ and $\sigma_{\phi}$. The following assumptions are imposed on the family $\left\{f_{\phi}\right\}_{\phi \in[0,1]}$,

Assumption 2. The family of functions $\left\{f_{\phi}\right\}_{\phi \in[0,1]}$ satisfies the following conditions:

1. $\exists \lambda_{L} \in(0,1)$ such that $F_{\phi}\left(\lambda_{c}\right)=0, \forall \phi$.

2. The function $G_{\phi}\left(\lambda_{c}\right) \equiv \int_{0}^{\lambda_{c}} F_{\phi}(\lambda) d \lambda$ is log-concave, $\forall \phi$.

3. If $\phi_{H}>\phi_{L}$, then $\frac{G_{\phi_{L}}\left(\lambda_{c}\right)}{G_{\phi_{L}}^{\prime}\left(\lambda_{c}\right)} \geq \frac{G_{\phi_{H}}\left(\lambda_{c}\right)}{G_{\phi_{H}}^{\prime}\left(\lambda_{c}\right)}$

The first condition states that for all $\phi$, there is a common lower bound $\lambda_{\phi}$, on $\lambda(z)$. This condition ensures that there is no trivial market breakdown ${ }^{9}$ in the model. Together with second assumption, these conditions guarantee that equilibria are unique. ${ }^{10}$ Finally, the third condition serves the purpose of establishing a monotonic relation between $\phi$ and the degree of market raveling. A family of distributions satisfying the conditions in assumption 2 may be easily constructed. As an example, Figure 1 plots a family Beta distributions that is issued in the simulations in later sections.

\subsection{Entrepreneur Types and Equity Portfolios}

Among the two types of entrepreneurs, only i-entrepreneurs are able to increase their firm's capital stock. Investment opportunities are distributed i.i.d., across time and agents according to a Bernoulli process with probability $\pi$. Hence, depending on this probability, each period, entrepreneurs are segmented into investors and savers with masses $\pi$ and $1-\pi$ respectively. Kurlat [2009] shows that individual shocks to the technical rate of transformation segments entrepreneurs in the same way. ${ }^{11}$

At the beginning of each period, in addition to $k_{t}(z)$, an entrepreneur $z$ owns , $e_{t}^{+}(\tilde{z}, z)$ units of equity originally issued by another other entrepreneur $\tilde{z}$. The counterpart to $e_{t}^{+}(\tilde{z}, z)$ is $e_{t}^{-}(z, \tilde{z})$ which represents $\tilde{z}^{\prime} s$ holdings of equity issued by $z$. A unit of $e_{t}^{+}(z, \tilde{z})$ entitles $z$ to a fraction of the returns generated by $\tilde{z}^{\prime} s$ capital holdings $k_{t}(z)$. For every unit of output generated by $k_{t}(\tilde{z})$,

\footnotetext{
${ }^{9}$ As in Akerlof [1970], when 0 is the lower bound of the support of the lemon quality, there is always an equilibrium with full rationing.

${ }^{10}$ Distribution functions satisfying this condition include the Power (with parameter between 0,1), Weibull, Gamma, Pareto, Log-normal (truncated log-normal for finite support) and Beta (with parameters above 1). Bagnoli and Bergstrom [2005] have a discussion on log-concave families.

${ }^{11}$ Primiceri et al. estimate a model with investment shocks of this forms and argue that they correspond to an important fraction of business cycle volatility. Here, the segmentation is ad-hoc but can be seen as a special case where the technical rates of substitution are 0 and 1 .
} 

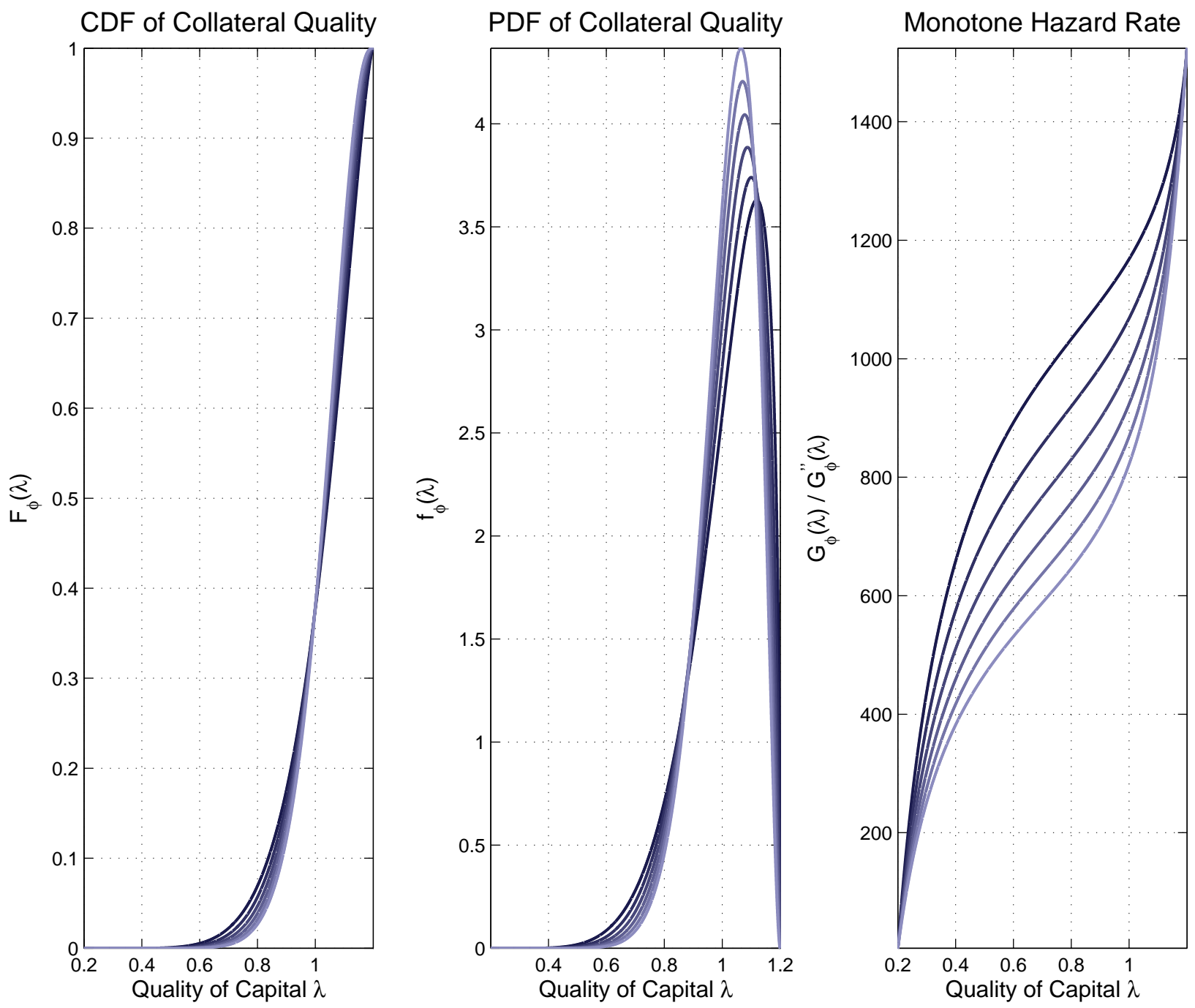

Figure 1: Family of Distributions satisfying conditions in Assumption 2. The figure paints a family of 6 distributions. From left to right, the figures plot the CDF, PDF and the monotone rate for each member. Lighter colors reflect less dispersion. The family corresponds to the baseline calibration in the model. 
$e_{t}^{+}(z, \tilde{z})$ entitles $z$ to the fraction $e_{t}^{+}(z, \tilde{z}) / k_{t}(\tilde{z})$ of these returns. Therefore, equity $e_{t}^{-}(z, \tilde{z})$ is contingent in $\lambda(z)$.

Consistent accounts reiquire

$$
e_{t}^{-}(\tilde{z}, z)=e_{t}^{+}(z, \tilde{z}) \forall z, \tilde{z}
$$

These quantities define functions $e_{t}^{-}(\cdot, z):[0,1] \rightarrow[1, \bar{K}]$ and $e_{t}^{+}(\cdot, z):[0,1] \rightarrow[1, \bar{K}]$ which we restrict to be square measurable $\forall z, t$. The total outside equity for $z$ is its total ownership of other entrepreneurs equity: $\int_{0}^{1} e_{t}^{+}(\tilde{z}, z) d \tilde{z}$. The value of his inside equity is its capital stock minus the total equity it issued $k_{t}-\int_{0}^{1} e_{t}^{-}(\tilde{z}, z) d \tilde{z} \geq 0$. The balance between inside and outside equity defines net equity holdings, $n_{t}(z)$ by,

$$
n_{t}(z) \equiv k_{t}(z)-\int_{0}^{1} e_{t}^{-}(\tilde{z}, z) d \tilde{z}+\int_{0}^{1} e_{t}^{+}(\tilde{z}, z) d \tilde{z}
$$

The following Lemma guarantees that the evolution of $z^{\prime} s$ equity after depreciation shocks are well defined.

Lemma 1 Let $H:[0,1] \rightarrow R$ be a bounded measurable function, and let $e(z) \in L^{2}([0,1])$. Then for a given realization of $s_{t}$, the following holds,

$$
\int H(\lambda(z)) e(z) d z \rightarrow{ }_{p} E\left[H(\lambda) \mid s_{t}\right] \int e(z) d z
$$

Proof. See appendix A for a proof.

Thus, after depreciation, outside and inside equity are respectively,

$$
E\left[\lambda \mid s_{t}\right] \int_{0}^{1} e_{t}^{+}(\tilde{z}, z) d \tilde{z} \text { and } \lambda(z)\left[k_{t}(z)-\int_{0}^{1} e_{t}^{-}(\tilde{z}, z) d \tilde{z}\right]
$$

\subsection{Information, Timing and Equity Markets}

Every period is composed of four stages. Although the timing protocol is such that stages (1-3) occur simultaneously we present a sequential formulation for convenience. We use subscripts $s, t$ to denote variables at stage s of period t. During the first stage, all private information is revealed. During the second stage, entrepreneur sign competitive contracts with financial firms in which they are required to sell equity in exchange for goods. In the third stage, entrepreneurs consume and choose financial composition for their investment projects. Finally, entrepreneurs trade equity once more. Figure 2.4 sketches the timing protocol.

Information Stage: During this stage, aggregate shocks $A_{t}$ and $\phi_{t}$ and the realizations of $\lambda_{t}(\tilde{z})$ for all $\tilde{z}$ are common knowledge. Nevertheless, when entrepreneurs sell equity in the following stage, financial firms are uncapable of distinguishing the original issuer is. Here a buyer cannot distinguish the original issuer of equity while it can distinguish who the seller is. Alternatively, we can assume that only entrepreneurs know the realization of the individual shocks whereas financial firms only know their distribution. In either case, the assumption is meant to capture asymmetric information on the side of a seller. Alternatively, Kurlat [2009] assumes that a firm's capital can be decoupled into several pieces which are affected by different shocks.

Signing Stage: Once information is revealed, financial firms offer contracts in which they commit to provide quantity of goods $x$ in exchange for an amount of equity $e$. After observing the set contracts offered, entrepreneurs decide over the quantity of contracts to sign and a portfolio of equity to give away. Finacial firms will then sell equity during the next stage.

We assume, 


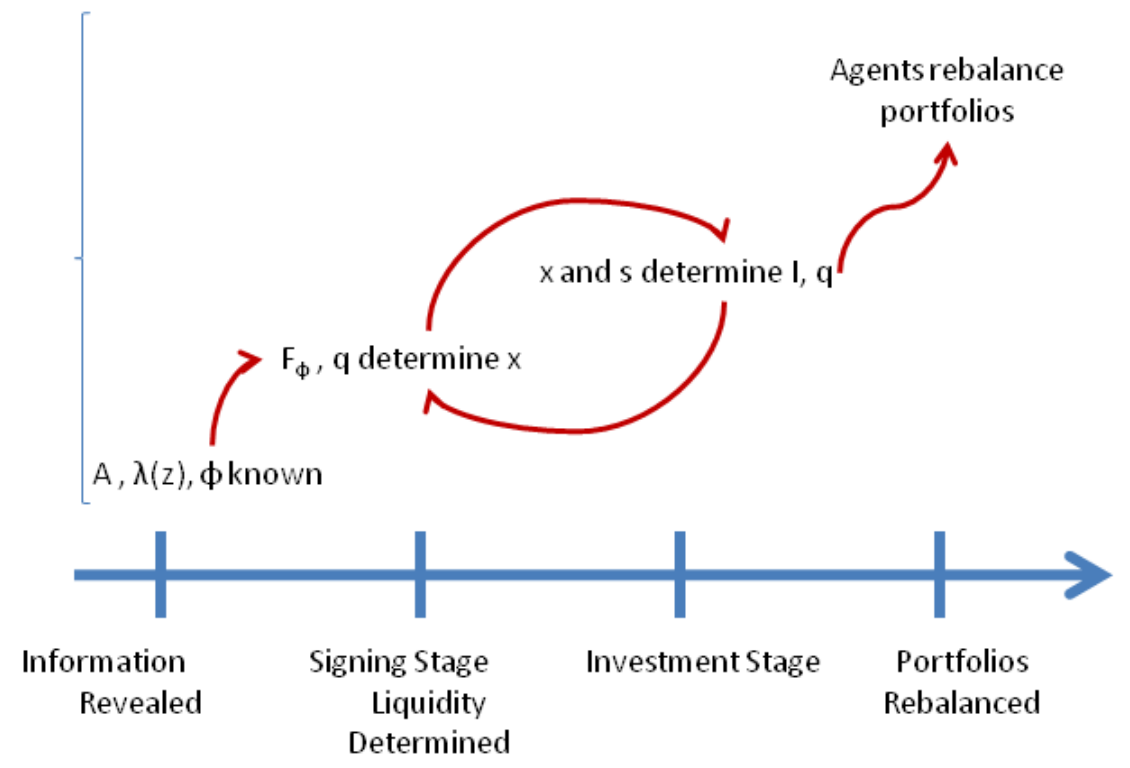

Figure 2: Timing of Events 
Assumption 3. The equity market is anonymous and competitive.

Without this assumption, asymmetric information would not be a problem as separating equilibria would reveal all information. We show this formally in appendix P.

At this stage stage, $z$, sells $\tilde{z}$-equity to financial firms, by an amount $\Delta e_{1, t}^{+}(\tilde{z}, z)$. Since at $(1, t)$, $z$ has $e_{t}^{+}(\tilde{z}, z)$ from the previous period, $e_{1, t}^{+}(\tilde{z}, z) \equiv e_{t}^{+}(\tilde{z}, z)$. In total, $z$ sells $\int_{0}^{1} \Delta e_{1, t}^{+}(\tilde{z}, z) d \tilde{z}$ units of equity and is promised $x_{t}(z)$ at the beginning of the next stage. At the end of the signing stage, its holdings of $\tilde{z}$ equity are $e_{2, t}^{+}(\tilde{z}, z)=\lambda(\tilde{z})\left(\Delta e_{1, t}^{+}(\tilde{z}, z)+e_{1, t}^{+}(\tilde{z}, z)\right)$. On the other hand, its liabilities depreciate so $e_{2, t}^{-}(\tilde{z}, z)=\lambda(z) \int_{0}^{1} e_{t}^{-}(\tilde{z}, z) d \tilde{z}$. Considering all his $(1, t)$ operations, at the beginning of the second stage, $z$ 's balance sheet is:

$$
\begin{aligned}
n_{2, t}(z) & =\lambda(z)\left(k_{t}(z)-\int_{0}^{1} e_{t}^{-}(\tilde{z}, z) d \tilde{z}\right)+\int_{0}^{1} \lambda(\tilde{z})\left(\Delta e_{1, t}^{+}(\tilde{z}, z)+e_{1, t}^{+}(\tilde{z}, z)\right) d \tilde{z}+x_{t}(z) \\
& =\lambda(z) k_{t}(z)-\int_{0}^{1} e_{2, t}^{-}(\tilde{z}, z) d \tilde{z}+\int_{0}^{1} e_{2, t}^{+}(\tilde{z}, z) d \tilde{z}+x_{t}(z)
\end{aligned}
$$

On the other side of the market, financial firms owe $\int_{0}^{1} x_{t}(z) d z$ to i-entrepreneurs and hold an amount of equity,

$$
-\int_{0}^{1} \int_{0}^{1} \lambda(\tilde{z}) \Delta e_{1, t}^{+}(\tilde{z}, z) d \tilde{z} d z
$$

In equilibrium, competition among financial firms requires a zero profit contidion so heir asetts and liabilities will be equated.

Investing Stage: At the third stage financial firms sell all their equity holdings 3 at a price that internalizes $\lambda(\tilde{z})$. Given that shocks are i.i.d, once equity is depreciated correspondingly it becomes a homogeneous asett. Therefore, the financial sector is to deal with private information. Financial firms internalize the price realized at this stage when they offer contracts in the sigining stage. Entrepreneurs decide on consumption, investment and the financing structure of investment projects.

Investing entrepreneurs: An i-entrepreneurs $z$ 's budget constraint is:

$$
c_{t}+i_{t}+q_{t} \int_{0}^{1} \Delta e_{2, t}^{+}(\tilde{z}, z) d \tilde{z}=r_{t} n_{t}(z)+x_{t}(z)+q_{t} i_{t}^{s}
$$

This budget is written in real terms. $q_{t}$ is the price of equity in terms of consumption goods. The right hand side corresponds to the entrepreneur's funds. The first term is the return to equity holding from the previous period where $r_{t}$ is the per unit of equity return. The second term is $x_{t}(z)$ which corresponds to the amount delivered by financial firms. Finally, $q_{t} i_{t}^{s}$, are resources obtained by new issues of equit $i_{t}^{s}$. Funds are used to consume $c_{t}$, to finance new projects at scale $i_{t}$, and purchase equity in amount $\int_{0}^{1} \Delta e_{2, t}^{+}(\tilde{z}, z) d \tilde{z}$. At this stage, the entrepreneur holds equity but he is not allowed to sell it. We don't allow trade to reopen becuase, stages 2 and 3 occur simultaneously although we present them sequentially.

When the i-entrepreneur chooses a scale for investment $i_{t}$ it is also choosing a financing structure. Each project is funded by internal funds $i_{t}^{d}$ and external funds $i^{f}$ obtained by issuing equity so $i_{t}=i_{t}^{d}+i_{t}^{f}$. The price of equity is $q_{t}$ so if $i_{t}^{s}$ units are issued, external financing is $i^{f}=q_{t} i_{t}^{s}$. Note that after investment is realized, $i$-entrepreneurs increase own $i_{t}^{i}=i_{t}-i_{t}^{s}$ while they provided $i_{t}^{d}=i_{t}-q_{t} i_{t}^{s}$. Thus, when, $q_{t}>1$ the composition of the ownership and funding differ. We can use this By substitution, we can express the i-entrepreneurs budget constraint as: 


$$
c_{t}+i_{t}^{d}+q_{t} \int_{0}^{1} \Delta e_{2, t}^{+}(\tilde{z}, z) d \tilde{z}=r_{t} n_{t}(z)+x_{t}(z)
$$

$i$-entrepreneurs: face a restriction to external financing because of limited commitment at the time of investment. In particular, they can default on their new equity issues and keep a fraction $1-\theta$ of the investment project. Since there are no enforcement technologies available, the incentive compatibility condition is

$$
(1-\theta) i_{t} \leq i_{t}^{i} \text {, or equivalently, } i_{t}^{s} \leq \theta i_{t}
$$

This condition states that the lenders stake in the project may not exceed $\theta$. The inverse of this parameter determines the marginal leverage ratio for investment. Note that here, only investment suffers from limited commitment whereas there are no restriction on equity backed by existing capital. This assumption is natural: equity backed by investment is after all, only a promise to create capital in the future. Once capital is in place, equity can be fully pledged using capital as collateral. ${ }^{12}$

s-entrepreneurs: An s-entrepreneur $z$ faces a budget constraint:

$$
c_{t}+q_{t} \int_{0}^{1} \Delta e_{2, t}^{+}(\tilde{z}, z) d \tilde{z}=r_{t} n_{t}
$$

This budget is an analog to the i-entrepreneur's budget where the only distinction is that the possibility to invest is not available so $i_{t}^{s}(z)=0$. Agents only participate in the equity market by purchasing assets from financial firms. Outside equity evolves according to $e_{3, t}^{+}(\tilde{z}, z)=\Delta e_{2, t}^{+}(\tilde{z}, z)+$ $e_{2, t}^{+}(\tilde{z}, z)$ and total issued equity follows,

$$
\int_{0}^{1} e_{t+1}^{-}(\tilde{z}, z)=i_{t}^{s}(z)+\lambda(z) \int_{0}^{1} e_{t}^{-}(\tilde{z}, z)
$$

Clean up stage: At the final stage, agents are allowed to trade once more. We introduce this stage as a technical step that prevents equity portfolios from becoming state variables. At this stage, portfolio holdings of i-entrepreneurs are concentrated in their own firms equity. Therefore, i-entrepreneurs face some idiosyncratic risk. Agents choose to sell and buy equity in order to recompose their portfolios. Therefore, $z$ will choose $\Delta e_{t}^{+}(\tilde{z}, z), \forall \tilde{z}$ such that:

$$
q_{t}\left[\int_{0}^{1} \Delta e_{3, t}^{+}(\tilde{z}, z)+\Delta e_{3, t}^{-}(\tilde{z}, z) d \tilde{z}\right]=0
$$

New new issues of equity are limited by the amount of inside equity,

$$
\int_{0}^{1} \Delta e_{3, t}^{+}(\tilde{z}, z) \leq k_{t}(z)-\int_{0}^{1} e_{3, t}^{-}(\tilde{z}, z)
$$

Inside and outside equity evolve according to $e_{t+1}^{+}(\tilde{z}, z)=\Delta e_{3, t}^{+}(\tilde{z}, z)+e_{3, t}^{+}(\tilde{z}, z)$ and $e_{t+1}^{-}(\tilde{z}, z)=$ $\Delta e_{3, t}^{-}(\tilde{z}, z)+e_{3, t}^{-}(\tilde{z}, z)$.

\footnotetext{
${ }^{12}$ One can alternatively assume that the entrepreneur can default on all his equity. Since investment opportunities are random in our environment, one would need to keep track of individual liabilities and net equity to find the individual incentive compatibility constraints. To avoid this issue, one could assume investment opportunities are alway available but would need to introduce a new agent in the economy and prevent entrepreneurs to grow away from the constraints.
} 


\subsection{Collapsing Stages 1,2 and 3}

The three stage environment of the model can be collapsed into a single state problem where entrepreneurs choose consumption and equity. In this section we show once we solve for the signing equilibrium, by substituting the optimal policies at stages 2 and 4 , and the optimal financing problem at stage 3 , the problem from the perspective of stage 3 becomes standard. We follow these steps using backward induction. As a first step, we can show that if the value function is a a function a definition of wealth that depends on portfolio compositions we can assert,

Proposition 1 (Net Equity) The value function at stage 3 is an increasing function of net equity only.

This result is shown formally in appendix B. The proof follows directly from the fact that agents will trade in order to diversify risk as much as possible. Since trades are constrained by the level of equity, portfolio weights will be identical for all entrepreneurs and scaled by their equity. This result is stated formally in Proposition 4 also in appendix B. Hence, at stage 3, entrepreneurs don't care about the composition of their equity holdings but only about the total amount of equity they hold.

We use this proposition 1, to derive the optimal financial decisions of i-entrepreneurs during the third stage.

Proposition 2 (Stage 2 Characterization) If $q_{t}>1$, the solution to Problem 5 is

$$
\Delta e_{2, t}^{+}(\tilde{z}, z)=0 \text { and } i_{t}^{s}=\theta i_{t}
$$

Observe that when $q>1$, an investment project $i_{t}$, an i-entrepreneur finances $i_{t}^{d}=i_{t}-q_{t} i_{t}^{s}=$ $i_{t}\left(1-\theta q_{t}\right)$. Because the constraint binds, the entrepreneur owns $(1-\theta) i_{t}$. Thus, when investing, i-entrepreneurs place a downpayment $\left(1-\theta q_{t}\right)$ consumption units and to obtain $(1-\theta)$ units of equity. Becuase the budget is written in real terms, this defines a cost of generating equity for the investing entrepreneur,

$$
q_{t}^{R}=\left[\frac{\left(1-\theta q_{t}\right)}{(1-\theta)}\right] \leq 1 \text { with equality if } q_{t}=1
$$

It is shown that in equilibrium, $q_{t} \in\left[1, \frac{1}{\theta}\right)$, so this quantity is well defined. The difference between $q_{t}$ and $q_{t}^{R}$ is a wedge between the cost of purchasing outside equity and the cost of generating inside equity.

From the perspective of stage 3 , entrepreneurs choose $i_{t}^{d}$ and $c_{t}$ subject to the constraint given by (5). The quantities $x_{t}$ and $\Delta e_{1, t}^{+}(\tilde{z}, z)$ are determined determined in stage 2 . For this, we study a competitive equilibrium in the spirit of Prescott and Townsend [1984] and Bisin and Gottardi [1999]. A contract $c$ is a pair $\left(x_{c}, e_{c}\right) \in R_{+}^{2}$ that represent an amount $x_{c}$ of goods promised to be delivered to the entrepreneur in exchange for $e_{c}$ units of equity. A symmetric competitive signing equilibrium is defined as,

Definition (Symmetric Competitive Signing Equilibrium). A symmetric competitive signing equilibrium is a set of offered contracts $C$, a portfolio of equity $\Delta e_{t+1}^{+}(\tilde{z})$ and a measure $\mu_{c}$ on the set of of contracts such that:

1. All offered contracs $c \in C$ are profitable for financial firms given the portfolio:

$$
x_{c}-q_{t} \int_{0}^{1} \lambda(\tilde{z}) \Delta e_{t+1}^{+}(\tilde{z}) d \tilde{z} \geq 0
$$


2. The measure and portfolio maximize the entrepreneurs wealth,

$$
\max _{\mu_{c} \in M(C), \Delta e_{t+1}^{+}(\tilde{z})} \int_{C} x_{c}-q_{t}^{R}\left(\int_{0}^{1} \lambda(\tilde{z}) \Delta e_{t+1}^{+}(\tilde{z}) d \tilde{z}\right) \mu_{c}(d c)
$$

3. And the following feasibility constraint is satisfied:

$$
e_{t+1}^{+}(\tilde{z}, z) d \tilde{z}+n(z) \int_{C} \Delta e_{t+1}^{+}(\tilde{z}) \mu_{c}(d c) \geq 0, \forall \tilde{z}
$$

The first condition requires that financial firms make no losses when offering contracts. The second condition implies that entrepreneurs choose the most convenient contracts amongst those offered and the final condition requires them not to issue equity entitled to other entrepreneurs.

Proposition 3 shows that competitive equilibria are characterized by a unique pooling contract. Equilibrium is presented at this degree of generality because we don't desire to a particular market structure. Thus, pooling in this environment is an equilibrium outcome and not imposed. The equilibrium is characterized by,

Proposition 3 (Signed Contracts) Given prices $\left(q, q^{R}\right)$, every a Competitive Symmetric Signing Equilibrium is characterized by the unique pooling contract $\left(x_{*}, \lambda_{*}\right)$ that solves

$$
\lambda_{*}=\min \left\{1, \frac{x_{*}}{q_{t}^{R}}\right\}
$$

and

$$
x_{*}=q_{t} E_{\phi}\left[\lambda(\tilde{z}) \mid \lambda(\tilde{z}) \leq \lambda_{c}\right] \text { if } q>1 \text { and } x_{*}=0 \text { otherwise }
$$

where $e_{*}=F\left(\lambda_{*}\right)$ and $\mu\left(x_{*}, \lambda_{*}\right)=1$.

Moreover, the agents optimal policy imply that,

$$
\begin{gathered}
x_{t}=x_{*} F_{\phi}\left(\lambda_{c}\right) n_{t}(z) \\
\Delta e_{t+1}^{+}(\tilde{z}, z)=-e_{t+1}^{+}(\tilde{z}, z) \text { if } \lambda(\tilde{z}) \leq \lambda_{*} \text { and } \\
\Delta e_{t+1}^{+}(\tilde{z}, z)=0 \text { otherwise }
\end{gathered}
$$

Under assumption $2.3\left(x_{*}, \lambda_{*}\right)$ are increasing in $\phi$.

A detailed derivation of this characterization is provided in appendix D. This proposition states that for every pair $\left(q, q^{R}\right)$, a solution to a competitive contract is obtained by solving a the pair of equations given by (9) and (10). In the standard lemons market, multiple solutions may solve this pair of equations (Wilson [1979] and Wilson [1980]). Rather than refining the notion of equilibria, we chose to impose assumption 2 which restricts attention to a set of the density families $f_{\phi}$ such that equilibria a uniqueness result holds.

Let's recap what we've learned so far. By Proposition 4, agents will enter the first stage with a homogeneous portfolio. Given a price $q$, Proposition 3 shows how is $x_{t}$ determined. $x_{t}$ is used to consume or finance the downpayment in stage 3. Proposition 2 shows that i-entrepreneurs won't buy equity during stage 3 and again by Proposition 4, are not concerned about portfolio compositions. Thus, the whole focus can be put on the desission from stage two. We combine the results from Propositions 3 and 2 and the budget constraints (4) and (2) and the evolution of equity to recast the entrepreneurs' budget constraints in a concise way. We formalize the result by, 
Proposition 4 (Entrepreneurs Wealth) Wlog, the entrepreneurs budget constraint can be written as

$$
\begin{aligned}
c_{t}+q_{t}^{R} n_{t+1}(z) & =\left(r_{t}+q_{t}^{i} E_{\phi}[\lambda]\right) n(z) \equiv w_{t}^{i} \\
c_{t}+q_{t} n_{t+1}(z) & =\left(r_{t}+q_{t} E_{\phi}[\lambda]\right) n(z) \equiv w_{t}^{s}
\end{aligned}
$$

correspondingly for investing and saving entrepreneurs. $q_{t}^{i}$ is given by

$$
q_{t}^{i}=q_{t} \frac{E_{\phi}\left[\lambda(\tilde{z}) \mid \lambda(\tilde{z}) \leq \lambda_{c}\right]}{E_{\phi}[\lambda(\tilde{z})]} F_{\phi}\left(\lambda_{c}\right)+q_{t}^{R}\left(1-F_{\phi}\left(\lambda_{c}\right)\right) \frac{E_{\phi}\left[\lambda(\tilde{z}) \mid \lambda(\tilde{z}) \geq \lambda_{c}\right]}{E_{\phi}[\lambda(\tilde{z})]}
$$

Proof. See appendix E.

$w_{t}^{i}$ and $w_{t}^{s}$ represent the entrepreneurs liquidity adjusted wealth. $q_{t}^{i}$ defined in (11) is the value of a unit of the investing entrepreneur's portfolio. The interpretation of this price is simple. The fraction $F_{\phi}\left(\lambda_{c}\right)$ of its equity holdings is transferred to financial firms. The average depreciation among this portfolio is $E_{\phi}\left[\lambda(\tilde{z}) \mid \lambda(\tilde{z}) \leq \lambda_{c}\right]$. The entrepreneur values each unit of this portfolio at $q_{t}$ because it is the effective price it received by contract. The remaining fraction $\left(1-F_{\phi}\left(\lambda_{c}\right)\right)$ of equity, has an average depreciation $E_{\phi}\left[\lambda(\tilde{z}) \mid \lambda(\tilde{z}) \geq \lambda_{c}\right]$. Since this equity is kept, it must be valued at the same price at the cost of increasing equity $q_{t}^{R}$. In fact, the entrepreneur could have chosen to sell this equity and use it for a downpayment. Since, $q_{t}^{R}=1$ when $q_{t}=1$ so $q_{t}^{i}=1$. $q_{t}=1$, equity is not traded and both entrepreneurs are equal. Thus, this budget constraints hold in all states. It is useful to define two additional quantities related to the entrepreneurs wealth:

$$
\varphi_{t}=E_{\phi}\left[\lambda(\tilde{z}) \mid \lambda(\tilde{z}) \leq \lambda_{c}\right] F_{\phi}\left(\lambda_{c}\right)
$$

$\varphi_{t}$ represents the fraction of equity transferred to financial firms after depreciation. This amount can be interpreted as a measure of liquidity. $\psi_{t}$, is the counterpart to $\varphi_{t}$,

$$
\psi_{t} \equiv\left(1-F\left(\lambda_{c}\left(s_{t}\right)\right)\right) E_{\phi}\left[\lambda(\tilde{z}) \mid \lambda(z)>\lambda_{c}\right]
$$

By definition, this functions satisfy, $\varphi_{t}+\psi_{t}=E_{\phi}[\lambda]$. In $\mathrm{KM} \varphi_{t}$ and $\psi_{t}$ are exogenous and random. In Kurlat [2009], the amount of liquidity is also obtained similarly. We return to this quantities when we study the equilibrium in the following section.

Proposition 4 allows us to forget about the interim stages. For each type, the relevant state variable for the entrepreneur is a single state variable, $n_{t}$. Given $s_{t}$, there is a contract that determines the price $q_{t}^{i}$ and $q_{t}$. Saving and i-entrepreneurs will then choose investment in inside and outside equity. Thus, from the perspective of stage 2 , the entrepreneur will have optimally signed a contract that provides him with funds it can use and knows that it will fully diversify its portfolio in the final stage. We get rid of the time subscripts and describe the agents problem recursively.

Problem 1 (saver's problem)

$$
V^{s}\left(w^{s}, s\right)=\max _{c^{s}, n^{\prime}} U\left(c^{s}\right)+\beta \cdot U\left(\mathcal{C E}\left(U^{-1}\left(V^{j}\left(w^{\prime}, s^{\prime}\right)\right)\right)\right)
$$

subject to

$$
c^{s}+q(s) n^{\prime}=w^{s}
$$

Here the superscrit $\mathrm{j}$ in $V^{j}$ refers to the agents type next period. The investing entrepreneur solves a similar problem: 
Problem 2 (investor's problem)

$$
V^{i}\left(w^{i}, s\right)=\max _{c^{i}, n^{\prime}} U\left(c^{i}\right)+\beta \cdot U\left(\mathcal{C} \mathcal{E}\left(U^{-1}\left(V\left(w^{\prime}, s^{\prime}\right)\right)\right)\right)
$$

subject to

$$
c^{i}+q^{R}(s) n^{\prime}=w^{i}
$$

Note that when choosing $n^{\prime}$, the investing entrepreneur is implicitly choosing $i^{d}$. The inequality in the definition follows from $i^{i} \leq(1-\theta) i$. Note also that $n$ is not a state variable because it can be recovered from $(w, s)$. Finally, workers provide labor $l_{t}$ to production in exchange for consumption goods $c_{t}$ in order to maximize their static utility. We end this section with an important remark about the market structure in this model. In particular, we could have got rid of all equity trade in the model an study a model in which capital can be disentagled into pieces that suffered different shocks. $^{13}$

Remark 1. The model is isomorphic to as setup where capital within a firm is perfectly separable and whose pieces are depreciated according to i.i.d $\lambda$ shocks.

\section{Equilibrium}

Here we define our notion of competitive equilibrium. The definition exploits the results of the previous section to focus on the reduced form versions of the agents problems. This reduction is convenient as we only need to keep track of the evolution of aggregate equity without carrying distributions of portfolios in the model. On the other hand, the only requirement imposed in the definition is that the amount of liquid assets $x_{t}$ be given by with the distribution $f_{\phi}$ and $q_{t}$ following Proposition 20. We avoid the time time subscripts since we study the recursive version of the model.

Definition (Recursive Competitive Equilibrium). A recursive competitive equilibrium (symmetric) is a set price functions, $q(s): \mathbb{S} \rightarrow \mathbb{R}_{+}, \omega(s): \mathbb{S} \rightarrow \mathbb{R}_{+}$, and a liquid asset function $x(s): \mathbb{S} \rightarrow \mathbb{R}_{+}$, allocations $n^{\prime, j}, c^{j}, l^{j}, i^{j}$, for $j=i, s, w$, and a sequence of wealth distributions $\Lambda_{t}$, and an invariant transition function $\Xi: \mathbb{S} \times \mathbb{S} \rightarrow[0,1]$ for the aggregate state $s$ such that the following conditions are met,

1. $x(s)$ solves is the unique solution to (9) and (10) given $q(s)$.

2. Optimality of Policies: $c^{j}(s), n^{j, \prime}(s), i^{j, \prime}(s), i^{j, \prime}(s), j=i, s, w$, solve the problems of entrepreneurs and workers.

3. Goods, labor and equity markets clear.

4. Capital evolves according to $K \prime(s)=I(s)+\lambda K(s)$

5. The distribution $\Lambda_{t}$ and the transition functions $\Xi$ are consistent with the policy functions.

Optimal Policies: The problems of both entrepreneurs are similar except for their budget constraints. Entrepreneurs choose consumption and equity holdings next period which differ in the cost of equity. The following propositions describes the policy functions as linear functions of wealth. This result is similar to the one obtained by Angeletos [2007] and allows to define a competitive recursive equilibrium without the need of tracking the evolution of wealth distributions. Thus, thanks to this result, the definition is internally consistent. The main issue here is dealing with the liquidity constraints.

\footnotetext{
${ }^{13}$ This relates to the setup in Kurlat [2009].
} 
Proposition 5 (Savers policies) For s-entrepreneurs are given by the following:

$$
\begin{aligned}
c^{s} & =\left(1-\varsigma^{s}(s)\right) w^{s} \\
q(s) n^{\prime} & =\varsigma^{s}(s) w^{s}
\end{aligned}
$$

For i-entrepreneurs, their optimal policies are characterized by,

Proposition 6 (investors policies) Optimal allocations for $i$-entrepreneurs are given by the following:

(i) For all $s$ such that $q(s)>1$, optimal policies are given by:

$$
\begin{aligned}
c^{i} & =\left(1-\varsigma^{i}(s)\right) w^{i} \\
q^{R}(s) n^{\prime} & =\varsigma^{i}(s)\left(r(s)+\lambda q^{i}(s)\right) n=\varsigma^{i}(s) w^{i} \\
i & =\frac{n^{\prime}-\psi(s) n}{(1-\theta)}
\end{aligned}
$$

(ii) For all $s_{t}$ such that $q_{t}=1$, policies are identical to the s-entrepreneurs and $i_{t}$ is indeterminate at the individual level.

Propositions 5 and 6 show an important property of the model. As long as we know the marginal propensities to save of both types, $\varsigma^{s}(s)$ and $\varsigma^{i}(s)$, we can compute the equilibrium without the need to keep track of individual state variables. Before we characterize $\varsigma^{s}(s)$ and $\varsigma^{i}(s)$ we need to define conditional returns:

$$
\begin{aligned}
R^{s s}\left(s, s^{\prime}\right) & \equiv \frac{r\left(s^{\prime}\right)+q\left(s^{\prime}\right) E_{\phi}[\lambda]}{q(s)} \text { and } R^{i s}\left(s, s^{\prime}\right) \equiv \frac{r\left(s^{\prime}\right)+q\left(s^{\prime}\right) E_{\phi}[\lambda]}{q^{R}(s)} \\
R^{i i}\left(s, s^{\prime}\right) & \equiv \frac{r\left(s^{\prime}\right)+q^{i}\left(s^{\prime}\right) E_{\phi}[\lambda]}{q^{R}(s)} \text { and } R^{i s}\left(s, s^{\prime}\right) \equiv \frac{r\left(s^{\prime}\right)+q^{i}\left(s^{\prime}\right) E_{\phi}[\lambda]}{q(s)}
\end{aligned}
$$

$R^{j m}\left(s, s^{\prime}\right)$ is the s' contingent return of equity from to an entrepreneur $\mathrm{m}$ who becomes $\mathrm{j}$ as at the current state is s. $R^{s}\left(s, s^{\prime}\right)=(1-\pi) R^{s s}\left(s, s^{\prime}\right)+\pi R^{i s}\left(s, s^{\prime}\right)$ and and $R^{i}\left(s, s^{\prime}\right)=(1-\pi) R^{i s}\left(s, s^{\prime}\right)+$ $\pi R^{i i}\left(s, s^{\prime}\right)$. This returns are use to obtain the marginal propensities to save, $\varsigma^{i}(s)$ and $\varsigma^{s}(s)$ :

Proposition 7 (Recursion) The functions $\varsigma^{i}(s)$ and $\varsigma^{s}(s)$, in propositions 5 and 6 solve the following system of difference equations:

$$
\begin{aligned}
& \left(1-\varsigma^{i}(s)\right)^{-1}=1+\beta^{\sigma} \Omega^{i}\left(\left(1-\varsigma^{i}\left(s^{\prime}\right)\right)^{\frac{1}{1-\sigma}},\left(1-\varsigma^{s}\left(s^{\prime}\right)\right)^{\frac{1}{1-\sigma}}\right)^{\sigma-1} \\
& \left(1-\varsigma^{s}(s)\right)^{-1}=1+\beta^{\sigma} \Omega^{s}\left(\left(1-\varsigma^{i}\left(s^{\prime}\right)\right)^{\frac{1}{1-\sigma}},\left(1-\varsigma^{s}\left(s^{\prime}\right)\right)^{\frac{1}{1-\sigma}}\right)^{\sigma-1}
\end{aligned}
$$

and

$$
\Omega^{i}\left(a^{s}, a^{i}\right)=\Upsilon^{-1} \mathrm{E}\left[(1-\pi) \Upsilon\left(a^{s}\left(s^{\prime}\right) R^{s s}\left(s^{\prime}\right)\right)+\pi \Upsilon\left(a^{i}\left(s^{\prime}\right) R^{s i}\left(s^{\prime}\right)\right)\right]
$$

and

$$
\Omega^{i}\left(a^{s}, a^{i}\right)=\Upsilon^{-1} \mathrm{E}\left[(1-\pi) \Upsilon\left(a^{s}\left(s^{\prime}\right) R^{i s}\left(s^{\prime}\right)\right)+\pi \Upsilon\left(a^{i}\left(s^{\prime}\right) R^{i i}\left(s^{\prime}\right)\right)\right]
$$

In addition, $\varsigma_{t}^{i}, \varsigma_{t}^{i} \in(0,1)$, are defined uniquely and satisfy

$$
\begin{aligned}
& \varsigma^{i}>\varsigma^{s} \text { whenever } \sigma>1 \text { and } \\
& \varsigma^{i}<\varsigma^{s} \text { whenever } \sigma \in(0,1)
\end{aligned}
$$


The proof of Propositions 5, 6 and 7 is proved jointly in appendix $\mathrm{H}$ and is similar to a proof in Angeletos [2007]. In essence, Proposition 7 shows that marginal propensities to save of both types solve a non-linear functional equation which can be easily solved by repeated iteration. This result allows us to establish as a corollary that the economy admits aggregation. Also, note that the when $1 / \sigma=\gamma=1$, the economy becomes identical to KM when liquidity shocks arise endogenously (and without additional assets). One can guess and verify that $\varsigma^{s}=\varsigma^{i}=\beta$ solve (14) and (15). As a final by-product, we obtain closed form solutions for welfare function which are useful for policy analysis. The rest of the equilibrium conditions determining the evolution of aggregate variables is relegated to appendix I. The next section characterizes some of the features of the equilibrium.

\subsection{Characterization}

Equity market clearing: In equilibrium the price $q$ is bounded.

Lemma 2 In equilibrium $q \in\left[1, \frac{1}{\theta}\right]$.

Proof. See appendix F.

$q$ never falls below one by arbitrage as no agent would invest and would only buy equity from others. This is true also because capital is reversible. Similar models with irreversibility do not have this property ${ }^{14}$. The model can be easily adapted to allow for this situation. In turn, q can never be above $\frac{1}{\theta}$ because this would violate the condition (6). In equilibrium, investment satisfies,

Corollary 1 Investment is Pareto efficient (first best) iff $q=1$. If $q>1$, investment it is constrained-Pareto inefficient and below first best.

Proof. See appendix G.

We use Proposition 2 to characterize the rest of the equilibrium. It is useful to understand the equilibrium by its pieces. The quantities $\varphi$ and $\psi$ of liquid and illiquid assets are functions of $q$ and the distribution of capital quality $f_{\phi}$ as described by Proposition 3. In turn, $\varphi$ and $\psi$ define the amount of liquid funds $x$ which are used as collateral. The marginal propensities to save $\varsigma^{s}$ and $\varsigma^{i}$ describe the demand for new investment projects. When liquidity is innsuficient, $q$ will adequate reducing the demand for new investment projects of s-entrepreneurs and increasing the downpayment of i-entrepreneurs as their value arbitrage grows. $q$ accomodates up to the point wher the incentive compatibility constraint (6). With investment determined, future returns are determined and thus so are $\varsigma^{s}, \varsigma^{i}$. Thus, to compute and equilibrium we look for a fixed point for the functions $\left(\varphi, \psi, q, \varsigma^{s}, \varsigma^{i}\right)$.

To make further progress we begin analyzing aggregate investment taking $\varsigma^{s}, \varsigma^{i}, \varphi$ and $\psi$ and the aggregate state $s$ as given. Using $\psi$, the balance sheet of the i-entrepreneur reads

$$
n^{\prime}(z)=i^{i}+\psi(s) n(z)
$$

Aggregating this balance sheets with respect to $z$ we obtain the aggregate supply of investment projects (recall 3).

\footnotetext{
${ }^{14}$ In Sargent [1980], irreversible investment decision cause $\mathrm{q}$ to fall below one. This happens when if at $\mathrm{q}=1$ the demand for consumption goods generates negative investment. Q must adjust to fall below one such that the demand for investment is 0 .
} 


$$
I^{i}(q)=\pi\left[\int n^{\prime, i}(w) \Lambda(d w)-\psi(s) K\right]
$$

$I_{t}^{i}$ is the fraction for aggregate investment owned by i-entrepreneurs. On the other hand, $I_{t}^{s}$ is the demand for investment by s-entrepreneurs and is given similarly by the s-entrepreneurs' balance sheet. Since financial firms supply the equity they bought from i-entrepreneurs in the first stage, the demand for investment equals is implicitly given by the demand for equity created this period:

$$
\underbrace{(1-\pi)\left(\int n^{\prime, s}(w) \Lambda_{t}(d w)-E_{\phi}[\lambda(\phi)] K\right)}_{\text {Equity Demand S-types }}=\underbrace{\pi \varphi(s) K}_{\text {Equity Supply - Fin. Firms }}+\underbrace{I^{s}(q)}_{\text {Demand - Investment }}
$$

The aggregate version of incentive compatibility constraint (6) is,

$$
I^{i}(q) \leq(1-\theta)\left(I^{i}(q)+I^{s}(q)\right)
$$

When $q>1$, Lemma 6 ensures that the constraint must hold with equality. In equilibrium, taking the optimal policies from Propositions 5 and 6 as given, $I^{i}$ and $I^{s}$ are continuous functions of $q$. In fact, $I^{i}$ is increasing and $I^{s}$ decreasing in $q$. If $I^{i}(1)$ and $I^{s}(1)$ satisfy (18), then $q_{t}=1$, and aggregate investment is $I_{t}=I_{t}^{i}(1)+I_{t}^{s}(1)$. Otherwise, $q_{t}$ is the value of $q$ that solves (18) with equality. Thus we have shown,

\section{Remark 2.}

$$
\begin{aligned}
q= & \max \left(1, q^{*}\right) \\
& \text { where } q^{*} \text { is the smallest } q \text { solving } \\
I^{i}(q)= & (1-\theta)\left(I^{i}(q)+I^{s}(q)\right)
\end{aligned}
$$

and aggregate investment is defined by,

$$
I=I^{i}(q)+I^{s}(q)
$$

As a function of $\left(\varphi, \psi, \varsigma^{s}, \varsigma^{i}\right), q_{t}$ can be computed analytically according to the following proposition,

Proposition 8 (Market Clearing) Taking the liquidity functions $\varphi$ and $\psi$ and the marginal propensities to save $\varsigma^{s}$ and $\varsigma^{i}$ as given, there exists a unique $q$ that clears out the equity market and it is given by:

$$
q=\left\{\begin{array}{c}
1 \text { if } 1>q_{2}>q_{1} \\
q_{2} \text { if } q_{2}>1>q_{1} \\
1 \text { if otherwise }
\end{array}\right.
$$

where the terms $\left(q_{1}, q_{2}\right)$ are positive and given by:

$$
\begin{aligned}
& q_{1}=\frac{B^{2}}{2 A}-\frac{\sqrt{B^{2}-4 A C}}{2 A} \\
& q_{2}=\frac{B^{2}}{2 A}+\frac{\sqrt{B^{2}-4 A C}}{2 A}
\end{aligned}
$$


where

$$
\begin{aligned}
& A=-\theta\left((1-\pi)\left(1-\varsigma^{s}\right) E_{\phi}[\lambda]+\pi\left(1-\varsigma^{i}\right) \varphi-\theta \frac{\left(1-\varsigma^{i}\right) \psi}{(1-\theta)} \pi\right) \\
& B=\theta r\left((1-\pi) \varsigma^{s}+\pi \varsigma^{i}\right)+\left((1-\pi)\left(1-\varsigma^{s}\right) E_{\phi}[\lambda]+\pi \varphi\right)-\theta \frac{\left(1-\varsigma^{i}\right) \psi}{(1-\theta)} \pi \\
& C=-(1-\pi) \varsigma^{s} r
\end{aligned}
$$

Proof. See appendix J.

Proposition 8 describes $q$ as a function of the agents policies $\varsigma^{s}$ and $\varsigma^{i}$ and the liquidity variables $\varphi$ and $\psi . q$ defines via Proposition 3 the threshold $\lambda_{*}$ that pins down $\varphi$ and $\psi$. The optimal marginal propensities derived from 7 depend on $q$ and recursively on themselves and future returns. Future returns depend on $(K, A)$ which evolve according to the transition probability $\Lambda . \Lambda$ depends on aggregate investment, $I$. This conditions define an explicity mapping from the space of $q, \varphi, \psi, \varsigma^{s}, \varsigma^{i}$. This mapping is well defined according to the following

Proposition 9 (Unique Equilibrium Liquidity) Taking functions $q^{\prime}, \varphi^{\prime}, \psi^{\prime}, \varsigma^{s^{\prime}}, \varsigma^{i \prime}$ as given, there exists a unique functions continuous $q(s), \varphi(s), \psi(s), \varsigma^{s}(s), \varsigma_{t}^{i}(s)$ satisfying the conditions in Propositions 3,7 and 8 .

Proof. See appendix K.

Proposition 9 states that the equilibrium conditions of the model define a self-map in $q^{\prime}, \varphi^{\prime}, \psi^{\prime}, \varsigma^{s^{\prime}}, \varsigma^{i \prime}$. In the simulations carried out in the following section we find that the comparative statics are highly non linear. A recursive competitive equilibrium, is a fixed point of the operator implicitly defined in Proposition 9. Finding a contraction property for the whole system may be hard so we proceed by parts. The system has a monotone property that allows us to compute equilibria.

Proposition 10 (Unique Equilibrium Liquidity) The mapping defined by equilibrium of this economy satisfies Schauder's fixed point theorem. A fixed point $q^{*}, \varphi^{*}, \psi^{*}, \varsigma^{s . *}, \varsigma^{i . *}$ may be computed by repeated iteration.

Proof. See appendix L.

An additional property of this equilibrium is that,

Corollary $2 q^{*}, \varphi^{*}, \psi^{*}, \varsigma^{s . *}, \varsigma^{i . *}$ and I are continuous functions of the aggregate state $s$.

Proof. See appendix L.

The proof follows immediately from continuity of $q$ in Proposition 2 in the appendix, and the characterization of $\varsigma^{s}$ and $\varsigma^{i}$ in Proposition 7. With this characterization, we are ready to simulate this economy and study it. Before we proceed to this step, we take a detour to explain the intuition behind the liquidity channel. 


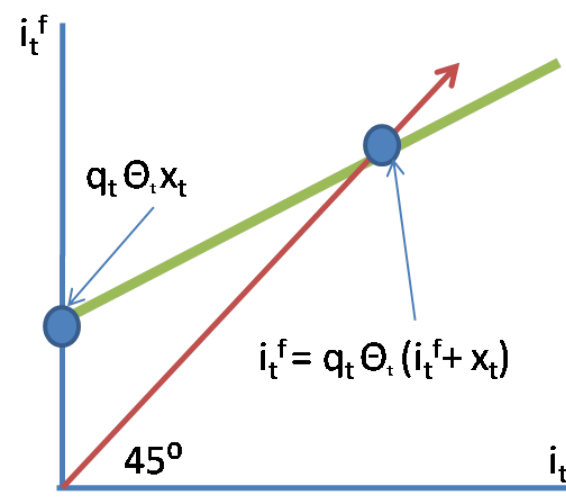

(a)

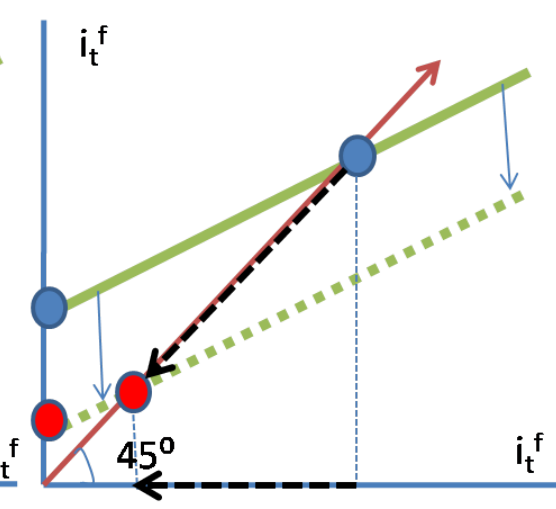

(b)



(c)

Figure 3: Liquidity constraints The left panel shows how borrowing constraints impose a cap on the amount of equity that can be sold to finance a down payment. The middle panel shows how liquidity shocks affect the amount of resources available as a down payment. The right panel shows the price effect of the shock which may or may not reinforce the liquidity shock.

\subsection{Explaining the Liquidity Channel}

A brief digression on the role of $x_{t}$ is useful before studying the equilibrium. Whenever $q_{t}>1$, external financing allows i-entrepreneurs to arbitrage. In such states, the entrepreneur wants to finance investment projects as much as possible. We have already shown that when the constraints are binding, the entrepreneur owns the fraction $(1-\theta)$ of investment while he finances only $\left(1-q_{t} \theta\right)$. If he uses less external financing he misses the opportunity to obtain more equity.

To gain intuition, let's abstract form the consumption decision and assume that the entrepreneur uses $x_{t}$ to finance the downpayment. Therefore, $i_{t}^{d}=x_{t}$. The borrowing constraints impose a restriction on the amount external equity that may be issued, $i_{t}^{s} \leq \theta i_{t}$. External financing is obtained by selling $i_{t}^{s}$ equity at a price $q_{t}$ so the external funds for the investment project are $i_{t}^{f}=q_{t} i_{t}^{s}$. Since $i_{t}=i_{t}^{f}+i_{t}^{d}$, external financing satisfies,

$$
i_{t}^{f} \leq q_{t} \theta\left(i_{t}^{f}+x_{t}\right)
$$

Figures 3 describes the simple intuition of the liquidity channel. The figure explains how changes in the amount $x_{t}$ of sold equity affect overall investment by restricting the amount of external financing. Panel (a) in 3 shows the plots of the right and left hand sides of restriction. Outside funding, $i_{t}^{f}$, is restricted to lie in the area where the affine function is above the 45-degree line. Since $q_{t}>1$, the left panel shows that the liquidity constraint imposes a cap on the capacity to raise funds to finance the project. Panel (b) shows the effects of a decreases in $x_{t}$ without considering any price effects. The fall in the down-payment reduces the intercept of the function defined by the right hand side of Figure 3. External funding therefore falls together with the whole scale of the project. Since investment falls, the price of $q$ must rise such that the demand for saving instruments fall in accordance to the drop in supply. The increase in the price of equity implies that the amount financed externally is higher. The effect on the price increases the slope and intercept which partially counterbalances the original effect. This effect is shown on the right hand side of Figure 3. When the distribution of capital quality falls, $x$ falls jointly with an increase in $q$ and a fall in investment $i$. In equilibrium, the effect is internally consistent. 


\section{Simulations}

\subsection{Calibration}

The goal of this section is to test the model under a standard calibration by comparing its predictions with some empirical facts. The model period is a quarter. As in Campanale et al. [2009] the persistence of technology shocks is set to $\rho_{A}$ is set to 0.95 and $\sigma_{A}$ to 0.016 to match the persistence and volatility of the Solow residual. The weight of capital in production $\alpha$ is set to 0.36 is standard. The average depreciation of capital, is set to $6 \%$ in annualized terms so $E_{\phi}[\lambda]=0.991$. Together, these parameters induces average net returns to capital that range from 4 to 1 per cent a quarter depending on point of the state space. The probability of investment opportunities $\pi$ is set to 0.03 so that the expected time of arrival between investments is around 6 quarters. Finally, $\theta$ is set to $1 / 20$ which by considering the arrival of investment opportunities yields an average leverage ratio per firm of about $1 / 3$. There is less consensus about parameters governing preference. Campanale et al. [2009] assign a very low value to $\sigma$ based on experimental evidence whereas Angeletos [2007] argues that empirical work based on asset pricing biases the estimate downwards and sets the parameter to 1 . We set it to 0.9 . To calibrate $\gamma$, we follow the estimates of Vissing-Jørgensen and Attanasio [2003] which suggest a value of around between 5 and 10 for the entrepreneur fraction of the population. A higher value of 20 is assigned to the CRRA parameter of workers based on the asset pricing literature when we evaluate welfare. For an economy with production, Tallarini [2000] shows that this parameter must be much higher to match the equity premium so our model should underpredict it.

The Frisch-elasticity is determined by the inverse of $\nu$ which is set to 3 . We take this parameter as an average over several papers. Finally, $\bar{\omega}$ shifts the supply of labor multiplicatively so it acts as an implicit aggregator. The parameter is set to 5 so that the fraction of worker consumption is $50 \%$ of total consumption. These parameters determine the elasticity of returns to capital the capital stock. $\beta$ is set to 0.976 based on the complete market benchmark economy so that in a balanced growth environment (with $2 \%$ growth), steady state returns are $4.5 \%$.

Moskowitz and Vissing-Jørgensen [2002] estimate the volatility of private equity returns based on its cross-section distribution. We calibrate the distributions $f_{\phi}$ as a family of Beta distributions whose domain is stretched to be between 0.2 and 1.2. The parameters $\alpha_{\phi}$ and $\beta_{\phi}$ that characterize a Beta family are constructed to satisfy $E_{\phi}[\lambda]=0.991$. Larger values of $\phi$ are associated with less dispersion. The distributions are calibrated to replicate the maximal and minimal dispersion of equity returns Moskowitz and Vissing-Jørgensen [2002]. We impose some correlation between $A$ and $\phi$. For this purpose, $\Phi$ is partitioned into two sub-spaces. When aggregate productivity $A$, lies above it's mean, $\phi$ is drawn uniformly from above it's median value with probability $\frac{2}{3}$ and with probability $\frac{1}{3}$ its drawn from beneath its mean. These probabilities are inverted when technology shocks fall below their mean. The x-axis in figure Figure 4 is a threshold quality. The fan like curves represent the conditional expectations $E_{\phi}\left[\lambda \mid \lambda<\lambda_{*}\right]$ for each member of the $f_{\phi}$ family. The single line plot marginal valuation of keeping the threshold quality $\lambda_{*}$ for i-entrepreneurs. Liquidity is determined by the points where the valuation crosses the conditional expectation. Thus, for each $\phi$, the threshold quality is different and increases with the dispersion of $f_{\phi}$. Figure 5 compares the amount of liquidity, (the threshold in figure 4) for different values of $\mathrm{q}$ and $\phi$. Liquidity increases with a lower dispersion and with a higher value of $q$. These plots correspond to the calibrated example.

A summary of the baseline calibration and different perturbations is reported in Table 1. All of the simulations carried out for the following tests are computed using a grid of 6 elements for both 
Table 1: Calibrations

\begin{tabular}{|l|lllll|llll|lll|l|}
\hline & \multicolumn{4}{|c|}{ Preferences } & \multicolumn{4}{c|}{ Technology } & \multicolumn{4}{c|}{ Aggregate Shocks } \\
\hline & $\beta$ & $\gamma$ & $\sigma$ & $\nu$ & $\omega$ & $\alpha$ & $E[\lambda]$ & $\pi$ & $\theta$ & $\rho_{A}$ & $\mu_{A}$ & $\sigma_{A}$ & $\operatorname{corr}_{\phi, A}$ \\
\hline Baseline & 0.9764 & 10 & 0.8 & 2 & 1.1 & 0.36 & 0.995 & 0.3 & 0.04 & 0.95 & 0.0 & 0.016 & 0.66 \\
\hline
\end{tabular}

$\mathbb{A}$ and $\Phi$ and using a grid of 120 value for capital. ${ }^{15}$ The next sections describe the main business cycle and asset pricing implications.

\subsection{Results}

Figure 6 shows the behavior of $q$ and $q^{R}$ as functions of aggregate capital the endogenous state. ${ }^{16}$ Within the figure, each of the four panels corresponds to a combination of aggregate productivity shocks and a shock to the dispersion of capital quality, the exogenous states of the model. Each panel in the figure allows us to understand the interaction between the capital stock and $q$. In all panels, the first thing to observe is that for a given quality dispersion and aggregate productivity, a lower capital stock leads to a higher $q$. This follows from a higher equity demand in states where the capital stock since returns are higher be decreasing returns to scale.

The four panels show a threshold capital $K$ for which $q$ is above one. The corresponding investment functions depicted in Figure 7 show that these thresholds correspond to states with positive investment as characterized. To provide liquidity at all, the wedge between $q$ and $q^{R}$ must be positive, thus $q>1$. Each panel in Figure 7 depicts three functions corresponding to aggregate investment (solid), and investment from i and s entrepreneurs (dotted and dashed respectively). Note that the share of investment by i-entrepreneurs is much larger when investment is positive because they must satisfy the aggregate incentive compatibility constraint (18). Inside the model, i-entrepreneurs are trading old equity to finance collateral so the increase in total equity holdings is not so uneven. Figures 8 the corresponding liquidity in all cases. The lower the capital stock becomes, the higher will average returns. Not surprisingly, states with a low capital stock are characterized by higher $q$ and investment. But also, because $q$ is higher, financial terms offer better terms that lead to a larger volume of equity sold.

By comparing the top panels with the panels in the bottom we can understand the effects of aggregate productivity shocks. Aggregate productivity increases $q$ as the demand for equity is greater because productivity is autocorrelated. Naturally, investment is larger in states where productivity is higher. The corresponding liquidity is larger also in states with higher productivity. As $q$ is larger, financial firms offer better conditions but at the same time, more liquidity relaxes the constraints to carry out larger investment projects.

By comparing the left panels with right columns we get a clear idea of the effects of more dispersion in the quality of capital. Observe that when the dispersion falls, $q$ becomes smaller. In equilibrium, financial firms offer better terms for the same volume of equity sold. Observe that in spite of a higher value of $q$, and therefore, there are larger arbitrage opportunities of entrepreneurs, there is less liquidity 8. The reason is that financial firms are offering worse terms when dealing with the pools. More dispersion means that although the valuation of keeping an asset is falling for

\footnotetext{
${ }^{15}$ Matlab@ and Fortran(C) codes are provided in the author's webpage. The whole system is computed in about 40 minutes depending on the parameters $\gamma$ and $\frac{1}{\sigma}$.

${ }^{16}$ The axis can be translated to the return to capital.
} 


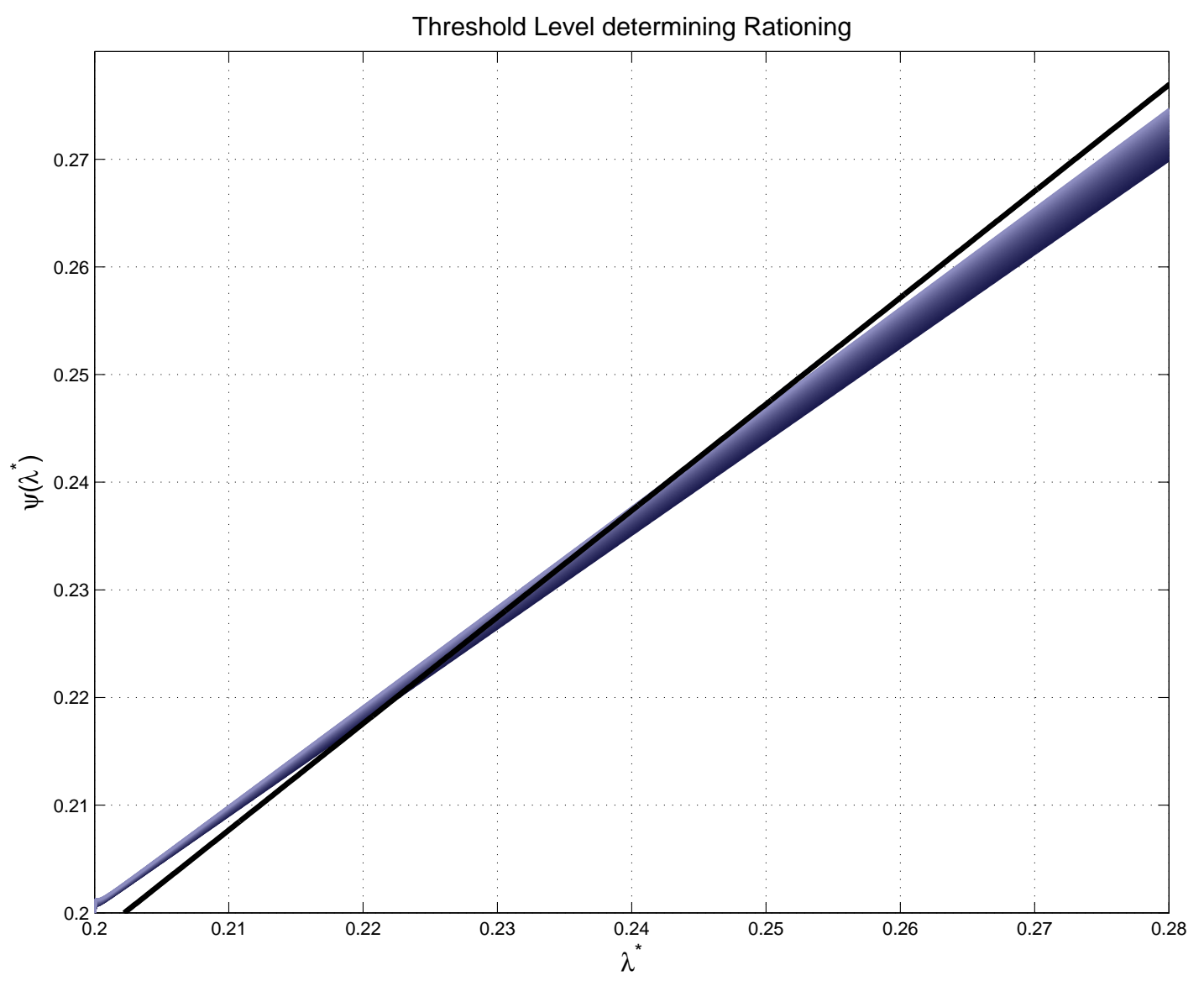

Figure 4: Threshold Qualities For an arbitrary value of q, the figure plots two function. The xaxis corresponds to the threshold liquidity. The thick line corresponds to the amount $x_{c}$ that would be required to make a particular quality the threshold quality. This function corresponds to equation 9. The thin lines plot the financial firms valuation of equity conditional on the threshold quality. These curves correspond to different $\phi$ in equation 10. A competitive equilibrium is determined by the point in which the curves meet. Assumption 2 guarantees uniqueness of thresholds and that these are decreasing in the dispersion of capital quality. 

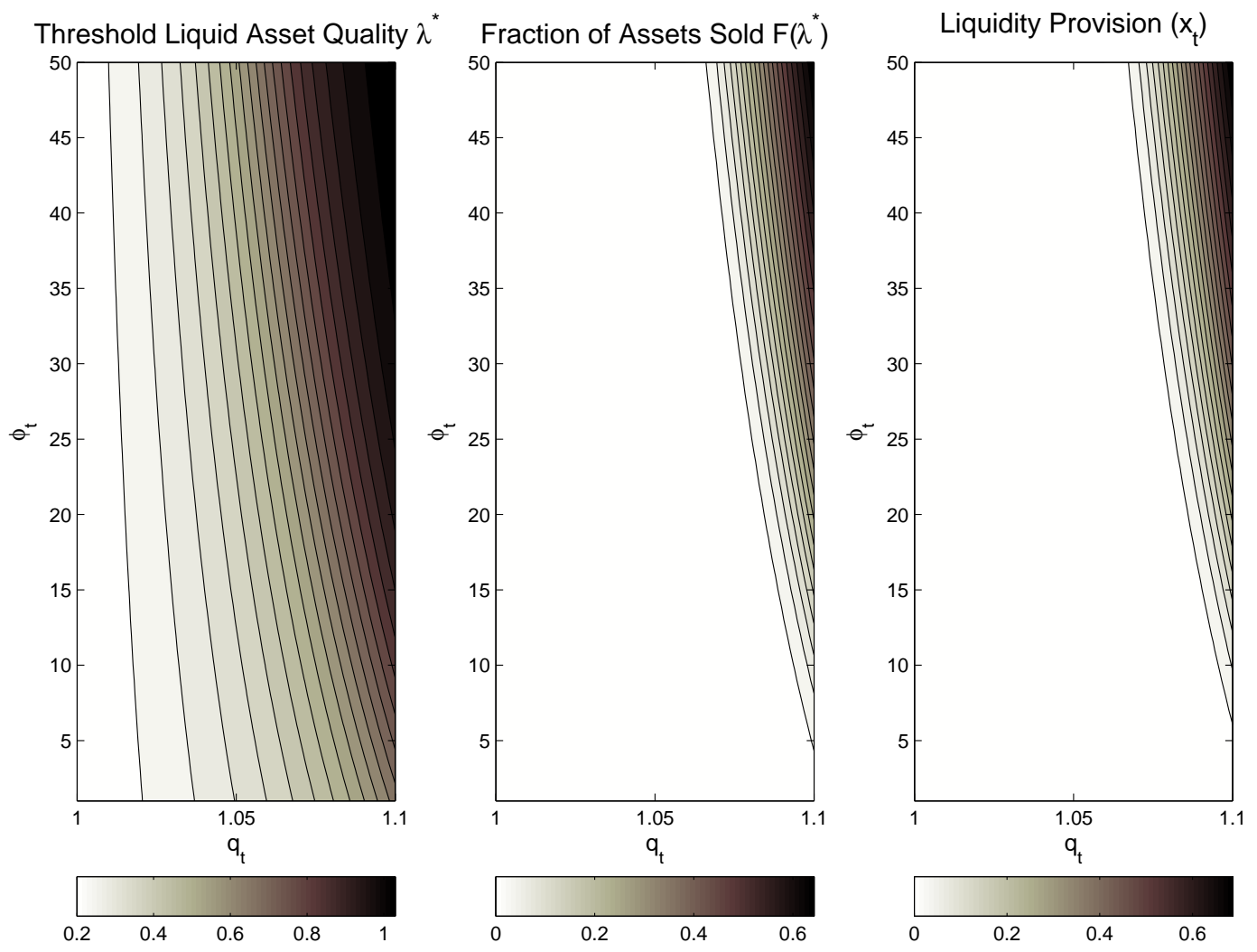

Figure 5: Sample Equilibrium Contracts From left to right, figure plots the volume of traded assets, the total value of liquid assets and the relative value of liquid assets to all assets of a typical entrepreneur. The figure shows how these measures depend on the values of $\phi_{t}$ (which measures the dispersion of capital quality) and $q_{t}$ as given by (10). 
Table 2: Results - Unconditional Moments

\begin{tabular}{|c|l|llllc|}
\hline Calibration & FracCons & \multicolumn{1}{c}{$\mathrm{q}$} & $\frac{i}{y}$ & $\varsigma_{i}$ & $\varsigma_{s}$ & $\operatorname{corr}(q, i)$ \\
\hline Baseline & 0.5966 & 1.028 & -0.0131 & 0.9736 & 0.9737 & 0.8066 \\
& & $(0.03158)$ & $(0.07464)$ & $(5.18 \mathrm{e}-005)$ & $(4.017 \mathrm{e}-005)$ & \\
\hline
\end{tabular}

Table 3: Results - Conditional Moments

\begin{tabular}{|c|l|llllc|}
\hline Calibration & FracCons & \multicolumn{1}{|c}{$\mathrm{q}$} & $\frac{i}{y}$ & $\varsigma_{i}$ & $\varsigma_{s}$ & $\operatorname{corr}(q, i)$ \\
\hline Baseline & & 1.047 & 0.03534 & 0.9737 & 0.9737 & 0.7739 \\
& & $(0.02788)$ & $(0.03625)$ & $(4.18 \mathrm{e}-005)$ & $(4.18 \mathrm{e}-005)$ & \\
\hline
\end{tabular}

sellers, the expected quality of equity for financial firms falls. It is important to mention though that this direction crucially depends on our choice of $f_{\phi}$ and results in general cannot be established, and the relation with more dispersion may even be non-monotone. ${ }^{17}$ Figures 9 and 10 show the relative change in investment and liquidity when the dispersion falls.

Tables 2 and 3 report the standard RBC-statistics from the stationary distribution of the model. The tables correspond to unconditional moments and moments conditional on liquidity constraints binding. The marginal unconditional density of the capital state and the conditional density (conditional on liquidity constraints) are painted in figure 15 in the appendix. The first column reports the occupation time during liquidity constrained states. These correspond to states with positive investment and accounts for almost $60 \%$ of the time. As expected, Tobin's q is approximately $2 \%$ larger in states where constraints bind. The final column shows how the relation between $\mathrm{q}$ and investment is smaller in states where constraints are binding. This result is similar to Lorenzoni and Walentin [2009].

The next section describes how the model the comparative statics are decomposed into RBC, limited enforcement and asymmetric information effects.

\footnotetext{
${ }^{17}$ For example, this is the case when we use a log-normal distribution.
} 


\section{Tobin Q}
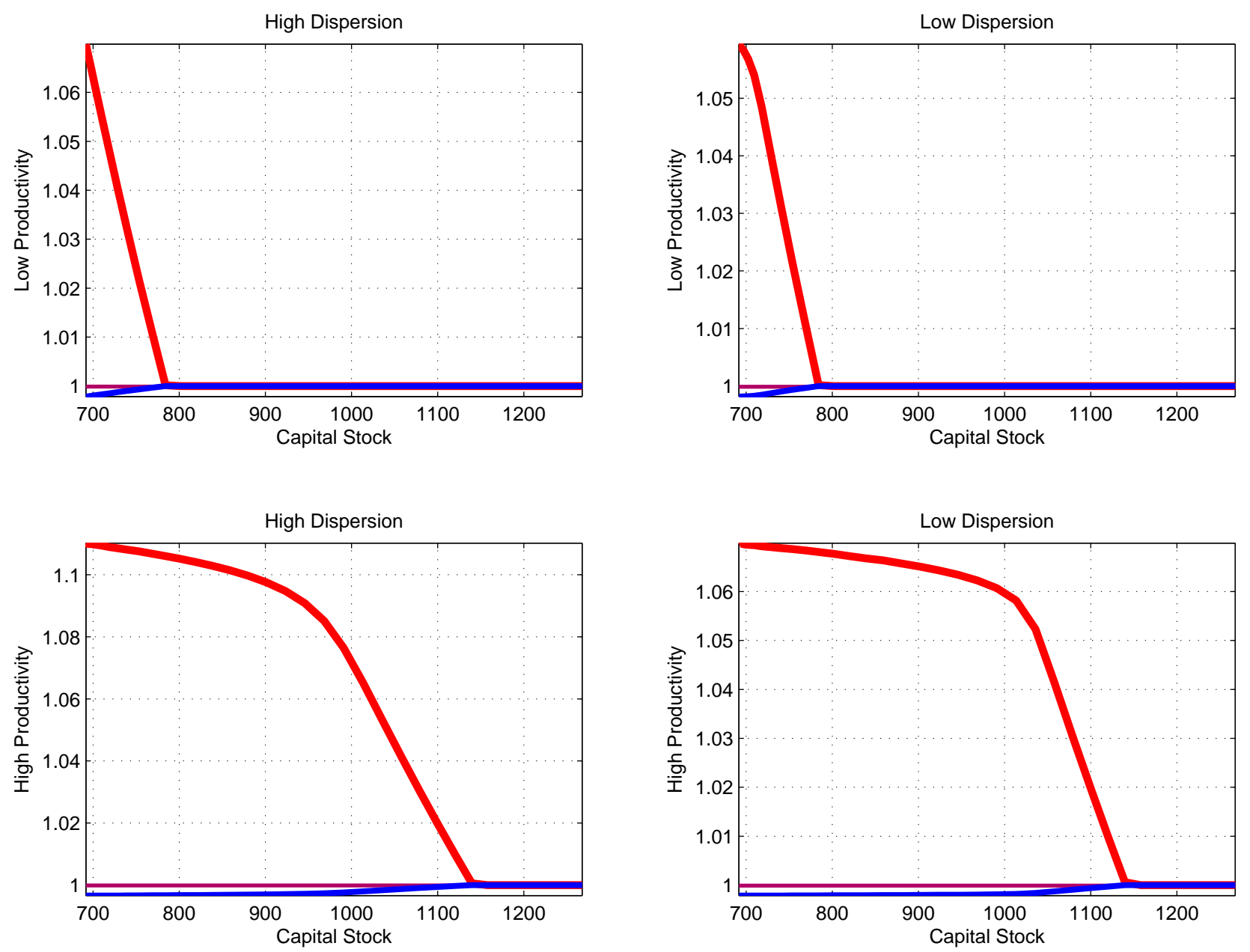

Figure 6: Value of Tobin's Q along the State Space. The figure shows the value of q (red) and $q^{R}$ (blue) as a functions of aggregate capita states for 4 combinations of aggregate productivity and liquidity shocks. The figures show that at wedge exists the higher the aggregate productivity shock or the tighter the liquidity shock. For all four cases, the wedge is greater the lower the capital stock, reflecting that liquidity matters more, when returns are higher. 
Investment Decomposion
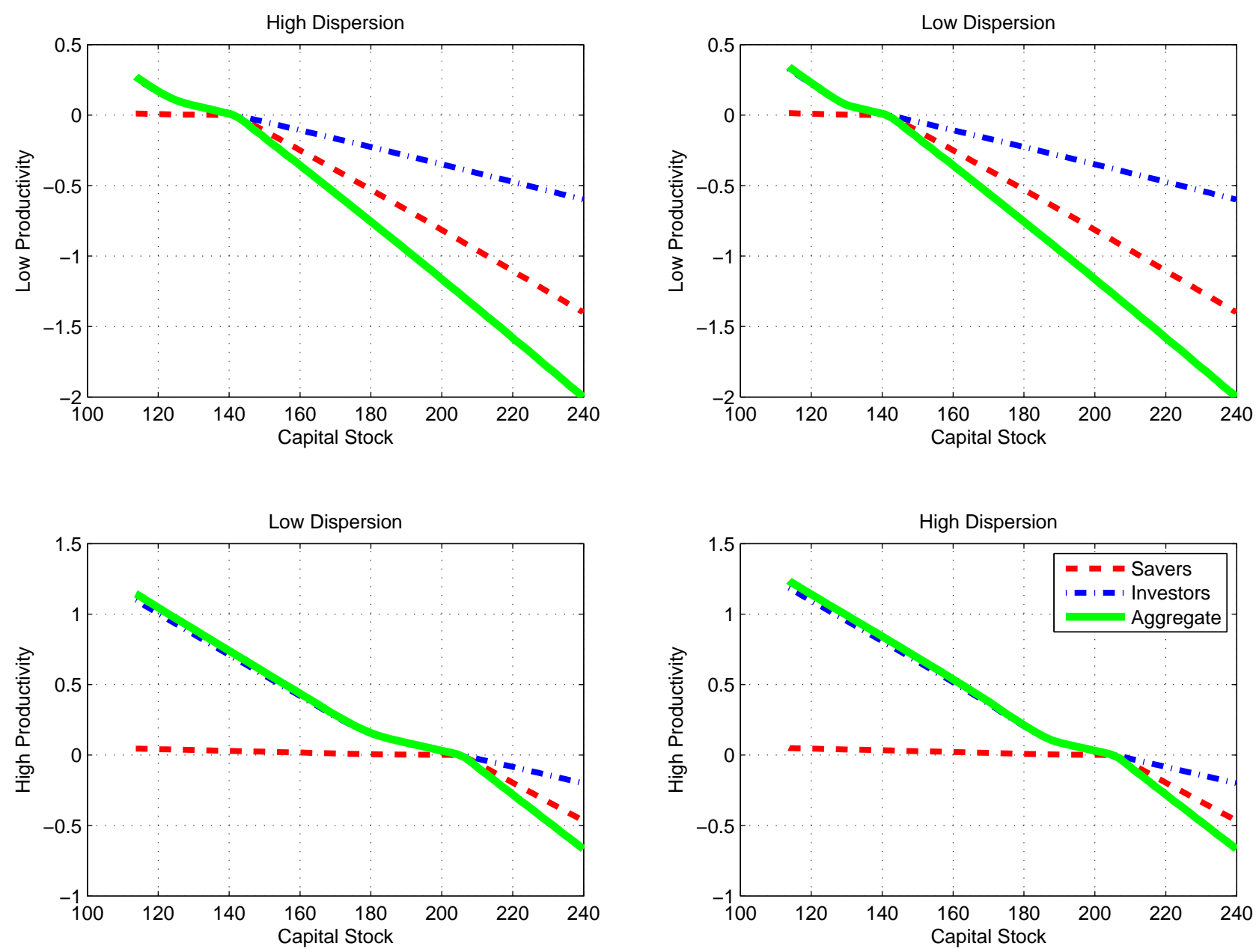

Figure 7: Investment along the State Space. The figure shows the value of the functions $i_{t}$ and $i_{t}^{c}$ as a functions of aggregate capital states for 4 combinations of $\mathrm{A}$ and $\phi$. The figures show that liquidity constraints bind the higher the aggregate productivity shock or the more disperse the capital quality. In all cases, constraints as the the capital stock is lower (returns are higher). 


\section{Fraction of Liquid Assets}
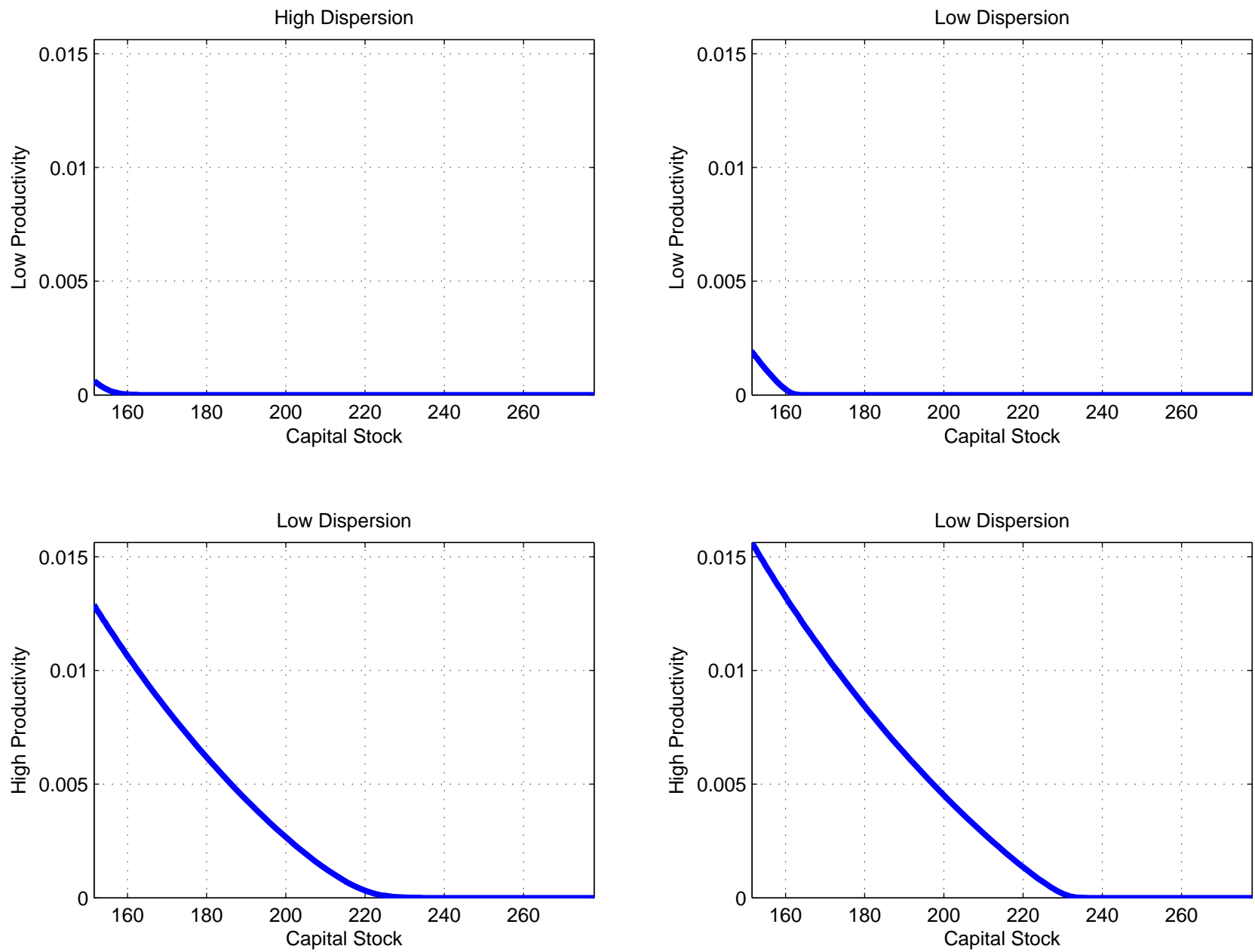

Figure 8: Fraction of Liquid Assets along the State Space. The figure shows the value of the function $\varphi$ which measure the fraction of liquid assets as a functions of aggregate capital for four states for 4 combinations of $\mathrm{A}$ and $\phi$. The figures show how the volume of assets falls as returns to capital fall. 


\section{\% Differences in Investment as Proportion of Investment with Low Dispersion High Dispersion minus Low Dispersion}
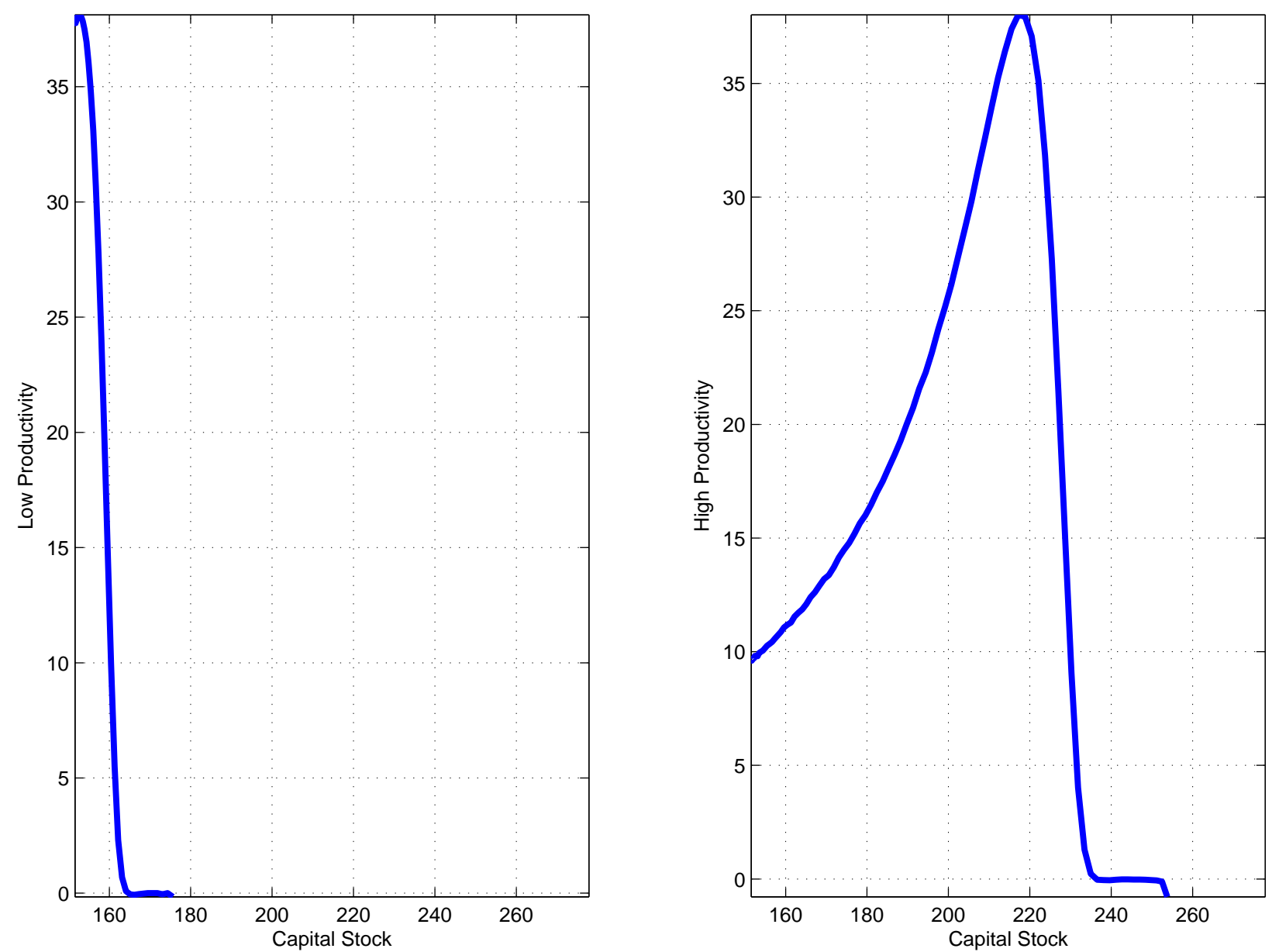

Figure 9: Investment Differences between High and Low dispersion States. The figures compare the $\%$ change in investment after an increase in the dispersion of capital. The effect only exists in states with positive investment. The left and right panels correspond to high and low productivity states. The larger scale in the left panel shows a positive interaction between aggregate productivity shocks and shocks to the dispersion of capital. 


\section{Differences in Liquidity as \% of Total Assets \\ High Dispersion minus Low Dispersion}
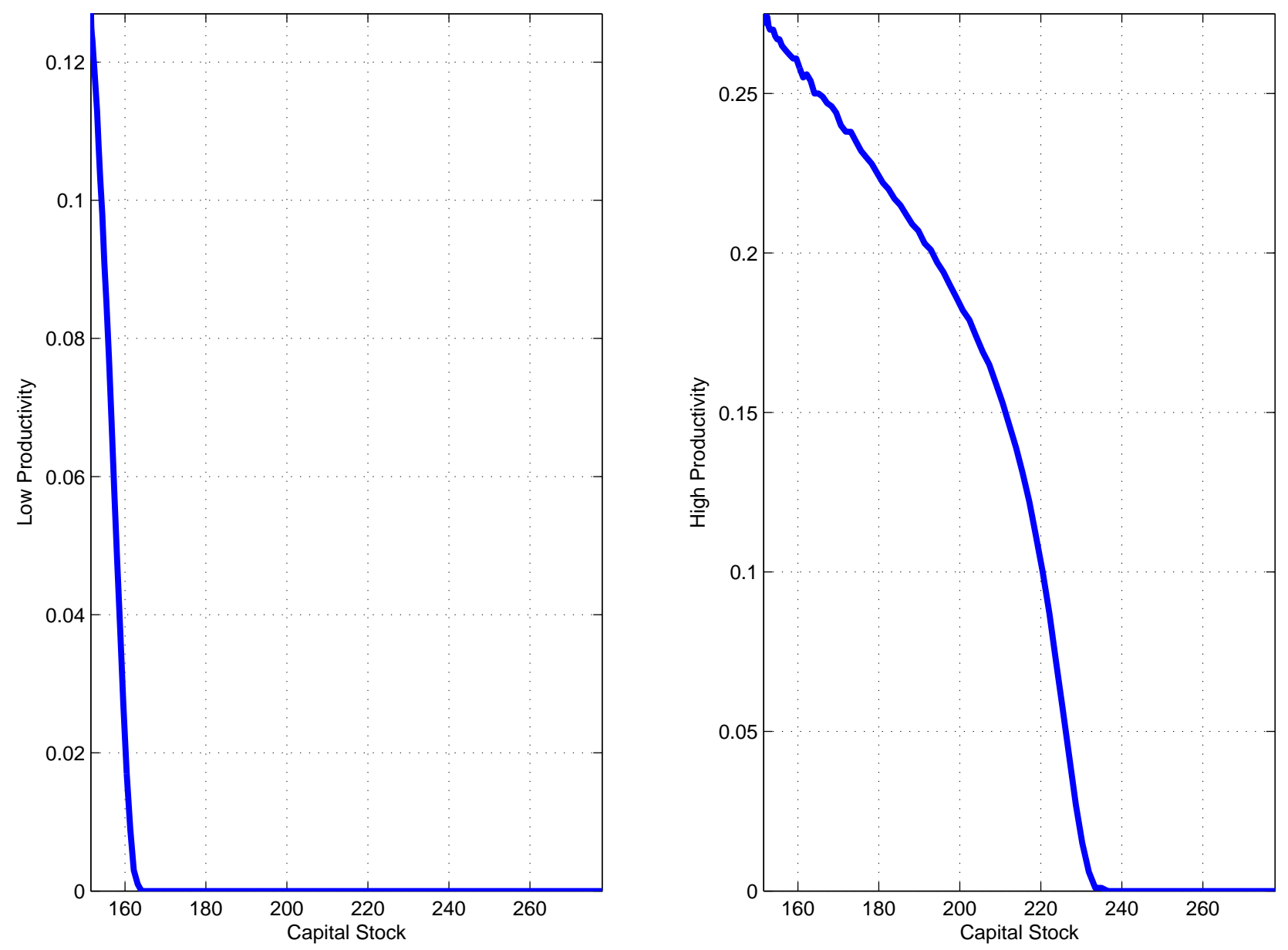

Figure 10: Liquidity Differences between High and Low dispersion States. The figure plots the change in the function $\varphi$ after an decrease in the dispersion of capital. This function measures the fraction of assets that are traded to finance the collateral constraint. 


\subsection{Decomposing Business Cycles}

This section describe how the effects of technology shocks and shocks to the dispersion of capital quality can be decomposed into standard RBC and liquidity effects. The main point of interest are the effects on aggregate investment. Recall that investment is given by condition (19). When investment is positive, we showed that the collateral constraint (6) must bind. Therefore, the effect on aggregate investment is characterized by the effect on the equilibrium demand for investment of i-entrepreneurs (16) per unit of capital:

$$
\frac{\partial I(s)}{\partial A}=\frac{\pi}{(1-\theta) K} \frac{\partial I^{i}(q)}{\partial A}
$$

The effects on investment, (and future output) of productivity shocks can be decoupled into two terms. The first term measures the standard RBC effect of productivity shocks. The second term is due to the innefficiency caused by limited enforcement. By replacing the agents policy functions and rearranging terms, we obtain the following expression for the effects of a productivity shock:

$$
\begin{aligned}
\frac{\partial I^{i}(q)}{\partial A}= & \underbrace{\left[\frac{\partial \varsigma^{i}}{\partial r\left(s^{\prime}\right)} \frac{\partial r\left(s^{\prime}\right)}{\partial A}\right]\left(r(s)+q^{i}(s) E[\lambda]\right)}_{\text {RBC effect }}+\underbrace{\varsigma^{i} \frac{\partial r\left(s^{\prime}\right)}{\partial A}}_{\text {Substitution effect }} \ldots \\
& \underbrace{+\frac{\partial q}{\partial A}[\underbrace{\frac{\partial \varsigma^{i}}{\partial q}\left(r(s)+q^{i}(s) E[\lambda]\right)}_{\text {Substitution effect }}+\underbrace{\varsigma^{i} q^{R} \varphi}_{\text {Wealth effect }}+\underbrace{\frac{\partial \varphi}{\partial q}\left(\frac{q(s) \varsigma^{i}+q^{R}(s)\left(1-\varsigma^{i}\right)}{q^{R}(s)}\right)}_{\text {Asymmetric Information effect }}]}_{\text {Wealth effect }}
\end{aligned}
$$

The first term of (21) is a direct effect that corresponds to the RBC effect. As in RBC models, technology shocks have two sub-effects. The first term depends on the elasticity of the savings function productivity $\frac{\partial \varsigma^{i}}{\partial A}$. The substitution effect reflects current and future changes productivity. Under log preferences, there is no substitution effect, so the savings function remains constant and equal to $\beta$. Here, the direction of the effect depends on the IES parameter, $\sigma$, which determines the degree of substitution between consumption and savings. The second RBC effect is a direct wealth effect. Since the economy experiences an increase in current returns, $\frac{\partial r\left(s^{\prime}\right)}{\partial A}>0$, investment increases partially by $\varsigma^{i}$ times this term.

The limited enforcement effect is measured through its effect on the price of equity, $\frac{\partial q}{\partial A}$. Without limited enforcement no such effect would exist. Proposition 9 shows that increments in productivity will cause have a positive effect on the demand for equity $\frac{\partial q}{\partial A} \geq 0$. Therefore, the limited enforcement effect has three direct sub-effects on investment by i-entrepreneurs. By affecting the relative price of equity i-entrepreneurs are relatively wealthier. Thus, limited enforcement carries a substitution effect on the savings function, $\frac{\partial \varsigma^{i}}{\partial q}$, whose strength again depends on $\sigma$. In addition, the effect on $q$, yields another direct wealth effect which equals $q^{R} \varphi$. Finally, q affects the level of liquid assets, $\frac{\partial \varphi}{\partial q}$, which we call an asymmetric information effect. The degree of liquidity increases with $\frac{\partial \varphi}{\partial q}>0$. Thus, liquidity is procyclical. Without limited enforcement, asymmetric information plays no role in the model so both effects act jointly. ${ }^{18}$. We provide a numerical example where these effects

\footnotetext{
${ }^{18}$ Kurlat [2009] establishes a relation between $q$ and distortionary taxes. He decouples the effects of productivity shocks by freezing the terms where $\frac{\partial q}{\partial A}$ appears. He then studies the overall effect limited enforcement and asymmetric information effect jointly
} 
are decoupled to quantify their importance. Overall, the message of this sections is that limited enforcement and asymmetric information amplify the business cycle effects of productivity shocks. ${ }^{19}$

A similar decomposition may be carried out to explain the effects of an increase in the mean preserving spread in the quality of capital. Again by construction we have that $\partial \sigma_{\phi} / \partial \phi>0$.

$$
\frac{\partial I^{i}(q)}{\partial \phi}=\underbrace{\frac{\partial q}{\partial \phi}[\underbrace{\frac{\partial \varsigma^{i}}{\partial q}\left(r(s)+q^{i}(s) E[\lambda]\right)}_{\text {Substitution effect }}+\underbrace{\varsigma^{i} q^{R} \varphi}_{\text {Wealth effect }}]}_{\text {Limited Enforcement effect }}+\underbrace{\frac{\partial \varphi}{\partial \phi}\left(\frac{q(s) \varsigma^{i}+q^{R}(s)\left(1-\varsigma^{i}\right)}{q^{R}(s)}\right)}_{\text {Asymmetric Information effect }}
$$

In an RBC economy, an increase in the mean preserving spread of the quality has no effects on output so this shocks have no RBC effect. On the other hand, by affecting the level of liquidity, shocks to the dispersion of capital quality carry out real effects. An increase in the mean preserving spread will reduce liquidity $\frac{\partial \varphi}{\partial \phi}<0$ due to asymmetric information. Due to limited enforcement, $q$ will increase after the shock $\frac{\partial q}{\partial \phi}>0$ because the supply of saving instruments is falling. The increase in $q$, makes i-entrepreneurs wealthier and so investment is also impacted by wealth and substitution effects. Once again, with log-utility, such effect is 0. Overall, asymmetric information and the limited enforcement effects may act in different directions. However, since overall liquidity falls, the asymmetric information effect must dominate. If the shock studied here carries information on future shocks, additional portfolio effects captured by $\frac{\partial \varsigma^{i}}{\partial \phi}$ would show-up.

By using the decomposition formula 22, Figure 11 describes the decomposition of the liquidity effect. About $1 / 3$ of the fall in investment is explained by asymmetric information. Interestingly, through limited enforcement, the increase in $q$ reduces the i-entrepreneurs' to consume. The asymmetric information effect is roughly constant with aggregate productivity which makes sense considering that aggregate productivity plays no role in determining what assets are traded in the equity pool. On the other hand, the effect of limited enforcement is lower is states with higher productivity because there is less substitution between investment and consumption.

${ }^{19}$ The formula above allows us to decompose impulse response functions into each of the five components. 
Decomposition of Fall in Investment after and increase in dispersion Limited Enforcement vs. Assymetric Information
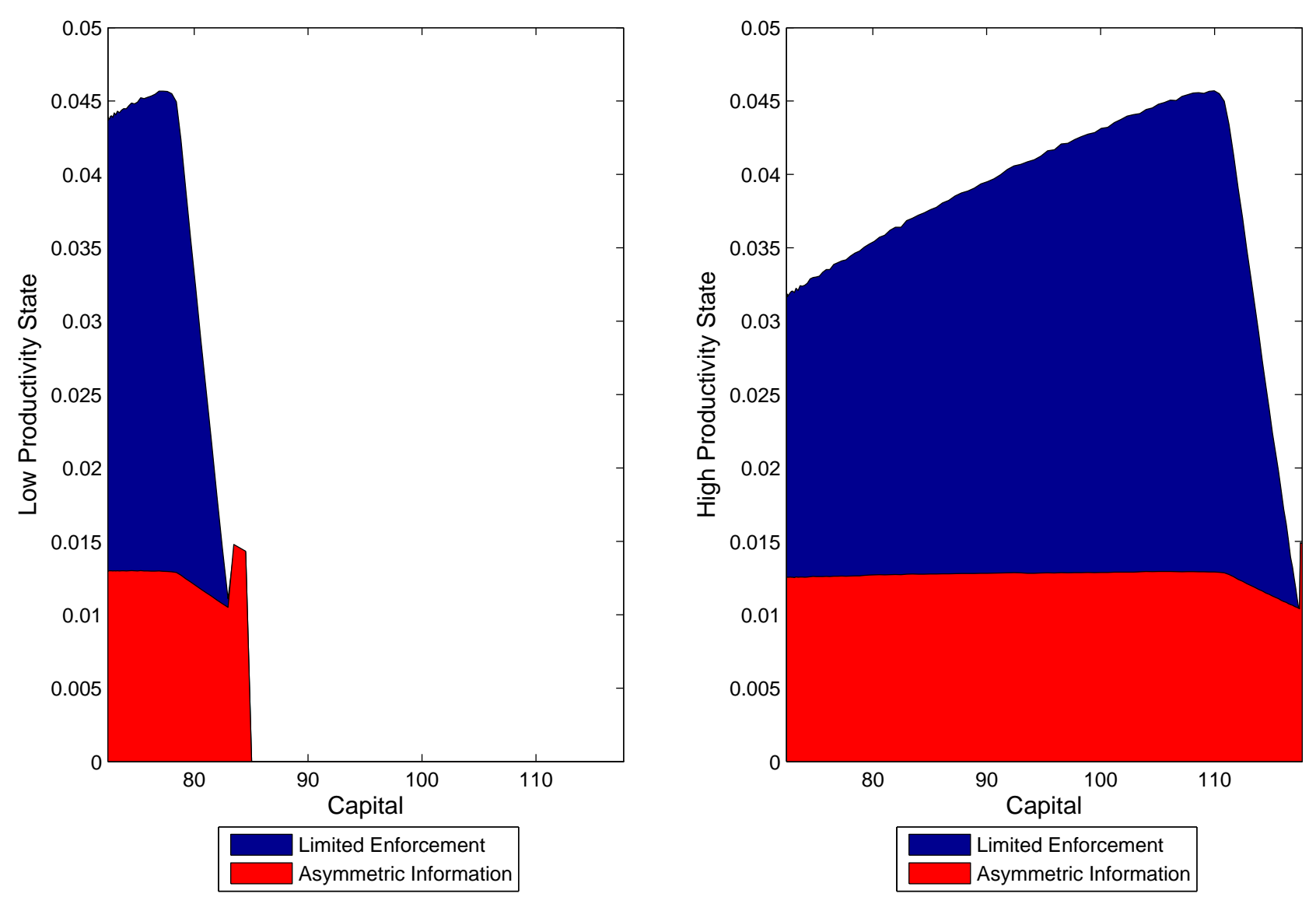

Figure 11: Decomposition of Fall in Investment. The figure compares the effects of the limited enforcement constraint and the adverse selection component. The figure shows how asymmetric information amplifies the fall in output by reducing the amount of assets sold. Asymmetric information has an amplification effect of about $1 / 3$. 


\subsection{Decomposing Asset Prices}

The advantage of studying the model under more genereal preference than log-preferences is that we can contrast the asset pricing properties of our model with other models of asset pricing with production. It is known from the asset pricing literature that in order to obtain reasonable asset prices in a model with production, there needs to be a source of variation in $q$. Following Jermann [1998], many papers use adjustment costs to provide some variation in $q$. Nevertheless, Tallarini [2000] shows that in order to replicate asset prices, asset pricing models need to incorporate preferences that distinguish the intertemporal elasticity of substitution from risk aversion. Our model provides an endogenous source of variation in $q$ and Epstein-Zin preferences. Thus, it should be in good standing to explain some asset pricing features of the data. On the other hand, its asset pricing properties provide a natural test for the variation in liquidity that the model delivers. On the other hand, the model delivers a state-dependent liquidity premium because liquidity depends on the business cycle.

As a benchmark, consider the price of riskless bond in zero net supply. Note that s-entrepreneurs would determine the price of this asset because both entrepreneur types cannot satisfy their euler equations simultaneously. Since the return to i-entrepreneurs is higher, s-entrepreneurs would be the only agents with a demand for this bond. The first order condition of the saving entrepreneur yields a pricing formula for any asset with return $R^{b}$.

$$
1=\beta E\left[\frac{\left[\mathcal{C E}\left(\left(1-\varsigma^{j}\left(s^{\prime}\right)\right)^{\frac{1}{1-\sigma}} R^{j, s}\left(s^{\prime}\right)\right)\right]^{\gamma-\frac{1}{\sigma}}}{\left(1-\varsigma^{s}(s)\right)^{-\frac{1}{\sigma}}}\left(1-\varsigma^{j}(s)\right)^{\frac{(1-\gamma)}{1-\sigma}}\left(R^{j, s}\left(s^{\prime}\right)\right)^{-\gamma} R^{b}\right]
$$

where $j$ is the entrepreneurs type next period. The stochastic discount factor here is expressed as a function of marginal propensities to consume and the return to the average portfolio:

$$
\mu\left(s^{\prime}\right)=\beta \frac{\left[\mathcal{C E}\left(\left(1-\varsigma^{j}\left(s^{\prime}\right)\right)^{\frac{1}{1-\sigma}} R^{j, s}\left(s^{\prime}\right)\right)\right]^{\gamma-\frac{1}{\sigma}}}{\left(1-\varsigma^{s}\left(s^{\prime}\right)\right)^{-\frac{1}{\sigma}}} E\left[\left(1-\varsigma^{j}\left(s^{\prime}\right)\right)^{\frac{(1-\gamma)}{1-\sigma}}\left(R^{j, s}\left(s^{\prime}\right)\right)^{-\gamma}\right]
$$

where

$$
\begin{aligned}
& E\left[\left(1-\varsigma^{j}\left(s^{\prime}\right)\right)^{\frac{(1-\gamma)}{1-\sigma}}\left(R^{j, s}\left(s^{\prime}\right)\right)^{-\gamma}\right] \\
= & {\left[\pi\left(1-\varsigma^{i}\left(s^{\prime}\right)\right)^{\frac{(1-\gamma)}{1-\sigma}}\left(R^{i, s}\left(s^{\prime}\right)\right)^{-\gamma}+(1-\pi)\left(1-\varsigma^{s}\left(s^{\prime}\right)\right)^{\frac{(1-\gamma)}{1-\sigma}}\left(R^{s}\left(s^{\prime}\right)\right)^{-\gamma}\right] }
\end{aligned}
$$

The return to a riskless bond in zero net supply is $R^{b}(s)=E\left[\mu\left(s^{\prime}\right) \mid s\right]^{-1}$ and excess returns follow the textbook formula:

$$
E\left[R^{s}\left(s^{\prime}, s\right)\right]-R^{b}(s)=-\frac{\operatorname{cov}\left(\mu\left(s^{\prime}\right), R^{s, n}\left(s^{\prime}\right)\right)}{E\left(\mu\left(s^{\prime}\right)\right)}
$$

Furthermore, the equity premium can be decomposed into two effects:

$$
=\underbrace{-\frac{\operatorname{cov}\left(\mu\left(s^{\prime}\right), r\left(s^{\prime}\right) / q(s)\right)}{E\left(\mu\left(s^{\prime}\right)\right)}}_{\text {RBC Risk Premium }}-\underbrace{\pi \frac{\operatorname{cov}\left(\mu\left(s^{\prime}\right), q^{i}\left(s^{\prime}\right) / q(s)\right)}{E\left(\mu\left(s^{\prime}\right)\right)} E[\lambda]}_{\text {Liquidity Premium }}
$$


Table 4: Results - Asset Prices Moments

\begin{tabular}{|c|clc|}
\hline Calibration & Risk Free Rate & Equity Premium & Liquidity Premium \\
\hline Baseline & 1.3285 & 0.85855 & 0.8443 \\
& $(6.141)$ & $(0.6486)$ & $(0.6482)$ \\
\hline
\end{tabular}

These effects are first an RBC risk premium component captured by $\frac{\operatorname{cov}\left(\mu\left(s^{\prime}\right), r\left(s^{\prime}\right) / q(s)\right)}{E\left(\mu\left(s^{\prime}\right)\right)}$ which reflects the inherent random nature of equity $r_{t}$. In addition there is a liquidity, which is a product of the inefficiencies in the mode. Asymmetric information effects and limited enforcement will affect $q^{i}\left(s^{\prime}\right)$ so equity has an additional source of variation. When liquidity constraints don't bind, the second term vanishes.

From the definition of returns, and Lemma (2) and the definition of returns the following relations are established:

$$
E\left[R^{i, s}\right] \leq E\left[R^{s, s}\right] \leq E\left[R^{s}\right] \leq E\left[R^{i}\right] \leq E\left[R^{i, s}\right]
$$

where the relations become equalities when $q_{t}=1$. If one thinks of $E\left[R^{s, s}\right]$ as the return to public equity then liquidity shocks provide an explanation to observe a private equity premium which disappear when liquidity constraint don't bind. We evaluate the model using this pricing formulas in the following section.

\subsection{Asset pricing tests (Incomplete Section)}

Tables 4 and 5 describe the asset pricing properties of the model for the unconditional and conditional distributions respectively. In the first column we find expected returns of a risk free bond in 0 net supply. The baseline calibration predicts returns slightly above than $1 \%$ which is close to the data. On the other hand, the standard deviation of is much larger than in the data. The reason for this excess can only be explained by the volatility of the stochastic discount factor. The stochastic discount is much more volatile than in a standard model because the agents pricing this assets are entrepreneurs. In the model, the marginal propensity to save varies with investment opportunities. This reasoning also explains why the risk-free rate is $3 \%$ higher when constraints are binding. The reason is that in non-binding states marginal propensities to save of both $\mathrm{s}$ and i entrepreneurs are identical. The equity premia also jumps in states of binding constraints. In states of binding constraints, there is variation in the price of equity so this is a natural response.

In the data, equity premia is counter-cyclical. However, the analysis shows us something important. The equity premia should be correlated positively with productivity shocks because they make the price of equity closer to binding states. This should introduce more volatility in the price and lead as to conclude that there is a force to make equity premia pro-cyclical. However, shocks to the dispersion of capital quality counterbalances this force. As we have shown, more dispersion in capital quality leads to an increase in the price of equity but at the same time, has a negative effect on output. This force counterbalances the pro-cyclical effect through productivity. Since equity premia are counter-cyclical this should teach us something about the relative effect of the liquidity channel.

Figure 12 and 13 show that the asset pricing properties for a given capital stock and multiple combinations of aggregate shocks. 
Table 5: Results - Asset Prices Moments

\begin{tabular}{|c|clc|}
\hline Calibration & Risk Free Rate & Equity Premium & Liquidity Premium \\
\hline Baseline & 4.701 & 1.345 & 1.33 \\
& $(6.803)$ & $(0.5775)$ & $(0.5776)$ \\
\hline
\end{tabular}

Rates of Return
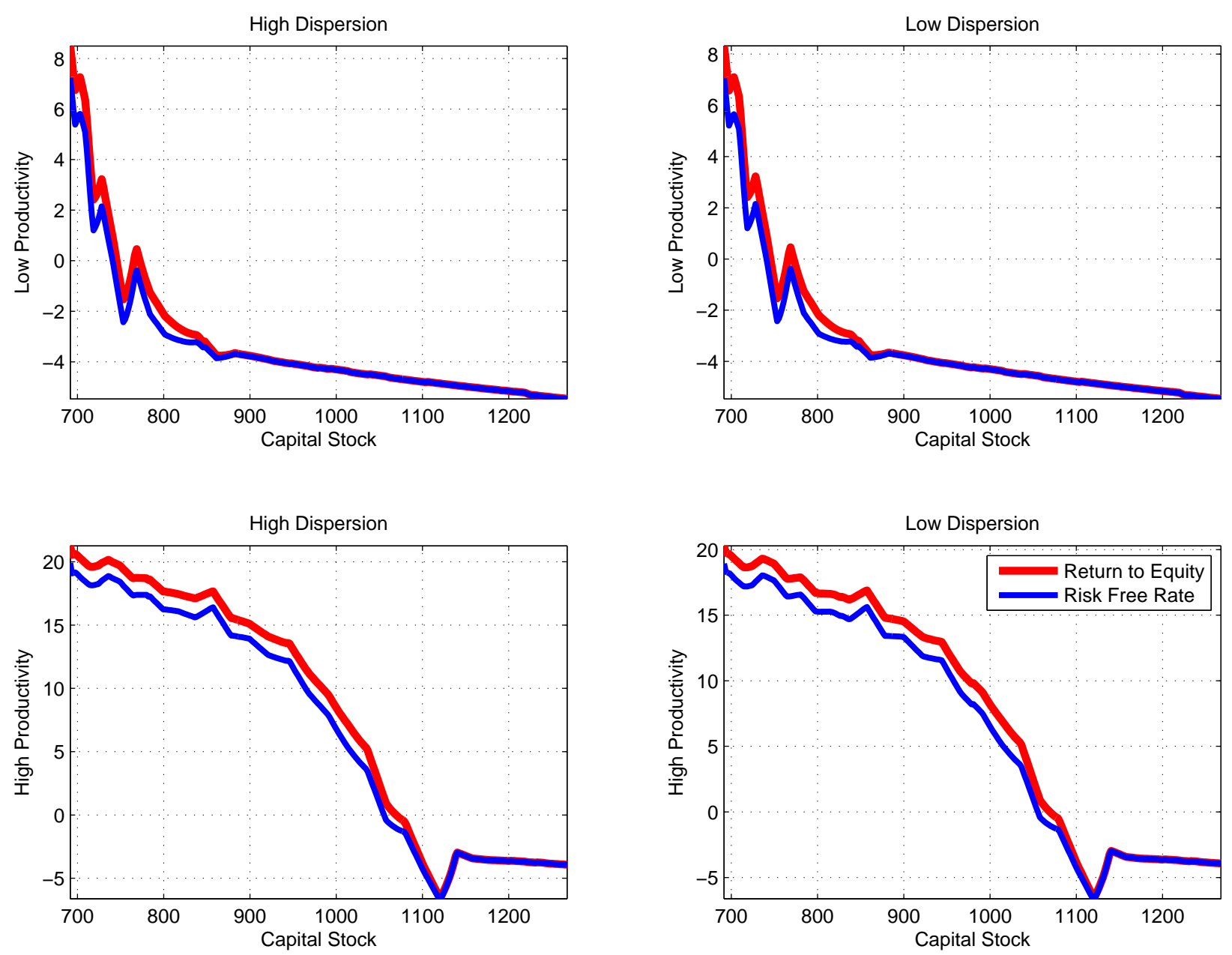

Figure 12: Return to Equity and Riskless Bond. Equity Premium and Return to Riskless Bond in the state space. 


\section{Decomposition of the Equity Premium}
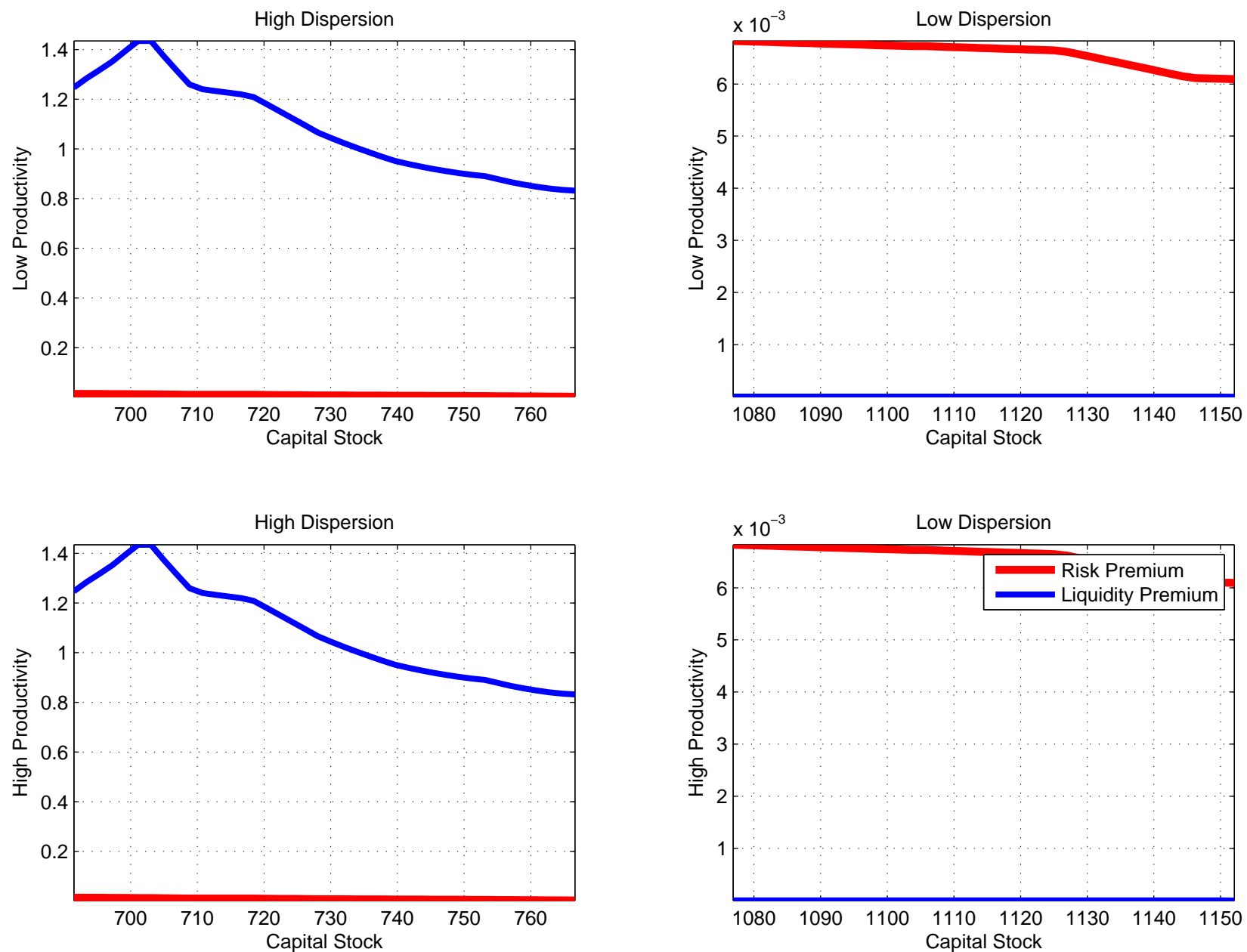

Figure 13: Decomposition of the Equity Premium. The sum of the two curves is the equity premium. The blue curve shows that the liquidity component explains almost all the equity premium. 


\section{Efficient Liquidity}

One interesting property of the model is that positive investment (gross of depreciation) requires that $q>1$. When $q=1$, no equity is sold as the incentives to participate in any pool disappear. Without liquidity, investment can no longer be efficient due to the limited enforcement constraint. Nevertheless, we can find the lowest liquidity level that makes investment efficient at all states. This function will pin down a state dependent threshold quality $\lambda_{c}$ of sold equity require to make investment efficient at all states. A contract that provides $\lambda_{c}$ liquidity would imply negative profits. The amount of goods required to make investment efficient is a measure of inefficiency. These amounts can be computed,

Proposition 11 (Liquidity Frontier) For each $(K, A, \phi) \in \mathbb{S}$, there $\exists$ ! $\lambda^{o}: \mathbb{A} \times \mathbb{K} \times \Phi \rightarrow \mathbb{R}$ such that condition (18) is satisfied with equality. Such $\lambda^{o}$ is characterized by the solution to $\lambda_{c}^{*}$ :

$$
E_{\phi}\left[\lambda(\tilde{z}) \mid \lambda(\tilde{z})<\lambda^{o}\right] F\left(\lambda^{o}\right) \equiv \frac{[(1-\pi)(1-\theta)-\theta \pi]}{\pi}\left(\varsigma^{*} r(A, K)-\left(1-\varsigma^{*}\right) E_{\phi}[\lambda]\right)
$$

and where $\varsigma^{*}$ is the fixed point of Proposition 7 evaluated at $q(s)=1$.

The amount of outside funding necessary to implement this allocation is,

$$
L^{o}=\lambda^{o}-E_{\phi}\left[\lambda(\tilde{z}) \mid \lambda(\tilde{z})<\lambda^{o}\right] F\left(\lambda^{o}\right)
$$

per unit of capital.

Proof. See appendix M.

The proposition describes the threshold capital quality $\lambda_{c}^{*}$ needed in order to provide enough liquidity in the model. The interpretation for this quantity is simple. $\left[\varsigma^{*}\left(r(A, K)+E_{\phi}[\lambda]\right)-E_{\phi}[\lambda]\right]$ is the demand for investment by all entrepreneurs (both entrepreneurs are equal when $q=1$ ) when $q=1$ (per unit of equity). The first term is the demand for equity holdings for future periods $\varsigma^{*}\left(r(A, K)+E_{\phi}[\lambda]\right)$ and $E_{\phi}[\lambda] \leq 1$ is the amount that remains undepreciated from previous periods. $(1-\pi)(1-\theta)-\theta \pi$ measures the excess quantity demanded in equilibrium. For each unit of investment that each entrepreneur wish to invest, $(1-\pi)(1-\theta)$ is the measure of s-entrepreneurs times the fraction of investment that they must own to make investment incentive compatible. $\theta \pi$ is the counterpart for s-entrepreneurs. $\pi$ is in the denominator because liquidity in the left hand side is per capita (in i-entrepreneurs). Thus, to satisfy the constraint in 18, i-entrepreneurs must transfers equity to s-entrepreneurs. This are their liquid assets. This in turn, implies an implicit contract where financial firms are subsidized.

There are several lessons obtained from this proposition. First, required liquidity is greater as the return to capital $r(A, K)$ are greater (compare this to ? where liquidity shocks are exogenous). This function, as increasing in productivity, $A$, and decreasing the capital stock, $K$, due to decreasing returns to scale. A second important lesson is that endogeneity in the source of liquidity is very important. In KM there is a threshold liquidity shock that activates the constraints. In this version, whenever investment, in this case, $\varsigma^{*} r(A, K)-\left(1-\varsigma^{*}\right) E_{\phi}[\lambda]$ is positive, there is liquidity requirement, namely, $E_{\phi}\left[\lambda(\tilde{z}) \mid \lambda(\tilde{z})<\lambda_{c}\right] F\left(\lambda_{c}\right)$. Nevertheless, if $q_{t}=1$, there is no contract that provides liquidity $\lambda_{c}=\lambda_{L}$. Thus, we have proved by contradiction that,

Proposition 12 (Liquidity Quantity) In order to have positive investment for at least s-entrepreneurs, $q>1$. Thus, positive investment is always inefficient. 
This proposition is important because it provides us with a measure of the magnitude of the liquidity requirements in this economy. First, observe that $[(1-\pi)(1-\theta)-\theta \pi] \in[-1,1]$. Moreover, since $\varsigma_{t}^{s} \cong 1$, because it is a marginal propensity to save, $r(A, K)$ should be close to $\varsigma^{s}(s)\left(r(A, K)+E_{\phi}[\lambda]\right)$. This number should be small, (and more so the lower the frequency of investment in the model). Finally, since $E_{\phi}[\lambda] \cong 1$, we have that the liquidity requirements are not very large,

$$
\left.\varphi^{*}(A, K)\right|_{q_{t=1}} \doteq \frac{r(A, K)}{\pi}
$$

as a fraction of capital, but can be several times the value of capital share of output. Thus, seen as a fraction of capital sold, the quantity is very small. For example, assuming that an average firms invests 1 every 1.5 years, and a quarterly return of $1 \%$, implies that $\varphi^{*}$ is close to $3 \%$. Thus, $F\left(\lambda_{c}\right)$ should be also close to $3 \%$, (again if $\lambda_{L}$ is close to 1 ). This suggests that in order to bind, an even smaller fraction of capital must be liquid, (considering the general equilibrium effects). Thus, capital must be very illiquid. In turn, seing this amount in turn of GDP, it can be large and in fact exceed total output. The amount required is multiplier $\frac{1}{\pi}$ times the factor share of capital $r(A, K)$. The next two sections describe the business cycle and asset pricing implications of the model.

In the model, investment is inefficient because entrepreneurs lack enough liquid assets to fully exploit investment opportunities. We showed that equity is liquid only in states where $q>1$. In turn, investment opportunities are fully exploited when investment is equal 1.Equity is illiquid when $q=1$ essentially because asymmetric information would make financial firms face losses from their activity. A natural question is therefore, what is the subsidy required by financial firms to prevent them from facing losses. This section shows how to compute the financing gap required by financial firms that would enable the economy to run efficiently as in the standard RBC economy.

An efficient economy will have $q=1$. Without subsidies, the competitive contracts we derived would have to satisfy $\lambda_{*}=\min \left\{1, x_{*}\right\}$ and $x_{*}=E_{\phi}\left[\lambda(\tilde{z}) \mid \lambda(\tilde{z}) \leq \lambda_{*}\right]$. Obviously there is no solution to this pair of conditions. To provide i-entrepreneurs with an amount $x^{o}$, equity sold by financial firms should be subsidized in the amount $\tau$ such that $\left(\lambda^{o}, \tau\right)$ to solve:

$$
\lambda^{o}=\min \left\{1, x^{o}\right\} \text { and } x^{o}=\left(1+\tau^{o}\right) E_{\phi}\left[\lambda(\tilde{z}) \mid \lambda(\tilde{z}) \leq \lambda_{*}\right]
$$

An optimal degree of liquidity will be such that $x^{o}<1$. Therefore,

$$
\left(1+\tau^{o}(s)\right)=\frac{x^{o}}{E_{\phi}\left[\lambda(\tilde{z}) \mid \lambda(\tilde{z}) \leq x^{o}\right]}
$$

Thus, we can map a desired level of liquidity to a subsidy amount via the equation above. In turn, for each point in the state space we would have to compute the value of the liquidity frontier. Using Proposition 11 we can characterize the subsidy

$$
\tau^{o}(s)=\left(\frac{x^{o}(s)}{E_{\phi}\left[\lambda(\tilde{z}) \mid \lambda(\tilde{z}) \leq x^{o}(s)\right]}-1\right)
$$

where

$$
x^{o}(s) F\left(x^{o}(s)\right)=\frac{[(1-\pi)(1-\theta)-\theta \pi]}{\pi}\left(\varsigma^{*} r(A, K)-\left(1-\varsigma^{*}\right) E_{\phi}[\lambda]\right)
$$

We can compute the state-dependent subsidy by solving first $x^{o}(s)$ and then use it to obtain $\tau^{o}(s)$. Figure 14 plots $\tau^{o}(s)$ as function of the capital state (returns) for 4 exogenous states. It is more expensive to implement first best outcomes in periods in which returns are higher because 
the liquidity needs are higher. Agents want to invest more in such states. More importantly, the degree of subsidy is much higher during periods where quality dispersion is greater.

This section is obviously for illustrative purposes. It is simply meant to reflect the sources of outside funding that financial firms would need in order to implement first best outcomes. The analysis is purely positive. Nevertheless, as with insurance markets, government intervention can be justified to improve the efficiency of the collateral market.

\section{Introducing Financial Risk Capacity}

Thus far financial firms played a trivial role in the model in the sense they there is no need to model them explicitly. This section extends the framework to study a more complex financial structure. Here, the financial sector's capacity to undertake risk will be governed by an endogenous state variable $^{20}$. This extension is important for several reasons: first, in the model, only the dispersion of quality determines the degree of liquidity. From an intuitive point of view, we should expect a relationship between the reserves of the financial sector and the degree of liquidity. When the financial system must respect a solvency constraint and faces risk in its operations, reserves act as a buffer-stock to satisfy ex-post solvency constraints. With out reserves, financial firm's must take lower risks so its financial risk capacity falls. A second for this extension is to introduce dispersion in the model. If they are to explain an important variation in the business cycle, we should expect them to have persistent effects. In the model, dispersion shocks because they are i.i.d. By introducing a financial sector, we can relate the degree of liquidity to the financial sector which also causes persistence in the shock.

Typically, the literature has focused on shocks to the supply and demand of funds provided from the private sector to financial firms. There has been minor work in relating how surprising shocks to the financial system may affect future attitudes towards risk and how it may impact attitudes towards risk in later stages.

There are some challenges in though: first, to have real effects the financial sectors profit, (which so far were 0 for all states) must face some randomness. To be surprised after they sign contracts, the market for financial contracts at the signing stage must not reveal information. For this purpose, two ingredients are introduced into the model. First, financial firms now cannot perfectly observe $\phi$ every period. Instead, $\phi$ is known once contracts have been offered. Therefore, since the zero profit condition can no longer be imposed ex-post, we introduce a second element: limited liability on the side of financial firms will impose an ex-post condition which states that financial firms must honor their contracts at every possible node. This ex-post condition will affect the level of risk capacity which magnifies the problem of asymmetric information in the equity market. This ex-post condition depends on a new state variable which is broadly interpreted as the financial sector capacity but a simplistic interpretation is the financial sectors net worth. We abstract from lending among financial firms but treat them as competitive.

Financial firms conform a unit continuum where and are identified by $\omega \in[0,1]$. They are again risk neutral, are competitive and have the objective of maximizing intertemporal dividends. In addition, they face limited liability and therefore, must satisfy a solvency constraint at the end of every period. The individual state for a financial firm $\omega$ is $B(\omega)$ which corresponds to an amount of equity it holds from entrepreneurs. The aggregate state becomes $s=A \times \phi \times K \times b$ where $b$ is the relative size of financial firms reserves and defined as,

\footnotetext{
${ }^{20}$ ? explores several government policies in the context of a simpler version of this model.
} 


\section{Liquidity Requirements}
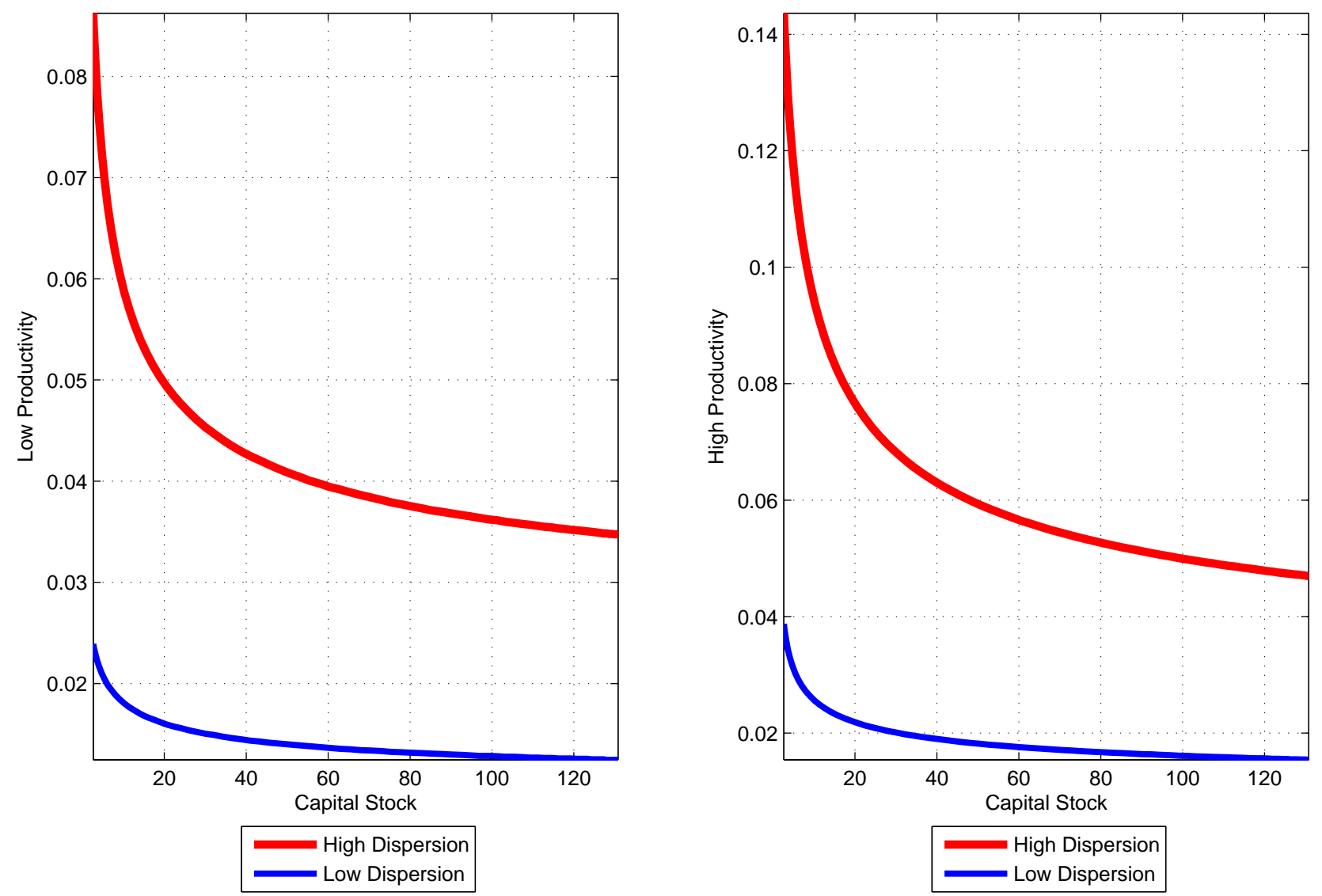

Figure 14: Liquidity Requirements. The figure plots the subsidy per trade to financial firms that is required to return to the efficient (RBC) economy. One can observe that the requirements are larger as productivity is higher. More strikingly, the degree of subsidy is substantially much higher when the asymmetric information is more severe. 


$$
b \equiv \frac{\int B(\omega) d \omega}{K}
$$

The timing is similar to the one presented before but isomorphism between the sequential and simultaneous timing protocols can no longer be established. At the first stage, financial firms observe the previous state of the economy $s$ and offer competitive contracts which we describe below. We assume but that entrepreneurs do know the distribution of qualities. Contracts and distributions determine the equilibrium amount of liquidity. During the second stage, financial profits are determined by the contracts offered and the realization of quality. At this stage, financial firms decide on a dividend policy which together with the liquidity of assets determines aggregate investment.

\subsection{Signing Stage}

At this stage, financial firms form predictions based on the previous state to offer competitive contracts. As before, they competitive contracts that specify a quantity of equity in exchange for an amount of goods. Entrepreneurs observe these contracts and sign contracts that maximize their amount of equity by the end of the period. The i-entrepreneur's policy at stage 1 will again be characterized by Proposition 9. Therefore, for a given quantity $x_{c}$ an asset of quality $\lambda(z)$ is sold during state $s$ if:

$$
\lambda(z) \leq \lambda^{*}\left(x_{c}\right) \text { where } \lambda^{*} \text { solves } x_{c}=q^{R}\left(s, x_{c}\right) \lambda^{*}\left(x_{c}\right)
$$

Notice now that the price function $q^{R}\left(s, x_{c}\right)$ is no longer a function of the current state only but also a function of $x_{c} . x_{c}$ will determine the banks profits which in turn, will affect their decision to liquidate assets. $x_{c}$ is determined by equilibrium in the financial markets. Given the equilibrium contract conditions, the scale-adjusted profits of financial firms are given by a function $u\left(x_{c}, s\right)$ :

$$
u\left(x_{c}, s\right)=q\left(x_{c}, s\right) E\left[\lambda \mid \lambda<\lambda^{*}\left(x_{c}\right)\right]-x_{c}
$$

Equilibrium Financial Contracts: Taking into account the current state $s$, financial firms offer competitive contracts by predicting the distribution of capital quality. In order to participate in the market, contracts must be ex-ante profitable so it is required that $E\left[u\left(\phi, x_{c}, s\right)\right] \geq 0$. The profits for financial firm are $u\left(x_{c}, s^{\prime}\right)$ times the scale of contracts it offered which normalize to $M\left(x_{c}, s\right) B(\omega)$, $M \geq 0$. In addition, financial firms must be able to satisfy two ex-post conditions obtained from the limited liability constraint. That is,

$$
\left[E\left[\lambda \mid s^{\prime}\right]+M\left(x_{c}, s\right) u\left(x_{c}, s^{\prime}\right)\right] \geq 0 \text { for } \forall s^{\prime}
$$

. Since, $u\left(x_{c}, s_{H}\right) \geq u\left(x_{c}, s_{L}\right)$ for $\phi_{L} \in s_{L}, \phi_{H} \in s_{H}$, this condition is equivalent to imposing:

$$
M\left(x_{c}, s_{L}\right) u\left(x_{c}, s_{L}\right) \geq-E\left[\lambda \mid \lambda<\lambda_{c}\left(x_{c}\right)\right] \text { for } s_{L} \text { such that } \phi_{L} \in s_{L}
$$

In previous sections, we derived the unique competitive contract by departing from a definition of equilibrium competitive contracts. The derivation here is similar but instead of imposing an ex-post 0 profit condition. Here, the derivation is similar except that we now impose $E\left[u\left(\phi, x_{c}, s\right)\right] \geq 0$ and condition 27. The derivation of a unique contract is analog to the one carried in the previous section so we omit a proof.

Condition 27 lies at the heart of the mechanism. The ex-post solvency constraints in particular states in which it may potentially not be met will limit the capacity of baring risk. Thus, contracts here must imply a lower degree of liquidity because they must imply a positive wedge between the 
the average value of $E\left[\lambda \mid \lambda<\lambda_{c}\left(x_{c}\right)\right]$ and the transfer to the entrepreneur. This happens because financial firms must guarantee no losses in case of a bad distribution draw. Thus, a number of reserves will affect the financial risk capacity.

Aggregate equilibrium contracts must be the ones that maximizes the price per unit of equity subject to the constraints. Thus, the value is determined by $x_{c}$ :

Proposition 13 (Equilibrium Contract 2) The equilibrium contract is determined by the price $x_{c}$ that solves

$$
E\left[u\left(x_{c}, s\right)\right]=0 \text { if } u\left(x_{c}, s_{L}\right) F\left[\lambda_{c}\right]>-E\left[\lambda \mid s_{L}\right] \text { b for } s_{L} \text { such that } \phi_{L} \in s_{L}
$$

and otherwise

$$
u\left(\phi_{L}, x_{c}, s\right) F\left(\lambda_{c}\right)=-E[\lambda] b
$$

For every state $s$ there is a unique $x_{c}$, satisfying the characterization of $x_{c}$ and the equilibrium conditions. Thus, $x_{c}(s)$ and the value of financial profits is determined by $s$, so we now omit $x_{c}$ and defined the per contract profits by $u(s)$. The equilibrium contracts signed will determine the value function at the signing stage. This value function will therefore take the form $W^{1}(B, s)=$ $E\left[W^{2}\left(B, u\left(s^{\prime}\right)\right) \mid s\right]$.

Finally, the set of offered contracts may not exactly match the amount the amount of contracts demanded. Thus perfect randomization implies the following conditions. Assuming that financial firms offer contracts accepting this randomization:

$$
M\left(x_{c}, s_{L}\right)=\min \left(1, \frac{F\left(\lambda_{c}\right)}{b}\right)
$$

The amount of liquidity is again given by,

$$
\varphi\left(x_{c}, \phi\right)=E\left[\lambda \mid \lambda<\lambda_{c}\right] F\left(\lambda_{c}\right) \min \left\{1, \frac{b}{F\left(\lambda_{c}\right)}\right\}
$$

\subsection{Investing Stage}

As before, $q^{R}\left(x_{c}, s\right)$ and $q\left(x_{c}, s\right)$ are related via $q^{R}=\frac{1-\theta q\left(x_{c}, s\right)}{1-\theta}$. Again, $q\left(x_{c}, s\right)$ is determined by market clearing condition. The demand for investment by s-entrepreneurs is:

$$
\begin{aligned}
& \underbrace{(1-\pi)\left(\varsigma^{s}(s) \frac{r}{q}-\left(1-\varsigma^{s}(s)\right) E_{\phi}[\lambda]\right) K}_{\text {Equity Demand S-types }} \\
= & \underbrace{\int\left(E_{\phi}[\lambda] B(\omega)-B(\omega)^{\prime}\right) d \omega+\pi \varphi\left(x_{c}, s\right) K}_{\text {Equity Supply - Fin. Firms }}+\underbrace{I^{s}(q)}_{\text {Demand - Investment }}
\end{aligned}
$$

This equation is similar to equation (17) except for the term $\int\left(E_{\phi}[\lambda] B(\omega)-B(\omega)^{\prime}\right) d \omega$ which captures the change in the financial systems reserves of equity. Financial firms will liquidate assets whenever they face needs to finance the loss in their activity. The supply of investment is on the other hand $q$ is given by

$$
I^{i}(q)=\pi\left[\varsigma^{i}(s) \frac{r+q \varphi\left(x_{c}, s\right)}{q^{R}}-\left(1-\varsigma^{i}(s)\right) \psi\left(x_{c}, s\right)\right] K
$$


As before, the aggregate incentive compatibility constraint 18 must be met. Again, $q=1$ if the constraint is slack and $q \geq 1$ otherwise. As with Proposition 8, $q$ is determined by the solution to a quadratic equation:

Proposition 14 (Market Clearing 2) Taking the liquidity functions $\varphi$ and $\psi$ and the marginal propensities to save $\varsigma^{s}$ and $\varsigma^{i}$ as given, there exists a unique $q$ that clears out the equity market and it is given by:

$$
q=\left\{\begin{array}{c}
1 \text { if } 1>q_{2}>q_{1} \\
q_{2} \text { if } q_{2}>1>q_{1} \\
1 \text { if otherwise }
\end{array}\right.
$$

where the terms $\left(q_{1}, q_{2}\right)$ are positive and given by:

$$
\begin{aligned}
& q_{1}=\frac{B^{2}}{2 A}-\frac{\sqrt{B^{2}-4 A C}}{2 A} \\
& q_{2}=\frac{B^{2}}{2 A}+\frac{\sqrt{B^{2}-4 A C}}{2 A}
\end{aligned}
$$

where

$$
\begin{gathered}
A=-\theta\left[\left(1-\varsigma^{s}\right)(1-\pi) E[\lambda]+\pi\left(\lambda\left(b-b^{\prime}\right)+\varphi\right)\right]+\pi H\left[\varphi \varsigma^{i}(1-\theta)+\theta \frac{\left(1-{ }^{i}\right) \psi}{(1-\theta)}\right] \\
B=r\left[\theta(1-\pi) \varsigma^{s}+H \varsigma^{i}(1-\theta)\right]-\pi H \frac{(1-\beta)}{(1-\theta)} \psi \\
C=-(1-\pi) \varsigma^{s} r
\end{gathered}
$$

and

$$
H=\left[\frac{(1-\theta)+(2-\theta) b^{\prime}}{\theta}\right]
$$

Proof. See appendix N.

Compared with Proposition 8, $q$ is now also determined by the change in reserves $b$ and $b /$. The change in reserves enters the supply of equity which is bought by s-entrepreneurs and therefore is also a determinant of equity prices. $b^{\prime}$ is determined endogenously by solving the financial firm's problem during the second stage:

Problem 3 (Financial Firms's problem) A financial $\omega$ has the following value function,

$$
W^{2}(B(\omega), u, s)=\max _{d, B(\omega)^{\prime}} d+\beta E\left[W^{1}\left(B(\omega)^{\prime}, s^{\prime}\right) \mid s\right]
$$

subject to it's budget constraint

$$
q B(\omega)^{\prime}+d=\left[q E_{\phi}[\lambda]+M u\right] B(\omega)
$$

and limited liability constraints

$$
\begin{gathered}
B(\omega)^{\prime} \geq 0 \\
d \geq 0
\end{gathered}
$$


Taking $q(s)$ and the value function $W^{1}\left(B(\omega)^{\prime}, s^{\prime}\right)$ as given, the solution to this problem determines the policy $b^{\prime}$. We conjecture that the value function is linear in the state $B(\omega)$ where the linear coefficient depends on $(u, s)$. This variables are, in turn, determined by equilibrium prices:

Proposition 15 The value function for the financial firm is linear in the individual state:

$$
W^{2}(B(\omega), u, s)=W^{2}(u, s) B(\omega)
$$

where the coefficient solves:

$$
W^{2}(u, s)=\max \left\{\beta E\left[W^{2}\left(u^{\prime}, s^{\prime}\right) \mid s\right], q(s) \lambda+M u\right\}
$$

Moreover,

$$
\begin{gathered}
B^{\prime}(\omega)=0 \text { if } \beta E\left[W^{2}\left(u\left(\phi^{\prime}, s\right), s\right) \mid s\right]<q \\
B^{\prime}(\omega)=\frac{(q \lambda+M u)}{q} \text { if } \beta E\left[W^{2}\left(u\left(\phi^{\prime}, s\right), s\right) \mid s\right]>q \\
B^{\prime}(\omega) \in\left[0, \max \left\{\frac{(q \lambda+u)}{q}, 0\right\}\right] \text { if } \beta E\left[W^{2}\left(u\left(\phi^{\prime}, s\right), s\right) \mid s\right]=q
\end{gathered}
$$

Proof. See appendix O.

In equilibrium, it will be the case that $\beta E\left[W^{2}\left(u\left(\phi^{\prime}, s\right), s\right) \mid s\right] \geq q \lambda+M u$. When this condition violated, no liquidity is provided at all. A financial firm deviating and waiting for one period would end up with the whole market. Thus this can not be an equilibrium. $b^{\prime}$ on the other hand determines $d$. Thus, when the condition is violated, we have, $\beta E\left[W^{2}\left(u\left(\phi^{\prime}, s\right), s\right) \mid s\right]=q$, and $B^{\prime}(\omega)$ is obtained by market clearing. Finally in states where $q=1$ no liquidity is provided and financial firms remain inactive.

Equilibria is computed using the following steps.

Algorithm 1 The equilibrium with financial risk capacity can be computed by,

1. Guess an initial value for $q, u$.

2. Compute the fixed point to the value function problem given by 31.

3. Use the solution to the value function to determine $(b /, q)$

(a) Guess $b^{\prime}=\frac{(q \lambda+M u)}{q} b$.

(b) Update $q$ from the quadratic formula and check if $\beta E\left[W^{2}\left(u\left(\phi^{\prime}, s\right), s\right) \mid s\right]>q$.

(c) If not, then, use $q=\beta E\left[W^{2}\left(u\left(\phi^{\prime}, s\right), s\right) \mid s\right]$, and compute bl from the quadratic formula.

4. Update $u, M$ using the solutions to $q, b$.

\subsection{Simulations with Financial Risk Capacity}

This section is not complete yet. 


\section{Conclusions}

The model presented in this paper describes how asymmetric information about capital quality endogenously determines the degree of liquidity in the equity market. Liquidity has real effects because investment is subject to limited enforcement. The main lessons from the analysis and simulations are:

1. As opposed to the RBC framework, recession can occur without changes in aggregate productivity.

2. Positive productivity shocks have a positive effect on liquidity and which reinforces the effect on investment.

3. The relation the dispersion of capital quality and liquidity mat be non-monotone but is positive under Assumption 2.

4. Under Assumption 2, an increase in the dispersion reduce liquidity.

5. The liquidity channel operates through a direct asymmetric information effect but also by distributing wealth toward investing entrepreneurs.

6. The model is consistent in generating a countercyclical equity premium, though the level of the premium is low.

7. A subsidy of $5 \%$ per financial transaction is required to shut down the liquidity channel.

The framework is extended to incorporate a financial sector that deals with asymmetric information but can't anticipate the the actual distribution of capital quality. By facing solvency constraints, the financial sectors's position determines the capacity to take risks when pooling equity contracts. We show that the liquidity channel was stronger and persistent through this mechanism.

We propose several directions to extend this paper. The first direction is to include a second asset as in KM. This asset can be paper money or a riskless bond. It would be interesting to know how asymmetric information is affected by this asset. Nevertheless, introducing a second asset introduces a technical difficulty when studying the behavior of the model globally. This difficulty is associated to a non-smoothness in the demand for this asset in states where $q=1$. One way to solve this difficulty is by introducing heterogeneity in the technical rate of transformation. This exercises is important to evaluate the effects of unexpected changes in it's supply would have real effects in this economy. It can provide a useful insight on how budget financing through taxes and debt instruments may have effects on liquidity. Finally, introducing an additional asset may be useful to understand the way bubbles can effect liquidity and why they may form.

Studying financial risk capacity in more detail may be an alternative promising direction for future research. In reality, important shocks to the financial system occur unexpectedly and quickly. In terms of our model, this suggests that shocks to financial system's build on systematic mistakes about the distribution of capital quality. It may be worthy to explore if heterogeneous information on the side of financial firms may cause systematic mistakes and let the financial system suddenly aware of it. Perhaps the right place to begin looking is in frameworks such as Lorenzoni [2009] or Angeletos and La'O [2009]. On the other hand, normative analysis in the context of financial risk capacity may become useful for policy purposes.

Finally and most importantly, we would like to have empirical evidence on asymmetric information in financial markets. These tasks are left for the future. 


\section{References}

G. A. Akerlof. The market for 'lemons': Quality uncertainty and the market mechanism. Quarterly Journal of Economics, 84(3):488-500, 1970. 6

G.-M. Angeletos. Uninusured idiosyncratic investment risk. Review of Economic Dynamics, 10: $1-30,2007.4,5,15,17,21$

G.-M. Angeletos and J. La'O. Sentiments. Unpublished Manuscript, 2009. 46

M. Bagnoli and T. Bergstrom. Log-concave probability and its applications. Economic Theory, 26 (2):445-469, August 2005. 6, 58

S. Bigio. Financial risk capacity. Mimeo New York University, 2010a. 2

S. Bigio. A survey on liquidity. Mimeo New York University, 2010b. 3

A. Bisin and P. Gottardi. Competitive equilibria with asymmetric information. Journal of Economic Theory, 87:1-48, August 1999. ISSN 0022-0531. 5, 12

M. K. Brunnermeier. Deciphering the liquidity and credit crunch 20072008. Journal of Economic Perspectives, 23(1):77-100, 2009. 2

C. Campanale, R. Castro, and G. L. Clementi. Asset pricing in a production economy with chewdekel preferences. The Review of Economic Studies, forthcoming, 2009. 21

V. Curdia and M. Woodford. Conventional and unconventional monetary policy. 2009. 3

D. W. Diamond and P. H. Dybvig. Bank runs, deposit insurance, and liquidity. Journal of Political Economy, 91(3):401-419, 1983. 3

A. L. Eisfeldt. Endogenous liquidity in asset markets. The Journal of Finance, 59(1):1-30, February 2004. 3

L. G. Epstein and S. E. Zin. Substitution, risk aversion, and the temporal behavior of consumption and asset returns: A theoretical framework. Econometrica, 54(4), July 1989. 5

M. Gertler and P. Karadi. A model of unconventional monetary policy. 2009. 3

M. Gertler and N. Kiyotaki. 2010. 3

B. Holmstrom and J. Tirole. Private and public supply of liquidity. Journal of Political Economy, 106(1), August 1998. 3

U. J. Jermann. Asset pricing in production economies. Journal of Monetary Economics, 41:257-275, 1998. 34

N. Kiyotaki and J. Moore. Liquidity, business cycles, and monetary policy. Unpublished Manuscript, 2008. 3

P. Kurlat. Lemons, market shutdowns and learning. MIT - Working Paper, 2009. 3, 6, 8, 14, 15, 31

R. Lagos and R. Wright. Unified framework for monetary theory and policy analysis. Journal of Political Economy, 113(3):463-484, 2005. 4 
G. Lorenzoni. A theory of demand shocks. American Economic Review, forthcoming, 2009. 46

G. Lorenzoni and K. Walentin. Financial frictions, investment and tobin's q. Review of Economics Studies, Forthcoming 2009. 25

A. F. Marco Del Negro, Gauti Eggertsson and N. Kiyotaki. The great escape? a quantitative evaluation of the feds non-standard policies. 2010. 3

T. J. Moskowitz and A. Vissing-Jørgensen. The returns to entrepreneurial investment: A private equity premium puzzle? The American Economic Review, 2002. 21

E. C. Prescott and R. M. Townsend. Pareto optima and competitive equilibria with adverse selection and moral hazard pareto optima and competitive equilibria with adverse selection and moral hazard. Econometrica, 52(1):21-45, Januaryt 1984. 5, 12

G. Primiceri, A. Justiniano, and A. Tambalotti. Investment shocks and the relative price of investment. Unpublished Manuscript. 6

C. M. Reinhart and K. S. Rogoff. This time is different: A panoramic view of eight centuries of financial crises. Working Paper - Harvard University. 2

T. J. Sargent. Tobin's q and the rate of investment in general equilibrium. The American Economic Review, 12(1):107-154, 1980. 17

J. E. Stiglitz and A. Weiss. Credit rationing in markets with imperfect information. American Economic Review, 71(3):393-410, 1981. 3

T. D. Tallarini. Risk-sensitive real business cycles. Journal of Monetary Economics, 45:507-532, 2000. 21,34

H. Uhlig. A law of large numbers for large economies. Economic Theory, 8(1):41-50, February 1996. ISSN 0938-2259 (Print) 1432-0479 (Online). 49

A. Vissing-Jørgensen and O. P. Attanasio. Stock-market participation, intertemporal substitution, and risk-aversion. The American Economic Review, 93(2):383-391, May 2003. 21

C. Wilson. The nature of equilibrium in markets with adverse selection. The Bell Journal of Economics, 11(1):108-130, Spring 1980. 13, 55, 56

C. A. Wilson. Equilibrium and adverse selection. The American Economic Review, 69(2):313-317, May 1979. 13, 55, 56 


\section{A Technical Lemma on $\lambda_{t}(z)$}

This section establishes a result that concerns the behavior of random variables that are integrals of function of $\lambda_{t}(z)$ with respect to $z \in[0,1]$. These sort of integrals will appear entrepreneurs budget constraint. This section establishes that these integrals are well defined and that the law of large numbers can be invoked to compute them. The reader may wish to skip this section.

$\lambda_{t}(z)$ defines a random variable in the space of functions in $[0,1]$. Let $\mathbf{B}([0,1])$ be the Borel $\sigma$-algebra of the unit interval and $P_{F_{\phi}}$ be the probability measure corresponding to the cumulative distribution function $F_{\phi}$. By assumption $\lambda_{t}(z) \in L^{2}\left\{[0,1], \mathbf{B}[0,1], P_{F_{\phi}}\right\}$, since it is a random variable with finite variance. Thus, $\lambda_{t}(z)$ satisfies the conditions in Theorem 2 of Uhlig [1996] and we have that,

$$
\int_{0}^{1} \lambda_{t}(z) d z \rightarrow p . \mu_{\phi}
$$

where the integral is defined in the Riemann sense. Analogously,

$$
\operatorname{Pr}\left[\lambda_{t}(z)<\bar{\lambda}\right] \rightarrow p \int 1_{\bar{\lambda}} d z=F_{\phi}(\bar{\lambda})
$$

where $\rightarrow p$ refers to convergence in probability. Extending this result, we establish, Lemma 1 in the document:

Lemma 3 Let $H:[0,1] \rightarrow R$ be a bounded measurable function, and let $e(z) \in L^{2}([0,1])$. Then for a given realization of $s_{t}$, the following holds,

$$
\int H(\lambda(z)) e(z) d z \rightarrow{ }_{p} E\left[H(\lambda) \mid s_{t}\right] \int e(z) d z
$$

In the paper a random integral term like $E_{\phi}\left[\int H(\lambda(z)) e(z) d z\right]$ is treated as the constant $E_{\phi}[H(\lambda)] \int e(z) d z$ it converges to.

\section{A.1 Proof of Lemma 1}

Let $\varphi_{n}$ be a partition of $\mathrm{n}$ intervals in unit interval. Let $\left\{i_{j}\right\}_{j=0}^{n}$ denote the endpoints of each element of the partition with $0=i_{0}<i_{1}<\ldots<i_{n}=1$, and $\psi_{j} \in\left[i_{j-1}, i_{j}\right], j \in\{1,2, . ., n\}$, be interior points at each interval. Let $m_{n}=\max _{\left[i_{j-1}, i_{j}\right] \in \varphi_{n}}\left[i_{j-1}, i_{j}\right]$. Then, for a given realization of $\lambda(z)$, a sequence of random variables $\left\{S_{n}\right\}$, may be defined by

$$
S_{n}=\sum_{i=0}^{n} H\left(\lambda\left(\psi_{j}\right)\right) e\left(\psi_{j}\right)\left[i_{j-1}, i_{j}\right] \text { and }\left[i_{j-1}, i_{j}\right] \in \varphi_{n}
$$

One needs to show that the limit of this sequence of random variables converges in $L_{2}$.

where in the second line $o(n)$ is the difference between $E_{\phi}[H(\lambda)] \int e(z) d z$ and it's $n-t h$ approximation, the third line collects terms, the fourth line uses the independence of $\lambda\left(\psi_{j}\right)$, the fifth line hinges on $H$ being bounded and the definition of $m_{n}$. Finally, the fact that $\int_{0}^{1} e(z)^{2} d z$ is finite since, $e(z) \in L^{2}$. Finally, convergence in probability follows from Chebyshev's inequality. See Durrett, Lemma (5.3), chapter 1. 


\section{B Trades at the Clean-Up Stage}

At the final stage investment projects are realized and agents inherit an equity portfolio from the previous period. The i-entrepreneurs portfolio may be concentrated in his own firm. When outside equity is fully diversified, idiosyncratic risk is minimized. Thus, entrepreneurs have incentives to trade at this stage in order to diversify risk. This section shows that the portfolio weights on other entrepreneurs firms will be the same for all entrepreneurs. This way, portfolios and wealth distributions are not state variables in the model.

The goal of this section is to show that if the value function at the beginning of each period is an increasing and homogeneous function $V$ of some definition of wealth $w_{t+1} \in \mathbb{R}_{+}$, then the value function can be written only in term of equity $n_{t}$. Wealth in turn is a function of $\left(e_{t+1}^{-}(\cdot, z), e_{t+1}^{+}(\cdot, z), k(z)\right)$ and an arbitrary function $\chi: R_{+} \times R_{+} \rightarrow R_{+}$such that

$$
w_{t}\left(s_{t}\right) \equiv r_{t} n_{t}+\int \chi_{t}\left(\lambda(\tilde{z}), s_{t+1}\right) \lambda(\tilde{z}) e_{t}^{+}(\tilde{z}, z) d \tilde{z}+\chi_{t}\left(\lambda(z), s_{t+1}\right) \lambda(z)\left(k(z)-\int e_{t}^{-}(\tilde{z}, z) d \tilde{z}\right)
$$

$\chi$ is an object that we will realte to endogenous variables of the model.

To show this result first define the cumulative distribution function $G(\hat{k}) \equiv \mu(\{z \mid k(z) \leq \hat{k}\})$, where $\mu$ is the population measure. Let $g(\hat{k})$ be the density associated with $G(\hat{k})$. where $\chi\left(\cdot, s_{t}\right)$ : $[0,1] \rightarrow \mathbb{R}_{+}$are bounded, continuous and increasing function of $\lambda$. Hence the entrepreneur's problem is,

Problem 4 (Optimal Portfolio)

$$
W\left(n_{t+1}, e_{3, t}^{+}, e_{3, t}^{-}, s_{t}\right)=\max _{\Delta e_{3, t}^{+}(\cdot, z), \Delta e_{3, t}^{-}(\cdot, z)} E_{t}\left[V\left(w_{t+1}\right) \mid s_{t}\right]
$$

subject to:

$$
\begin{gathered}
q_{t}\left[\int_{0}^{1} \Delta e_{3, t}^{+}(\tilde{z}, z)+\Delta e_{3, t}^{-}(\tilde{z}, z) d \tilde{z}\right]=0 \\
\int_{0}^{1} \Delta e_{3, t}^{+}(\tilde{z}, z) \leq k_{t}(z)-\int_{0}^{1} e_{3, t}^{-}(\tilde{z}, z) \\
e_{t+1}^{+}(\tilde{z}, z)=\Delta e_{3, t}^{+}(\tilde{z}, z)+e_{3, t}^{+}(\tilde{z}, z)
\end{gathered}
$$

and

$$
e_{t+1}^{-}(\tilde{z}, z)=\Delta e_{3, t}^{-}(\tilde{z}, z)+e_{3, t}^{-}(\tilde{z}, z)
$$

Because wealth takes the particular form of (32) and the value function is concave, all portfolios at the end of the period will be identical. In particular, they have an analytic solution:

Proposition 16 (Homogeneous Portfolio) The solution to Problem 4, is given by

$$
\begin{gathered}
\Delta e_{3, t}^{+}(\tilde{z}, z)=g(\tilde{z}) n_{t+1}(z)-e_{3, t}^{+}(\tilde{z}, z) \\
\Delta e_{3, t}^{-}(\tilde{z}, z)=k(z)-\int e_{t+1}^{-}(\tilde{z}, z) d \tilde{z}
\end{gathered}
$$

so

$$
e_{t+1}^{+}(\tilde{z}, z)=g(\tilde{z}) n(z) \text { for } \forall \tilde{z}, z
$$




$$
\int e_{t+1}^{-}(\tilde{z}, z) d \tilde{z}=k(z)
$$

This allocation is feasible at the aggregate level and Pareto efficient. Moreover, given an aggregate state $s_{t}$ the value function of this problem is an increasing function of equity $W\left(n_{t+1}, s_{t}\right)$ only.

Proof. See appendix B.1.

Given this proposition, wealth defined by (32) collapses to,

$$
w_{t+1}=\left(r_{t}+\int \chi_{t}\left(\lambda(\tilde{z}), s_{t}\right) \lambda(\tilde{z}) g(\tilde{z}) d \tilde{z}\right) n_{t}
$$

Hence, wealth is a linear function of equity holdings and independent of the original portfolio composition. In fact, when computing the expectation in (33), by homogeneity of $V$, and using (38), we obtain,

$$
W\left(n_{t+1}, s_{t}\right)=E_{t}\left[V\left(w_{t+1}\right) \mid s_{t}\right]=n_{t+1}^{p} E_{t}\left[V\left(r_{t}+\int \chi_{t}\left(\lambda(\tilde{z}), s_{t}\right) \lambda(\tilde{z}) g(\tilde{z}) d \tilde{z}\right) \mid s_{t}\right]
$$

for some power $p$. Thus, given the structure described here, the agents value function depends only on its equity holdings $n_{t}$ and no other idiosyncratic component. We have therfore shown that,

Proposition 17 (Net Equity) The value function at stage 3 is an increasing function of net equity only. .

In addition, with Proposition 16 we can aggregate equity and show that it is equal to the aggregate capital stock (the derivation is found in appendix B.2).

Remark 3. $\int_{0}^{1} n_{t}(z) d z=K_{t}$.

\section{B.1 Proof of Proposition 16}

Wealth at period $t+1$ is given by (32). Let $w^{o}$ be the wealth level induced by the optimal policies (34) and (35), and let $\tilde{w}$ be any alternative policy. By concavity of $V$, we that

$$
W\left(w^{o}\right)-V(\tilde{w}) \geq V^{\prime}\left(w^{o}\right)\left(w^{o}-\tilde{w}\right)
$$

taking expectations conditional on $s_{t+1}$ on both sides yields,

$$
\begin{aligned}
W\left(w^{o}\right)-W(\tilde{w}) & \geq E\left[V^{\prime}\left(w^{o}\right) \mid s_{t+1}\right] E_{t}\left[\left(w^{o}-\tilde{w}\right) \mid s_{t+1}\right] \\
& =E_{t}\left[V^{\prime}\left(w^{o}\right) \mid s_{t+1}\right] w^{o}\left(s_{t+1}\right)-E_{t}\left[V^{\prime}\left(w^{o}\right) \mid s_{t+1}\right] E_{t}\left[\tilde{w} \mid s_{t+1}\right] \\
& =E_{t}\left[V^{\prime}\left(w^{o}\right) \mid s_{t+1}\right] w^{o}\left(s_{t+1}\right) \\
& \geq 0
\end{aligned}
$$

where the first inequality follows from noticing that $w^{o}$ is constant conditional on $s_{t+1}$, (by Lemma $1, \int \chi_{t+1}\left(\lambda(\tilde{z}), s_{t+1}\right) \lambda(\tilde{z}) e_{t+1}^{+}(\tilde{z}, z) d \tilde{z}$ is constant) and the third line follows by independence and Lemma 1. Integration with respect to $\mathrm{s}_{t+1}$, yields the desired result. These policies are clearly feasible to the entrepreneur. Since, 


$$
\begin{aligned}
& k_{t}(z)-\int_{0}^{1} e_{3, t}^{-}(\tilde{z}, z) d \tilde{z}+k_{t}(z)+\int_{0}^{1} e_{3, t}^{+}(\tilde{z}, z) d \tilde{z} \\
= & k_{t}(z)-\int_{0}^{1} e_{3, t}^{-}(\tilde{z}, z) d \tilde{z}+\int_{0}^{1} e_{3, t}^{+}(\tilde{z}, z) d \tilde{z}+\int_{0}^{1} \Delta e_{3, t}^{-}(\tilde{z}, z) d \tilde{z}-\int_{0}^{1} \Delta e_{3, t}^{-}(\tilde{z}, z) d \tilde{z} \\
= & \int_{0}^{1} e_{3, t}^{+}(\tilde{z}, z) d \tilde{z}+\int_{0}^{1} \Delta e_{3, t}^{-}(\tilde{z}, z) d \tilde{z}
\end{aligned}
$$

so by plugging (8) and then (34) leads to

$$
\begin{aligned}
& \int_{0}^{1} e_{3, t}^{+}(\tilde{z}, z) d \tilde{z}-\int_{0}^{1} \Delta e_{3, t}^{+}(\tilde{z}, z) d \tilde{z} \\
= & \int_{0}^{1} g(\tilde{z}) n_{t+1}(z) d \tilde{z} \\
= & n_{t+1}(z)
\end{aligned}
$$

so the policy is feasible. To show that the policy is feasible at the aggregate level, integrate the above expression to obtain,

$$
\begin{aligned}
\int n_{t+1}(z) d z & =\int_{0}^{1} \int_{0}^{1} g(\tilde{z}) n_{t+1}(z) d \tilde{z} d z \\
& =\int_{0}^{1} n_{t+1}(z) d z \int_{0}^{1} g(\tilde{z}) d \tilde{z} \\
& =\int_{0}^{1} n_{t+1}(z) d z \\
& =K_{t+1}
\end{aligned}
$$

Thus, the policy is Pareto optimal and feasible at aggregate and individual levels. Finally, observe that wealth evaluated at the optimal policies is

$$
\begin{aligned}
w_{t+1} & =r_{t} n_{t}+\int \chi_{t}\left(\lambda(\tilde{z}), s_{t+1}\right) \lambda(\tilde{z}) e_{t}^{+}(\tilde{z}, z) d \tilde{z} \\
& =\left(r_{t}+\int \chi_{t}\left(\lambda(\tilde{z}), s_{t+1}\right) \lambda(\tilde{z}) g(\tilde{z}) d \tilde{z}\right) n_{t}
\end{aligned}
$$

which is a function independent of the portfolio.

\section{B.2 Proof of Corollary 3}

Using direct identities we have 


$$
\begin{aligned}
\int_{0}^{1} n_{t}(z) d z & =\int_{0}^{1} \int_{0}^{1} e_{t-1}^{+}(\tilde{z}, z) d \tilde{z} d z \\
& =\int_{0}^{1} \int_{0}^{1} e_{t-1}^{-}(\tilde{z}, z) d \tilde{z} d z \\
& =\int_{0}^{1} \int_{0}^{1} e_{t-1}^{-}(\tilde{z}, z) d z d \tilde{z} \\
& =\int_{0}^{1} k(\tilde{z}) d \tilde{z} \\
& =K_{t}
\end{aligned}
$$

\section{Investment opportunities and Optimal Financing at Stage 3 (Investing)}

Given Proposition (16), at the second stage, the entrepreneur's problem consists on choosing consumption, a downpayment for investment projects and purchases of outside equity. When the value function at stage 4 takes the form of (39) then the entrepreneurs problem is,

Problem 5 (Stage 2 Problem)

$$
\max _{c_{t}, \Delta e_{2, t}^{+}(\cdot, z), i_{t}^{d}} U\left(c_{t}\right)+\beta W\left(n_{t+1}(z), s_{t}\right)
$$

subject to

$$
\begin{aligned}
c_{t}+i_{t}^{d}+q_{t} \int_{0}^{1} \Delta e_{2, t}^{+}(\tilde{z}, z) d \tilde{z}=r_{t} n_{t}(z)+x_{t}(z) \\
i_{t}^{d}+i_{t}^{f}=i_{t}, i_{t}^{i}+i_{t}^{s}=i_{t}, q_{t} i_{t}^{s}=i_{t}^{f} \\
i_{t}^{s} \leq \theta i_{t} \\
n_{t+1}(z)=\lambda k_{t}(z)+i_{t}^{i}+\int_{0}^{1}\left(e_{3, t}^{+}(\tilde{z}, z)-e_{3, t}^{-}(\tilde{z}, z)\right) d \tilde{z}
\end{aligned}
$$

We return to this problem in Section ?? and find a value function $W\left(n_{t+1}, s_{t}\right)$ entirely consistent with the preference specification. For the moment, we analyze the problem by breaking it up into pieces. Here, we analyze the optimal financing decision when consumption is taken as given. First, for a scale of downpayment, $i_{t}^{d}$ the entrepreneur will choose a financing structure to maximize his equity holdings in future periods subject to incentive compatibility requirement by other entrepreneurs. This problem is,

Problem 6 (Optimal Financing)

$$
\max _{i_{t}^{s}>0} i_{t}^{i}
$$

taking $i_{t}^{d}>0$ as given and subject to:

$$
\begin{aligned}
i_{t}^{d}+i_{t}^{f} & =i_{t}, i_{t}^{i}+i_{t}^{s}=i_{t}, q_{t} i_{t}^{s}=i_{t}^{f} \\
i_{t}^{s} & \leq \theta i_{t}
\end{aligned}
$$


Substituting all the constraints, the problem is written in terms of $i_{t}^{d}$ and $i_{t}^{s}$ only:

Problem 7 (Optimal Financing Dual)

$$
\max _{i_{t}^{s}} i_{t}^{d}+\left(q_{t}-1\right) i_{t}^{s}
$$

taking $i_{t}^{d}$ as given and subject to:

$$
i_{t}^{s} \leq \theta i_{t}^{d}+\theta q_{t} i_{t}^{s}
$$

For every project it runs, the investing entrepreneur increases his stock of equity $i_{t}^{d}+\left(q_{t}-1\right) i_{t}^{s}$, which is the sum of the down payment plus the arbitrage opportunity obtained by issuing equity. Outside funding is limited by the incentive compatibility constraint. As $q_{t}$ is lower, the constraint on external funding is tighter because the investing entrepreneur's stake on the project is lower. It will be shown that $q_{t} \in\left[1, \frac{1}{\theta}\right)$.. Note that for every unit of investment $i_{t}$, the i-entrepreneurs finances $\left(1-\theta q_{t}\right)$ units and owns the fraction $(1-\theta)$. This defines a cost of generating equity for the investing entrepreneur,

$$
q_{t}^{R}=\left[\frac{\left(1-\theta q_{t}\right)}{(1-\theta)}\right] \leq 1 \text { with equality if } q_{t}=1
$$

The difference between $q_{t}^{R}$ and $q_{t}$ is a wedge between the cost of purchasing outside equity and the cost of generating inside equity. Thus, we have Proposition 2 in the text,

Proposition 18 If $q_{t}>1$, the solution to Problem 5 is

$$
\Delta e_{2, t}^{+}(\tilde{z}, z)=0 \text { and } i_{t}^{s}=\theta i_{t}
$$

Proof. See appendix C.1.

This proposition states that without loss of generality one can assume that no outside equity is purchased by i-entrepreneurs at this stage. Using the definition of equity (2) and the result in Proposition 18, allows us to define the evolution of equity as:

$$
n_{t+1}(z)=\lambda(z) k_{t}(z)+\left(q_{t}^{R}\right)^{-1} i_{t}^{d}+\int_{0}^{1}\left(e_{2, t}^{+}(\tilde{z}, z)-e_{2, t}^{-}(\tilde{z}, z)\right) d \tilde{z}
$$

Clearing out $i_{t}^{d}$ and replacing it into (5), one can rewrite the budget constraint by using (18) into:

$$
c_{t}+q_{t}^{R}\left(n_{t+1}(z)-\lambda(z) k_{t}(z)-\int_{0}^{1}\left(e_{2, t}^{+}(\tilde{z}, z)-e_{2, t}^{-}(\tilde{z}, z)\right) d \tilde{z}\right)=r_{t} n_{t}(z)+x_{t}(z)
$$

and rearranging terms we obtain an alternative budget constraint at the second stage becomes,

$$
c_{t}+q_{t}^{R} n_{t+1}(z)=r_{t} n_{t}(z)+x_{t}(z)+q_{t}^{R}\left(\lambda(z) k_{t}(z)+\int_{0}^{1}\left(e_{2, t}^{+}(\tilde{z}, z)-e_{2, t}^{-}(\tilde{z}, z)\right) d \tilde{z}\right)
$$

Before proceeding to the characterization at the signing stage, it may be instructive to discuss what is the role played by the signed amounts $x_{t}(z)$. 


\section{C.1 Proof of Proposition 18}

It is clear that if $q_{t}>1, i_{t}^{s}=\theta i_{t}$ is the solution to 7 . Substituting this result, the cost of increasing equity is $q_{t}^{R}<q_{t}$. Thus, $\Delta e_{2, t}^{+}(\tilde{z}, z)>0$ cannot be a solution to 5 .

\section{Equity Market at Stage 2 (Signing)}

At the beginning of the signing stage entrepreneurs write contracts with financial firms. Here, contracts are assumed to be anonymous and non-exclusive but as pointed out by Wilson [1979] and Wilson [1980] the equilibrium outcomes in markets with private information are sensitive to the market institutions assumed.

Financial firms offer a contracts which specify an amount $x_{c} \geq 0$ in exchange for $e_{c} \geq 0$ equity and a recommendation of whose equity to sell. Upon signing the contract, $x_{c}$ goods will be delivered to the i-entrepreneur in the following stage. Entrepreneurs are allowed to purchase arbitrary amounts of this contracts. Since the original issuer of equity is not observed by financial firms, the contract is not conditioned on the portfolio of sold equity, $\Delta e_{t, 1}^{+}(\cdot, z)$. The size of the sold portfolio must equal the amount of equity specified by all contracts signed by the entrepreneur. Because entrepreneurs only care about the sold portfolio it follows without loss in generality that each portfolio is identical.

Under non-exclusive contracts, offered contracts impose externalities on other contracts which complicates the notion of incentive compatibility so a competitive equilibrium is defined directly. To do so, we restrict the analysis to symmetric equilibria. In particular, the equity holdings $e_{1, t}^{+}(\cdot, z)$ are proportional to the value of each entrepreneurs equity $n(z)$.

Definition (Competitive Symmetric Signing Equilibrium). Assume that the portfolio compositions $e_{1, t}^{+}(\cdot, z)$ are proportional to $n(z), \forall z$ and take as given a price of equity $q_{t}$. A symmetric competitive equilibrium at the signing stage is a set of contracts $C$ and together with a portfolio $\Delta e_{t+1}^{+}(\tilde{z}, z)=$ $\Delta e_{t+1}^{+}(\tilde{z}) n(z) \forall z$, and a measure $\mu_{c}$ defined over $C$ such that the following conditions are satisfied $\forall c, z$ :

\section{Profitability - Free Entry:}

$$
x_{c}-q_{t} \int_{0}^{1} \lambda(\tilde{z}) \Delta e_{t+1}^{+}(\tilde{z}, z) d \tilde{z}=0
$$

for each $c \in C$.

2. Optimality: the support of $\mu_{c}$ and the optimal portfolio $\Delta e_{t+1}^{+}(\tilde{z})$ is given by the set of contracts that solve,

$$
\max _{\mu_{c} \in M(C), \Delta e_{t+1}^{+}(\tilde{z}, z)} \int_{C} x_{c}-q_{t}^{R}\left(\int_{0}^{1} \lambda(\tilde{z}) \Delta e_{t+1}^{+}(\tilde{z}) d \tilde{z}\right) \mu_{c}(d c)
$$

where $M(C)$ is the set of measures over the set of possible contracts and $\forall z$, it is the case that

$$
e_{t+1}^{+}(\tilde{z}, z) d \tilde{z}-n(z) \int_{C} \Delta e_{t+1}^{+}(\tilde{z}) \mu_{c}(d c) \geq 0, \forall \tilde{z}
$$


The first condition, (43), is a zero profit condition. The second, (44), requires that entrepreneurs choose a set of contracts optimally together subject to the quantity requirement of each contract. The objective is consistent with the definition of wealth in the second stage (the right hand side of 42). The signing equilibrium contract is always a pooling equilibrium. We have Proposition 3 presented in the text:

Proposition 19 (Signed Contracts) Every Competitive Symmetric Signing Equilibrium is characterized by a single pooling contract.

For any $q_{t} \in\left(1, \frac{1}{\theta}\right]$ such contracts are characterized by the pair $\left(x_{*}, \lambda_{*}\right)$ that solve the following:

$$
\lambda_{*}=\min \left\{1, \frac{x_{*}}{q_{t}^{R}}\right\}
$$

and

$$
x_{*}=q_{t} E_{\phi}\left[\lambda(\tilde{z}) \mid \lambda(\tilde{z}) \leq \lambda_{c}\right]
$$

where $e_{*}$ is given by the right hand side of (10) and $\mu\left(x_{*}, \lambda_{*}\right)=1$.

If $q_{t}=1$, then wlog,

$$
x_{*}=0 \text { and } \lambda_{*}=\lambda_{C}
$$

Moreover, under this equilibrium contract, the agents optimal policy imply that,

$$
x_{t}=x_{*} F_{\phi}\left(\lambda_{c}\right) n_{t}(z)
$$

and

$$
\begin{aligned}
\Delta e_{t+1}^{+}(\tilde{z}, z) & =-e_{t+1}^{+}(\tilde{z}, z) \text { if } \lambda(\tilde{z}) \leq \lambda_{*} \text { and } \\
\Delta e_{t+1}^{+}(\tilde{z}, z) & =0 \text { otherwise }
\end{aligned}
$$

Proof. See appendix D.1.

This proposition states that any solution to the equations described above, characterizes a unique contract for each equilibrium. Yet, as with the standard lemons market, multiple solutions may solve this pair of equations (Wilson [1979] and Wilson [1980]). Hence, in spite of having a unique contract in each equilibrium, the equilibrium itself may not be unique. Within an equilibrium contracts pool different equity classes together. There is no way to screen agents since quantities are unobserved by financial firms and contracts are non-exclusive. Within an equilibrium, the contract is unique because agents will approach the contract that grants them the highest amount of liquid assets. Rather than refining the notion of equilibria, we chose to impose assumption 2 which restricts attention to a set of the density families $f_{\phi}$ such that equilibria a uniqueness result holds:

Proposition 20 (Equilibrium Liquidity ) Under assumption 2, for any $q_{t} \in\left(1, \frac{1}{\theta}\right]$, there exists a unique $\left(x^{*}, \lambda^{*}\right)$ satisfying (9) and (10). In addition, the solutions $\left(x^{*}, \lambda^{*}\right)$ are increasing functions $\phi$.

Proof. See appendix D.2. 


\section{D.1 Proof of Proposition 3}

\section{Show that savers are segmented if their type is observed.}

First, we show that there cannot be two different contracts in equilibrium. Suppose that there are at least two different equilibrium contracts $(x, c)$ and $\left(x^{\prime}, c^{\prime}\right)$ then by (43) if $x>x^{\prime}$ then $c>c$. Suppose that $\left(x^{\prime}, c^{\prime}\right)$ has a positive measure. Then holding fixed $\Delta e_{t+1}^{+}(\tilde{z})$, the agent can improve its problem by choosing and alternative measure where the mass assigned to $\left(x^{\prime}, c^{\prime}\right)$ is now assigned to $(x, c)$. If in turn, $\left(x^{\prime}, c^{\prime}\right)$ has zero measure but belongs to some sufficiently small neighborhood $B_{\varepsilon}\left(x^{\prime}\right)$ with positive mass and where $(x, c) \notin B_{\varepsilon}\left(x^{\prime}\right)$, then, again there is an alternative measure where the mass of $B_{\varepsilon}\left(x^{\prime}\right)$ is assigned to $(x, c)$, such that it maximizes the agents problem. Finally, note that if it is not the case that there exists another contract with positive measure, or that lives in a set with positive measure that doesn't contain $(x, c)$, then $(x, c)$ is a the unique contract traded with positive measure.

Since all the mass in $\mu_{c}$ is assigned to the same contract, (44) is equivalent to,

$$
\max _{\Delta e_{t+1}^{+}(\tilde{z}, z)} x_{c}-q_{t}^{R} \int_{0}^{1} \lambda(\tilde{z}) \Delta e_{t+1}^{+}(\tilde{z}) d \tilde{z}
$$

subject to,

$$
e_{t+1}^{+}(\tilde{z}, z) d \tilde{z}-n(z) \mu_{c} \Delta e_{t+1}^{+}(\tilde{z}) \geq 0
$$

The solution to the entrepreneurs problem is in turn, given by,

$$
\begin{aligned}
\Delta e_{t+1}^{+}(\tilde{z})= & \frac{e_{t+1}^{+}(\tilde{z}, z) d \tilde{z}}{n(z) \mu_{c}} \text { if } x_{c} \geq q_{t}^{R} \lambda(\tilde{z}) \\
& \text { and } \\
= & 0 \text { otherwise }
\end{aligned}
$$

Therefore, let $\lambda_{c}=\min \left\{1, \frac{x_{c}}{q_{t}^{R}}\right\}$. Therefore, substituting this conditions into the (43) condition yields,

$$
\begin{aligned}
0 & =x_{c}-q_{t} \int_{0}^{1} \lambda(\tilde{z}) \Delta e_{t+1}^{+}(\tilde{z}) d \tilde{z} \\
& =x_{c}-\frac{q_{t}}{\mu_{c}} \int_{0}^{1} I_{\left[\lambda(\tilde{z})<\lambda^{c}\right]} \lambda(\tilde{z}) \Delta e_{t+1}^{+}(\tilde{z}) d \tilde{z}
\end{aligned}
$$

Case 1: $q_{t}=1$, the only possible solution is $x_{c}=0, \lambda_{c}=\lambda_{L}$.

Case 2: $q_{t}>1$, then observe that the number of contracts signed by each agent $\mu_{c}$, must equal $F_{\phi}\left(\lambda_{c}\right)$, the fraction of equity with quality below $\lambda_{c}$. If this quantity is larger, this means that some equity of quality higher than $\lambda_{c}$ was sold. In turn, if this quantity is lower there is some equity that was not sold. Multiplying both sides by $F_{\phi}\left(\lambda_{c}\right)$ and rearranging terms yields,

$$
x_{c}=q_{t} \frac{E_{\phi}\left[\lambda(\tilde{z}) \mid \lambda(\tilde{z}) \leq \lambda_{c}\right]}{F_{\phi}\left(\lambda_{c}\right)}
$$

There are potentially multiple solutions to (9) and (10). Nevertheless, by relying on 2, Proposition 20 insures that the solution must is unique and always exists. 


\section{D.2 Proof of Proposition 20}

Replacing (10) into $\frac{x_{c}}{q_{t}^{R}}$ one obtains,

$$
\lambda_{c}=\min \left\{1, \frac{q_{t}}{q_{t}^{R}} \frac{E_{\phi}\left[\lambda(\tilde{z}) \mid \lambda(\tilde{z}) \leq \lambda_{c}\right]}{F_{\phi}\left(\lambda_{c}\right)}\right\}
$$

Inside the second term in the brackets we find,

$$
\begin{aligned}
\frac{E_{\phi}\left[\lambda(\tilde{z}) \mid \lambda(\tilde{z}) \leq \lambda_{c}\right]}{F_{\phi}\left(\lambda_{c}\right)} & =\frac{\int_{\lambda_{c}}^{\lambda_{c}} \lambda f_{\phi}(\lambda) d \lambda}{F_{\phi}\left(\lambda_{c}\right)} \\
& =\lambda_{c}-\frac{\int_{\lambda_{c}}^{\lambda_{c}} F_{\phi}(\lambda) d \lambda}{F_{\phi}\left(\lambda_{c}\right)} \\
& =\lambda_{c}-\frac{G_{\phi}\left(\lambda_{c}\right)}{G_{\phi}^{\prime}\left(\lambda_{c}\right)}
\end{aligned}
$$

where the first inequality uses Lemma 1, the second follows from integration by parts and the last follows from the definition in part (iii) in Assumption 2. Ignoring the constraint that $\lambda^{*}<$ 1 , so without the constraint, the solution solves,

$$
\frac{G_{\phi}\left(\lambda_{c}\right)}{G_{\phi}^{\prime}\left(\lambda_{c}\right)}=\frac{q_{t}^{R}}{q_{t}}\left(\frac{q_{t}}{q_{t}^{R}}-1\right) \lambda_{c}
$$

The solution, if it exists, is unique since under condition (ii) in Assumption 2, this ensures that $\frac{G_{\phi}\left(\lambda_{c}\right)}{G_{\phi}^{\prime}\left(\lambda_{c}\right)}$ is decreasing. See, Bagnoli and Bergstrom [2005] Remark 2. In fact, the proof slightly alters from the proof of Lemma 1 of that paper. In addition, note that

$$
\lim _{\lambda^{+} \rightarrow \lambda_{L}} \frac{G_{\phi}(\lambda)}{G_{\phi}^{\prime}(\lambda)}=\frac{\lambda_{L}}{\lim _{\lambda^{+} \rightarrow \lambda_{L}} F_{\phi}\left(\lambda_{L}\right)} \geq \lambda_{L}
$$

so the solution must exist. Hence, if the solution is above $1, \lambda_{*}=1, x_{*}=q_{t} E_{\phi}[\lambda(\tilde{z})]$, and otherwise, it is given by the solution to (46) and (10). Finally, the fact that the solution is monotone in $\phi$ is a direct consequence of $\frac{G_{\phi}(\lambda)}{G_{\phi}^{\prime}(\lambda)}$ being monotone in $\phi$, as required by condition (iii) in 2 .

\section{E Proof of Proposition 4}

We show the result for $w_{t}^{i}$ by substituting results from previous propositions into (42). First observe that $x_{t}=x_{c} F_{\phi}\left(\lambda_{c}\right) n_{t}(z)$ since by Proposition 20, the agent sells $F_{\phi}\left(\lambda_{c}\right) n_{t}(z)$ in equity and obtains $x_{c}$ of liquid assets. Therefore, (42) is written as

$$
w_{2, t}=r_{t} n_{t}(z)+x_{c} F_{\phi}\left(\lambda_{c}\right) n_{t}(z)+q_{t}^{R}\left(\lambda(z) k_{t}(z)+\int_{0}^{1}\left(e_{2, t}^{+}(\tilde{z}, z)-e_{2, t}^{-}(\tilde{z}, z)\right) d \tilde{z}\right)
$$

from Proposition 16, the $\lambda(z) k_{t}(z)-\int_{0}^{1} e_{2, t}^{-}(\tilde{z}, z) d \tilde{z}=0$, so we have that the equation above becomes,

$$
=r_{t} n_{t}(z)+x_{c} n_{t}(z)+q_{t}^{R} \int_{0}^{1} e_{2, t}^{+}(\tilde{z}, z) d \tilde{z}
$$


Using Proposition 3 we have that

$$
\int_{0}^{1} e_{2, t}^{+}(\tilde{z}, z) d \tilde{z}=\int_{0}^{1} I\left[\lambda(\tilde{z}) \geq \lambda_{c}\right] e_{1, t}^{+}(\tilde{z}, z) d \tilde{z}
$$

and using 16 once again we have this quantity is equal to,

$$
\begin{aligned}
q_{t}^{R} n(z) \int_{0}^{1} I\left[\lambda(\tilde{z}) \geq \lambda_{c}\right] \lambda(\tilde{z}) g(\tilde{z}) d \tilde{z} & =q_{t}^{R} n(z)\left(1-F_{\phi}\left(\lambda_{c}\right)\right) E_{\phi}\left[\lambda(\tilde{z}) \mid \lambda(\tilde{z}) \geq \lambda_{c}\right] \int_{0}^{1} g(\tilde{z}) d \tilde{z} \\
& =q_{t}^{R} n(z)\left(1-F_{\phi}\left(\lambda_{c}\right)\right) E_{\phi}\left[\lambda(\tilde{z}) \mid \lambda(\tilde{z}) \geq \lambda_{c}\right]
\end{aligned}
$$

where the first part uses the Lemma 1 and the second follows from the fact that $\int_{0}^{1} g(\tilde{z}) d \tilde{z}$ is a density measure. Finally, using 20 we obtain that,

$$
w_{t}^{i}=\left(r_{t}+q_{t} E_{\phi}\left[\lambda(\tilde{z}) \mid \lambda(\tilde{z}) \leq \lambda_{c}\right] F_{\phi}\left(\lambda_{c}\right)+q_{t}^{R}\left(1-F_{\phi}\left(\lambda_{c}\right)\right) E_{\phi}\left[\lambda(\tilde{z}) \mid \lambda(\tilde{z}) \geq \lambda_{c}\right]\right) n(z)
$$

The definition of $w_{t}^{s}$ follows directly from the fact that s-entrepreneurs don't participate in the first stage and the definition of their budget constraint. Finally, the fact that both budgets are identical when $q_{t}=1$ follows from the fact that $\lambda_{c}=\lambda_{L}$ when this is the case.

\section{F Proof of Lemma 2}

Suppose $q_{t}<1$ for some $t$. Every entrepreneur would find the following optimal: since $q_{t}<1$, an entrepreneur can purchase outside equity $\Delta\left(e_{t+1}^{+}\right)$by selling the capital stock of it's firm, $q_{t} k_{t}$. This gives the entrepreneur an increase of $\left(1-q_{t}\right)$ in its equity. Since every entrepreneur finds this policy optimal, it is clearly not possible in equilibrium. Therefore, $q_{t} \geq 1$. Assume, $q_{t}>\frac{1}{\theta}$. Then, then the constraint 40 of the financing problem never binds. Thus, the a solution $i_{t}^{s}$ to the financing problem does not exist.

Assume that $q_{t} \in\left(1, \frac{1}{\theta}\right)$ and that the liquidity constraint does not bind. Then, the agent can sell an amount $x_{t}$, of equity. If it increases it's down payment by $\rho x_{t}$, it will increase it's equity tomorrow by

$$
\left[\frac{(1-\theta)}{\left(1-\theta q_{t}\right)} \rho-1\right] x_{t}
$$

Setting $\rho=\frac{\left(1-\theta q_{t}\right)}{(1-\theta)}$, it's future equity holdings are constant. But since $q_{t}>1, \rho<1$, so it can increase it's consumption in a strictly positive amount $(1-\rho) x_{t}$, and have the same continuation utility $E\left[V\left(w_{t+1}\right)\right]$.

Finally assume, $q_{t}=1$. Observe that (??) becomes ?? for the saving entrepreneur. Both the saving and the investing entrepreneur's problem must be identical unless the i-entrepreneurs constraint bind. If constraints, don't bind, both entrepreneurs have the same policy functions and investment must be cleared by the equity market.

\section{G Proof of Corollary 1}

From 2, note that when $q=1$, budget constraints for both agents problems are identical. There are no potential gains from trade. When $q>1$, the liquidity constraint binds. It suffices to show that there exists an incentive compatible and feasible change in the allocations, such that at 
least a single entrepreneur is better off. The candidate allocation requires are $\tilde{c}_{s}=c_{s}-(\theta+y) \varepsilon$, $\tilde{c}_{i}=c_{i}-(1-\theta+x-y) \varepsilon$ and $\tilde{\imath}_{s}=i_{s}+(\theta) \varepsilon \tilde{\imath}_{i}=i_{i}+(1-\theta+x) \varepsilon$

The candidate allocation is feasible since $\tilde{c}_{s}+\tilde{c}_{i}+\tilde{\imath}_{s}+\tilde{\imath}_{i}=c_{s}+c_{i}+i_{s}+i_{i}$. It is also incentive compatible since $\frac{\tilde{i}_{i}}{\tilde{\imath}_{s}}>\frac{i_{i}+(1-\theta) \varepsilon}{i_{s}+(\theta) \varepsilon}>\frac{(1-\theta)}{(\theta)} \frac{i+\varepsilon}{i+\varepsilon}=\frac{(1-\theta)}{(\theta)}$. It remains to be show that both agents are better-off. The euler equations in problems 5 and 6 are of the form: $q_{t} U^{\prime}\left(c_{t}^{s}\right)=V_{t}$ and $q_{t}^{R} U^{\prime}\left(c_{t}^{i}\right)=V_{t}$ for the saving entrepreneur and i-entrepreneurs respectively.

One can find a pair $(y, x), x>y>0$, satisfying the following two conditions

$$
\frac{(1-\theta+x-y)}{(1-\theta+x)}<q_{t}^{R} \text { and } \frac{(\theta+y)}{\theta}<q_{t}
$$

. Using the definition of $q_{t}^{R}$, these conditions are equivalent to finding a pair $(y, x)>0$ satisfying,

$$
\frac{1-\theta+y}{1-\theta}>q_{t}^{R}>\frac{(1-\theta+x-y)}{(1-\theta+x)}
$$

so one can find such pair.

Since $q_{t}^{R} \in(0,1)$, it is possible to find such pair. Finally, these conditions imply that:

$$
\begin{aligned}
V_{t}(\theta)-U^{\prime}\left(c_{t}^{s}\right)(\theta+y) & >0 \\
V_{t}(1-\theta+x)-U^{\prime}\left(c_{t}^{i}\right)(1-\theta+x-y) & >0
\end{aligned}
$$

By continuity of $U$ and $V$ this conditions imply that $\exists \varepsilon$ sufficiently small, such that the candidate allocation is constrained Pareto improving. The fact that the perturbation increases investment implies that there is under-investment.

\section{H Proof of Optimal Policies (Propositions 6,5 and 7 and Corol- laries 3 and ??)}

This section provides a proof of the optimal policies described in section 3 . The strategy is to guess and verify. For saving entrepreneurs, the guess is: $V(w ; t)=U\left(a_{t}^{s} w\right), c^{s}(w ; t)=\left(1-\varsigma_{t}^{s}\right) w, n_{t+1}^{s}=$ $\frac{\varsigma_{t}^{s} w}{q_{t}}$ and for the investing entrepreneur, the guess is : $V^{i}\left(w_{t}^{i}\right)=U\left(a_{t}^{i} w_{t}^{i}\right), c^{i}\left(w_{t}^{i} ; t\right)=\left(1-\varsigma_{t}^{i}\right) w_{t}^{i}$, $n_{t+1}^{i}\left(w_{t}^{i}\right)=\frac{\varsigma_{t}^{i} w_{t}^{i}}{q_{t}^{R}}$ and where $i_{t}=\gamma\left(n_{t+1}, n_{t}\right)$ is given by the liquidity constraint in binding states.

\section{H.1 Step 1: Optimal Portfolio Problem for Saving Type}

Using this guess, the first order conditions for $n_{t+1}^{s}$ is:

$$
\begin{aligned}
\left(n^{s \prime}\right): \quad & U^{\prime}(c)=\beta\left(E_{t}\left[\pi \Upsilon\left(a_{t}^{s} w_{t+1}^{s}\right)+(1-\pi) \Upsilon\left(a_{t}^{i} w_{t+1}^{i}\right)\right]\right)^{\frac{\gamma-1 / \theta}{1-\gamma}} \ldots \\
& \left((1-\pi) \Upsilon^{\prime}\left(a_{t+1}^{s} w_{t+1}^{s}\right) a_{t+1}^{s} \frac{r_{t+1}+q_{t+1} \lambda}{q_{t}}+\pi \Upsilon^{\prime}\left(a_{t+1}^{i} w_{t+1}^{i}\right) a_{t+1}^{i} \frac{r_{t+1}+q_{t+1}^{i} \lambda}{q_{t}}\right)
\end{aligned}
$$

Here, the fact that the investment opportunities are independent of other random variables is used. There are four types of returns that map current wealth in both states to wealth in both states. These maps are given by $w_{t+1}^{s}=R_{t+1}^{s s} \varsigma_{t}^{s} w_{t}^{s}, w_{t+1}^{s}=R_{t+1}^{i s} \varsigma_{t}^{i} w_{t}^{i}, w_{t+1}^{i}=R_{t+1}^{i i} \varsigma_{t}^{i} w_{t}^{i}$, and $w_{t+1}^{s}=R_{t+1}^{s i} \varsigma_{t}^{i} w_{t}^{s}$ where 


$$
R_{t}^{s s} \equiv \frac{\left(r_{t}+q_{t+1} \lambda\right)}{q_{t}}, R_{t}^{i s} \equiv \frac{\left(r_{t}+q_{t+1} \lambda\right)}{q_{t}^{R}}, R_{t}^{i i} \equiv \frac{\left(r_{t}+q_{t+1}^{i} \lambda\right)}{q_{t}^{R}} \text { and } R_{t}^{i s} \equiv \frac{\left(r_{t}+q_{t+1}^{i} \lambda\right)}{q_{t}}
$$

With these definitions, $w_{t}^{s}$ may is factored out from (47) to obtain:

$$
\begin{aligned}
\left(n^{\prime, s}\right): & U^{\prime}(c)=\beta\left(\varsigma_{t}^{s} w_{t}\right)^{-1 / \theta}\left(E_{t}\left[(1-\pi) \Upsilon\left(a_{t+1}^{s} R_{t+1}^{s s}\right)+\pi \Upsilon\left(a_{t+1}^{i} R_{t+1}^{s i}\right)\right]\right)^{\frac{\gamma-1 / \theta}{1-\gamma}} \ldots \\
& E_{t}\left[(1-\pi) \Upsilon^{\prime}\left(a_{t+1}^{s} R_{t+1}^{s s}\right) a_{t+1}^{s} R_{t+1}^{s s}+\pi \Upsilon^{\prime}\left(a_{t+1}^{i} R_{t}^{s i}\right) a_{t+1}^{i} R_{t}^{s i}\right]
\end{aligned}
$$

By replacing the guess for consumption and clearing out wealth, an Euler equation in terms of the marginal propensities to save is obtained:

$$
\begin{aligned}
\left(1-\varsigma_{t}^{s}\right)^{-1 / \theta}= & \beta\left(\varsigma_{t}^{s}\right)^{1-1 / \theta}\left(E_{t}\left[(1-\pi) \Upsilon\left(a_{t+1}^{s} R_{t}^{s s}\right)+\pi \Upsilon\left(a_{t+1}^{i} R_{t}^{s i}\right)\right]\right)^{\frac{\gamma-1 / \theta}{1-\gamma}} \ldots \\
& E_{t}\left[(1-\pi) \Upsilon^{\prime}\left(a_{t+1}^{s} R_{t}^{s}\right) a_{t+1}^{s} R_{t}^{s s}+\pi \Upsilon^{\prime}\left(a_{t+1}^{i} R_{t}^{i}\right) a_{t+1}^{i} R_{t}^{s i}\right]
\end{aligned}
$$

\section{H.2 Step 2: Optimal Portfolio Problem for Investing Type}

Lemma 2 shows that when $q>1$, liquidity constraints for i-entrepreneurs binds. This enables to express the i-entrepreneurs budget constraint solely in terms of future equity by equation (??). Proceeding in the same way as in step 1, the first order condition for future equity in the investment state as a function of the marginal propensities to consume is given by:

$$
\begin{aligned}
\left(1-\varsigma_{t}^{i}\right)^{-1 / \theta}= & \frac{\beta}{q_{t}^{R}}\left(\varsigma_{t}^{i}\right)^{1-1 / \theta}\left(E_{t}\left[(1-\pi) \Upsilon\left(a_{t+1}^{s} R_{t}^{i s}\right)+\pi \Upsilon\left(a_{t+1}^{i} R_{t}^{i i}\right)\right]\right)^{\frac{\gamma-1 / \theta}{1-\gamma}} \\
& E_{t}\left[(1-\pi) \Upsilon^{\prime}\left(a_{t+1}^{s} R_{t}^{s}\right) a_{t+1}^{s} R_{t}^{i s}+\pi \Upsilon^{\prime}\left(R_{t}^{i}\right) a_{t+1}^{i} R_{t}^{i i}\right]
\end{aligned}
$$

\section{H.3 Step 3: Verification of the Value function}

Replacing the guesses and by using the envelope theorem the following relation in the saving state is obtained:

$$
U^{\prime}\left(\left(1-\varsigma_{t}^{s}\right) w_{t}\right)=V_{w}^{\prime}\left(a_{t}^{s} w_{t}\right) a_{t}^{s} \rightarrow\left(a_{t}^{s}\right)^{1-\frac{1}{\theta}}=\left(1-\varsigma_{t}^{s}\right)^{-\frac{1}{\theta}}
$$

Similarly, for the investment state:

$$
\left(a_{t}^{i}\right)^{1-\frac{1}{\theta}}=\left(1-\varsigma_{t}^{i}\right)^{-\frac{1}{\theta}}
$$

Rewriting both relations gives us or $a_{t}^{j}=\left(1-\varsigma_{t}^{j}\right)^{\frac{1}{1-\theta}}, j=i, s$. The certainty equivalent of a unit of wealth tomorrow in the saving state is given by the function:

$$
\Omega^{s}\left(a_{t+1}^{s}, a_{t+1}^{i}\right)=\Upsilon^{-1} E_{t}\left[(1-\pi) \Upsilon\left(a_{t+1}^{s} R_{t+1}^{s s}\right)+\pi \Upsilon\left(a_{t+1}^{i} R_{t+1}^{s i}\right)\right]
$$

which is homogeneous of degree 1 . The same is true about:

$$
\Omega^{i}\left(a_{t+1}^{s}, a_{t+1}^{i}\right)=\Upsilon^{-1} E_{t}\left[(1-\pi) \Upsilon\left(a_{t+1}^{s} R_{t+1}^{i s}\right)+\pi \Upsilon\left(a_{t+1}^{i} R_{t+1}^{i i}\right)\right]
$$


The recursions (49) and (50) given by the euler equations of the entrepreneurs may be written in terms of this random variables to deliver:

$$
\left(1-\varsigma_{t}^{s}\right)^{-\frac{1}{\theta}}=\beta\left(\varsigma_{t}^{s}\right)^{-1 / \theta} \Omega^{s}\left(a_{t+1}^{s}, a_{t+1}^{i}\right)^{1-1 / \theta}
$$

and

$$
\left(1-\varsigma_{t}^{i}\right)^{-\frac{1}{\theta}}=\beta\left(\varsigma_{t}^{s}\right)^{-1 / \theta} \Omega^{i}\left(a_{t+1}^{s}, a_{t+1}^{i}\right)^{1-1 / \theta}
$$

Clearing out $\varsigma_{t}^{s}$ from the right hand side, and adding 1 to both sides yields:

$$
\left(1-\varsigma_{t}^{s}\right)^{-1}=1+\beta \Omega^{s}\left(a_{t+1}^{s}, a_{t+1}^{i}\right)^{\theta-1}
$$

and

$$
\left(1-\varsigma_{t}^{i}\right)^{-1}=1+\beta \Omega^{i}\left(a_{t+1}^{s}, a_{t+1}^{i}\right)^{\theta-1}
$$

where $\Omega_{t}$ is a shorthand for $\Omega\left(a_{t+1}^{s}, a_{t+1}^{i}\right)$. Note that any value function satisfies the envelope condition so any pair of functions $\varsigma_{t}^{s}, \varsigma_{t}^{i}$ satisfying this recursion guarantee that the functional form guessed for $V^{i}$ and $V^{s}$ satisfy the Bellman equation.

Since policies are independent of wealth we have shown that,

Corollary 3 (Aggregation) The economy admits a aggregation.

\section{H.4 Step 4: Uniqueness of policy functions}

Fix the price functions $q^{i}(s), q^{R}(s), q(s)$. To simplify notation and let $x(s)=\left(1-\varsigma^{i}(s)\right)^{-1}$ and $y(s)=\left(1-\varsigma^{s}\right)^{-1}$ so that recursions (49) and (50) become:

$$
\begin{aligned}
& x(s)=1+(\beta)^{\theta} \Omega_{t}^{s}\left(x(s)^{\frac{1}{\theta-1}}, y(s)^{\frac{1}{\theta-1}}\right)^{\theta-1} \\
& y(s)=1+(\beta)^{\theta} \Omega_{t}^{i}\left(x(s)^{\frac{1}{\theta-1}}, y(s)^{\frac{1}{\theta-1}}\right)^{\theta-1}
\end{aligned}
$$

where $\Omega_{t}^{s}$ and $\Omega_{t}^{i}$ are defined in the previous step. These equations define an operator $T: \mathbb{S}^{2} \rightarrow$ $\mathbb{S}^{[1, \infty]^{2}}$, that maps continuous bounded functions of the state $S$ to $\mathbb{R}_{+}^{2}$ into two continuous functions of the state $S$ taking values in $[1, \infty]$. Since $\mathbb{S}$ is compact, then $\mathbb{S}^{2}$ is a complete metric space with respect to the sup-norm for the product space. Thus, $T$ defines a recursion:

$$
\left(x_{t+1}, y_{t+1}\right)=T\left(x_{t}, y_{t}\right)
$$

for any $x_{o}$ and $y_{o}$ initial functions in $\mathbb{S}^{\mathbb{R}_{+}^{2}}$. We check that the recursion satisfies Blackwell's sufficient conditions.

Monotonicity. When $\theta<1$, the power function $z^{\frac{1}{\theta-1}}$ is decreasing. Thus, for any $x>x$ we have: $x^{\prime}(s)^{\frac{1}{\theta-1}}<x(s)^{\frac{1}{\theta-1}}$ so the certainty equivalent with respect to $\Upsilon$ for $x \prime(s)$ is smaller. Hence, $\Omega_{t}^{s}\left(x(s)^{\frac{1}{\theta-1}}, y(s)^{\frac{1}{\theta-1}}\right)<\Omega_{t}^{s}\left(x^{\prime}(s)^{\frac{1}{\theta-1}}, y(s)^{\frac{1}{\theta-1}}\right)$. Since $\theta-1$ is negative, then $\Omega_{t}^{s}\left(x(s)^{\frac{1}{\theta-1}}, y(s)^{\frac{1}{\theta-1}}\right)^{\theta-1}>$ $\Omega_{t}^{s}\left(x^{\prime}(s)^{\frac{1}{\theta-1}}, y(s)^{\frac{1}{\theta-1}}\right)^{\theta-1}$. If $\theta>1$, the argument is direct. The same is true for $\Omega_{t}^{i}$.

Discounting. Assume that $\frac{\gamma-\theta}{\theta-1}>0$. Then, observe that $\Omega_{t}^{i}\left(x(s)^{\frac{1}{\theta-1}}, y(s)^{\frac{1}{\theta-1}}\right)^{\theta-1}$ is the certainty equivalent of a CRRA function with exponent $\frac{\gamma-\theta}{\theta-1}$. Under the assumption $\frac{\gamma-\theta}{\theta-1}>0$, this 
function has increasing absolute risk aversion. Proposition 6.C.3 in Mas-Collel, Winston and Green asserts that :

$$
\Omega_{t}^{s}\left([x(s)+a]^{\frac{1}{\theta-1}},[y(s)+a]^{\frac{1}{\theta-1}}\right)^{\theta-1}<a+\Omega_{t}^{s}\left([x(s)]^{\frac{1}{\theta-1}},[y(s)]^{\frac{1}{\theta-1}}\right)^{\theta-1}
$$

so $T(x+a, y+a)<\beta^{\theta} a+T(x+a, y+a)$. Finally, for the case in which $\frac{\gamma-\theta}{\theta-1}<0$, one has to be more careful. Discounting will not be met directly, but for a sufficiently small neighborhood. A formal argument would extend this to the whole domain.

Alternative proof. Additional notation is required. Call $\bar{x}_{t}, \bar{y}_{t}$ the recursion that begins with $x_{o}(s)=\infty$ and $y_{o}(s)=\infty$ all $s$ and let $\tilde{x}_{t}, \tilde{y}_{t}$ the recursion that begging with $x_{o}(s)=1$ and $y_{o}(s)=1$ any $s$. Finally, is used $\succ$ to denote the partial ordering of $\boldsymbol{R}^{2}$. There are two cases of interest: $\theta>1$ and $\theta<1$. The case where $\theta=1$ is immediate.

Case $\theta<1$. Note that $T\left(\bar{x}_{o}, \bar{y}_{o}\right)=\left(\tilde{x}_{o}, \tilde{y}_{o}\right)$ since $\Omega^{s}(\infty, \infty)^{\theta-1}=0$. In addition, note that $\Omega^{i}$ and $\Omega^{s}$ are increasing functions of both $x$ and $y$. Hence, if for some pair $(x, y) \succ(v, c)$ one has that $T\left(x_{1}, y_{1}\right) \prec T(v, c)$ since $\left(\Omega^{s}\right)^{\theta-1}$ is decreasing in $\Omega^{s}$, and $\Omega^{s}$ is increasing in both $x$ and $y$. Again, by the same token, $T^{2}(x, y) \succ T^{2}(v, c)$. Thus, by induction:

$$
\left(\bar{x}_{t}, \bar{y}_{t}\right) \succ\left(x_{t}, y_{t}\right) \succ\left(\tilde{x}_{t}, \tilde{y}_{t}\right) \text { for } t \text { even }
$$

and

$$
\left(\bar{x}_{t}, \bar{y}_{t}\right) \prec\left(x_{t}, y_{t}\right) \prec\left(\tilde{x}_{t}, \tilde{y}_{t}\right) \text { for } t \text { odd }
$$

Note that $\left(\tilde{x}_{1}, \tilde{y}_{1}\right) \prec(\infty, \infty)$ since $\Omega^{s}(1,1)$ is bounded and strictly positive since prices are bounded. Since $\left(\tilde{x}_{1}, \tilde{y}_{1}\right) \prec\left(\bar{x}_{0}, \bar{y}_{0}\right)$ then $\left(\tilde{x}_{2}, \tilde{y}_{2}\right) \succ\left(\tilde{x}_{0}, \tilde{y}_{0}\right)$. By induction, it follows that $\left(\tilde{x}_{2 t}, \tilde{y}_{2 t}\right)$ is an increasing sequence and that $\left(\tilde{x}_{2 t+1}, \tilde{y}_{2 t+1}\right)$ are increasing. Thus, even sequences are monotone increasing and the even sequences are monotonically increasing and both converge. Finally, one needs to show that as $t \rightarrow \infty,\left\|\left(\bar{x}_{t}, \bar{y}_{t}\right)-\left(\tilde{x}_{t}, \tilde{y}_{t}\right)\right\| \rightarrow 0$. Suppose that it is not the case. Since the sequences $\left(\tilde{x}_{2 t+1}, \tilde{y}_{2 t+1}\right),\left(\tilde{x}_{2 t}, \tilde{y}_{2 t}\right)$ are monotone, bounded and convergent. $\left\|\left(\bar{x}_{t}, \bar{y}_{t}\right)-\left(\tilde{x}_{t}, \tilde{y}_{t}\right)\right\|$ must converge. Suppose $\lim _{t \rightarrow \infty}\left\|\left(\bar{x}_{t}, \bar{y}_{t}\right)-\left(\tilde{x}_{t}, \tilde{y}_{t}\right)\right\| \neq 0$. Then, $\exists \Delta$ such that $\frac{\Delta}{2}<\left\|\left(\bar{x}_{t}, \bar{y}_{t}\right)-\left(\tilde{x}_{t}, \tilde{y}_{t}\right)\right\|, \forall t$. But since, $\left(\bar{x}_{t+1}, \bar{y}_{t+1}\right)=\left(\tilde{x}_{t}, \tilde{y}_{t}\right)$, this implies that

$$
\left\|\left(\tilde{x}_{t+1}, \tilde{y}_{t+1}\right)-\left(\tilde{x}_{t}, \tilde{y}_{t}\right)\right\|>\frac{\Delta}{2}
$$

and

$$
\left\|\left(\tilde{x}_{t+2}, \tilde{y}_{t+2}\right)-\left(\tilde{x}_{t+1}, \tilde{y}_{t+1}\right)\right\|>\frac{\Delta}{2}
$$

- By the triangle inequality, the assumption requires that $\left\|\left(\tilde{x}_{t+2}, \tilde{y}_{t+2}\right)-\left(\tilde{x}_{t}, \tilde{y}_{t}\right)\right\|>\Delta, \forall t$. This contradicts the fact that monotone bounded sequences converge. Thus,

$$
\lim _{t \rightarrow \infty}\left\|\left(\bar{x}_{t}, \bar{y}_{t}\right)-\left(\tilde{x}_{t}, \tilde{y}_{t}\right)\right\| \neq 0 .
$$

since convergence is uniform, for all $s$,

Case $\theta>1: \Omega$ is decreasing in $x$ and $y$ and therefore $T$ is increasing. Define again $\left\{\tilde{x}_{t}, \tilde{y}_{t}\right\}$ as the sequence defined by $T$ starting from $(1,1)$. If $\left\{\tilde{x}_{t}, \tilde{y}_{t}\right\}$ is unbounded. The solution is $\tilde{x}_{t}, \tilde{y}_{t}=(\infty, \infty)$. If it is bounded, then it has a fixed point.as monotone bounded functions converge. Suppose there are to such fix points. Then, $T(\tilde{x}, \tilde{y})<T(\bar{x}, \bar{y})$ by construction. Then, since $T(\tilde{x}, \tilde{y})$ are positive, one can find a constant $\aleph>1$ such that $(\aleph \tilde{x}, \aleph \tilde{y})>(\bar{x}, \bar{y})$ and therefore, $T(\aleph \tilde{x}, \aleph \tilde{y})>T(\tilde{x}, \tilde{y})$. By homogeneity of $\Omega$ this requires that $\aleph^{-1} \Omega(\tilde{y}, \tilde{x})^{\theta-1}>\Omega(\bar{x}, \bar{y})^{\theta-1}$ or $(\tilde{y}, \tilde{x})>(\bar{y}, \bar{x})$ which contradicts the fact that $T$ is monotone. 
Since any sequence $\left(x_{t}, y_{t}\right)$ is lies within $\left(\bar{x}_{t}, \bar{y}_{t}\right) \times\left(\tilde{x}_{t}, \tilde{y}_{t}\right)$ one has that:

$$
\lim _{t \rightarrow \infty}\left(\tilde{x}_{t}, \tilde{y}_{t}\right)=\left(x^{*}, y^{*}\right)
$$

where $\left(x^{*}, y^{*}\right)$ are a the unique fixed point of the operator $T$. This provides an algorithm to compute $\tilde{x}_{t}, \tilde{y}_{t}$ and ensures that policy functions $\varphi^{i}(s), \varphi^{s}(s)$ are unique.

\section{Returns and State Equations}

Labor Demand: Taking their capital stock $k$ as given firms are run efficiently. One obtains aggregate labor demand by taking first order conditions and aggregating over the set of entrepreneurs.

$$
L^{d}(s)=\left[\frac{A(1-\alpha)}{\omega(s)}\right]^{\frac{1}{\alpha}} K
$$

Equilibrium Employment: Workers consumption is $c=\omega(s) L^{s}(s)$ where $L^{s}(s)$ is the labor supply. The solution to the workers problem defines their labor supply. Equilibrium employment pins down the wage, $\omega(s)=\bar{\omega}^{\frac{\alpha}{\alpha+\nu}}[A(1-\alpha)]^{\frac{\nu}{\alpha+\nu}} K^{\frac{\nu \alpha}{\alpha+\nu}}$ and equilibrium employment $L^{*}(A, K)=$ $\left[\frac{(1-\alpha)}{\bar{\omega}}\right]^{\frac{1}{\alpha+\nu}}\left[A^{\frac{1}{\alpha}} K\right]^{\frac{\alpha}{\alpha+\nu}}$.

Returns to Equity: Profits per firm are obtained substituting the equilibrium labor demand and wages into the profit function:

$$
r(s)=[A]^{\frac{\xi+1}{\alpha}} \bar{\omega}^{\frac{\xi}{\nu}} K^{\xi} \Gamma(\alpha)
$$

where $\xi=\frac{\nu(\alpha-1)}{\alpha+\nu}<0$ ensures decreasing returns to scale at the aggregate level. The term $\Gamma(\alpha)=$ $[(1-\alpha)]^{\frac{(\nu+1)}{(\alpha+\nu)}}\left[\frac{1}{(1-\alpha)}-1\right]$ is a function of the technology and the Frisch-elasticity. ${ }^{21}$ Aggregate dynamics are obtained by aggregating the policy functions described in the previous section. The equilibrium is characterized without the need of keeping track of distributions because the policies are linear functions wealth.

Aggregate Output: Total output is the sum of the return to labor and the return to capital. Return to workers is $w(s) L^{s}(s)=\bar{\omega}^{\frac{\alpha-1}{\alpha+\nu}}[A(1-\alpha)]^{\frac{\nu+1}{\alpha+\nu}} K^{\xi+1}$. Equating (51) to $L^{s}(s)$ in this expression and using (52), we obtain the return to equity $r(s)$ implicitly by solving $r(s) K=\bar{r}[A]^{\frac{\xi+1}{\alpha}} K^{\xi+1}$. Aggregate output is the sum of the capital share and labor shares,

$$
Y(s)=\left(A^{a}+\bar{\omega}^{\frac{\alpha-1}{\alpha+\nu}}[A(1-\alpha)]^{\frac{\nu+1}{\alpha+\nu}}\right) K^{\gamma+1}
$$

Since, $0<\xi+1<1$, this ensures that aggregate output features decreasing returns to scale, which ensures that $\mathbb{K}$ is bounded.

Aggregate Variables: Corollary 3 derived in the appendix shows that $K^{\prime}=N^{i}+N^{s}$, where $\left(N^{i}, N^{s}\right)$ are the equity holdings of i and s entrepreneurs. Using the results from Propositions 5 and 6 consumption and aggregate equity evolve according to:

$$
\begin{aligned}
C^{s}(s) & =\left(1-\varsigma^{s}(s)\right)\left(r(s)+q(s) E_{\phi}[\lambda]\right)(1-\pi) K \\
q(s) N^{\prime, s}(s) & =\varsigma^{s}(s)\left(r(s)+q(s) E_{\phi}[\lambda]\right)(1-\pi) K
\end{aligned}
$$

\footnotetext{
${ }^{21}$ The closer $\alpha$ is to 1 , the more elastic are profits to aggregate capital and lower the returns on average. Notice also that the larger the marginal rate of substitution $\bar{\omega}$, the lower are average returns. This parameters allows us to control the relative income of entrepreneurs to workers.
} 
and

$$
\begin{aligned}
C^{i}(s) & =\left(1-\varsigma^{i}(s)\right)\left(r(s)+E_{\phi}[\lambda] q^{i}(s)\right) \pi K \\
q^{R}(s) N^{i}(s) & =\varsigma^{i}(s)\left(r(s)+E_{\phi}[\lambda] q^{i}(s)\right) \pi K
\end{aligned}
$$

The next section describes the characteristics of this equilibrium.

\section{J Proof of Proposition 8}

We use equation (quadratic) clearing out $I_{t}^{s}\left(s_{t}\right)$ and $I_{t}^{d}\left(s_{t}\right)$ and substituting in the equilibrium conditions found in 5 and 6 we have that $q^{*}$ must solve.

$$
\frac{(1-\pi)\left(\varsigma_{t}^{s}\left(r_{t}+q_{t} E_{\phi}[\lambda]\right) K_{t} / q_{t}-E_{\phi}[\lambda] K_{t}\right)-\pi \varphi_{t} K_{t}}{\theta}=\frac{\pi\left[\varsigma_{t}^{i}\left(r_{t}+q_{t}^{i} E_{\phi}[\lambda] K_{t}\right) / q_{t}^{R}-\psi_{t} K_{t}\right]}{(1-\theta)}
$$

Clearing out $K_{t}$ from both sides, using (11) this expression becomes,

$$
\begin{aligned}
\frac{(1-\pi) \varsigma_{t}^{s} r_{t}-\left((1-\pi)\left(1-\varsigma_{t}^{s}\right) E_{\phi}[\lambda]+\pi \varphi_{t}\right) q_{t}}{\theta q_{t}} & =\frac{\pi\left[\varsigma_{t}^{i}\left(r_{t}+q_{t} \varphi_{t}\right)-\left(1-\varsigma_{t}^{i}\right) \psi_{t} q_{t}^{R}\right]}{(1-\theta) q_{t}^{R}} \\
& =\frac{\pi \varsigma_{t}^{i}\left(r_{t}+q_{t} \varphi_{t}\right)}{\left(1-\theta q_{t}\right)}-\frac{\pi\left(1-\varsigma_{t}^{i}\right) \psi_{t}}{(1-\theta)}
\end{aligned}
$$

and by getting rid of the denominators we rearrange terms to obtain,

$$
\begin{aligned}
& (1-\pi) \varsigma_{t}^{s} r_{t}\left(1-\theta q_{t}\right)-\left((1-\pi)\left(1-\varsigma_{t}^{s}\right) E_{\phi}[\lambda]+\pi \varphi_{t}\right) q_{t}\left(1-\theta q_{t}\right) \\
= & \theta q_{t} \pi \varsigma_{t}^{i}\left(r_{t}+q_{t} \varphi_{t}\right)-\theta q_{t}\left(1-\theta q_{t}\right) \pi \frac{\left(1-\varsigma_{t}^{i}\right) \psi_{t}}{(1-\theta)}
\end{aligned}
$$

From this equation we obtain a quadratic expression for $q_{t}$

$$
\begin{aligned}
& (1-\pi) \varsigma_{t}^{s} r_{t}-q_{t} \theta(1-\pi) \varsigma_{t}^{s} r_{t}-\left((1-\pi)\left(1-\varsigma_{t}^{s}\right) E_{\phi}[\lambda]+\pi \varphi_{t}\right) q_{t} \\
& +\theta q_{t}^{2}\left((1-\pi)\left(1-\varsigma_{t}^{s}\right) E_{\phi}[\lambda]+\pi \varphi_{t}\right) \\
= & q_{t} \theta \pi \varsigma_{t}^{i} r_{t}+q_{t}^{2} \theta \pi \varsigma_{t}^{i} \varphi_{t}-\theta q_{t} \frac{\left(1-\varsigma_{t}^{i}\right) \psi_{t}}{(1-\theta)}+q_{t}^{2} \theta^{2} \frac{\left(1-\varsigma_{t}^{i}\right) \psi_{t}}{(1-\theta)} \pi
\end{aligned}
$$

This expression defines a quadratic equation in $q^{*}$,

$$
\left(q^{*}\right)^{2} A+q^{*} B+C=0
$$

with coefficients,

$$
\begin{aligned}
A & =-\theta\left((1-\pi)\left(1-\varsigma_{t}^{s}\right) E_{\phi}[\lambda]+\pi\left(1-\varsigma_{t}^{i}\right) \varphi_{t}-\theta \frac{\left(1-\varsigma_{t}^{i}\right) \psi_{t}}{(1-\theta)} \pi\right) \\
B & =\theta r_{t}\left((1-\pi) \varsigma_{t}^{s}+\pi \varsigma_{t}^{i}\right)+\left((1-\pi)\left(1-\varsigma_{t}^{s}\right) E_{\phi}[\lambda]+\pi \varphi_{t}\right)-\theta \frac{\left(1-\varsigma_{t}^{i}\right) \psi_{t}}{(1-\theta)} \pi \\
C & =-(1-\pi) \varsigma_{t}^{s} r_{t}
\end{aligned}
$$


$C$ is clearly negative. A pointwise inspection of $A$ shows that this terms is also negative. By Assumption ?? $(1-\pi)>\frac{\theta \pi}{(1-\theta)}$ and by Proposition 7, we have that $\left(1-\varsigma_{t}^{i}\right)<\left(1-\varsigma_{t}^{s}\right)$. Finally, since $\varphi_{t}$ and $\varphi_{t}$ are positive, the term $\psi_{t} \theta-\varphi_{t}(1-\theta)<\psi_{t} \theta<\psi_{t}+\psi_{t}=E_{\phi}[\lambda]$. Finally, $B$ is also positive because,

$$
(1-\pi)\left(1-\varsigma_{t}^{s}\right) E_{\phi}[\lambda]-\theta \frac{\left(1-\varsigma_{t}^{i}\right) \psi_{t}}{(1-\theta)} \pi>(1-\pi)\left(1-\varsigma_{t}^{s}\right)\left(E_{\phi}[\lambda]-\psi_{t}\right) \geq 0
$$

Evaluated at $0,(54)$ is negative. It reaches a maximum at $-\frac{B}{2 A}>0$ and then will diverge to $-\infty$. The discriminant (54) is also positive. Observe that,

$$
B=B_{1}+B_{2}
$$

where

$$
B_{1}=\left((1-\pi)\left(1-\varsigma_{t}^{s}\right) E_{\phi}[\lambda]+\pi \varphi_{t}\right)-\theta \frac{\left(1-\varsigma_{t}^{i}\right) \psi_{t}}{(1-\theta)} \pi
$$

and

$$
B_{2}=\theta r_{t}\left((1-\pi) \varsigma_{t}^{s}+\pi \varsigma_{t}^{i}\right)
$$

So then, $B_{2}>\theta(1-\pi) \varsigma_{t}^{s} r_{t}$ and $B_{1}>\left((1-\pi)\left(1-\varsigma_{t}^{s}\right) E_{\phi}[\lambda]+\pi\left(1-\varsigma_{t}^{i}\right) \varphi_{t}-\theta \frac{\left(1-\varsigma_{t}^{i}\right) \psi_{t}}{(1-\theta)} \pi\right)$. Thus, $B^{2}-4 A C>B^{2}-4 B_{1} B_{2}=\left(B_{1}-B_{2}\right)^{2}>0$. Thus, the roots $\left(q_{1}, q_{2}\right)$ of $(54)$ are both positive. (18) is satisfied when (54) is negative. There are three cases.

Case 1: If $1>q_{1}$, then $q_{t}=1$ the aggregate incentive compatibility constraint is not binding at $q_{t}=1$.

Case 2: If $1 \in\left(q_{1}, q_{2}\right)$, then, $q_{t}=1$ violates the aggregate incentive compatibility constraint. Since for $q_{t}>1$, constraints hold with equality, is must be the case that $q_{t}=q_{2}$ is the unique value of $q_{t}$ satisfying the constraint (18) at equality.

Case 3: If $1<q_{1},(18)$ does not bind at $q_{t}=1$.

\section{K Proof of Proposition 9}

The proof is direct considering the monotonicity of $\varphi_{t}\left(s_{t}\right), \psi_{t}\left(s_{t}\right), \varsigma_{t}^{s}\left(s_{t}\right), \varsigma_{t}^{i}\left(s_{t}\right)$ as functions of $q_{t}$. In turn, $q_{t}$ in proposition 8 is monotonic in the right way.

Fix $s_{t}$ and the functions $q^{\prime}, \varphi^{\prime}, \psi^{\prime}, \varsigma^{s^{\prime}}, \varsigma^{i \prime}$. The left hand side of 7 defines functions $\varsigma_{t}^{s}(q), \varsigma_{t}^{i}(q)$. Similarly, the solution to (9) and (10) as functions of $q$, define a threshold depreciation $\lambda_{*}(q) \cdot \lambda_{*}$ defines in turn $\varphi, \psi$ via (12) and (13). Thus, implicitly,

$$
\begin{aligned}
\varphi(q) & \equiv \varphi\left(\lambda_{*}(q)\right) \\
\psi(q) & \equiv \psi\left(\lambda_{*}(q)\right)
\end{aligned}
$$

Finally, (20) implicitly defines a function of $q$ :

$$
q^{*}(q) \equiv q\left(\varphi\left(\lambda_{*}(q)\right), \psi\left(\lambda_{*}(q)\right), \varsigma_{t}^{s}(q), \varsigma_{t}^{i}(q)\right)
$$

One can verify immediately that $\lambda_{*}(q)$ is increasing in $q$. Therefore, $\varphi\left(\lambda_{*}(q)\right)$ is increasing and $\psi\left(\lambda_{*}(q)\right)$ is decreasing in $q$. On the other hand, $\varsigma_{t}^{s}(q)$ is decreasing and $\varsigma_{t}^{i}(q)$ is increasing in 
$q$. We are looking for the fixed point $q=q^{*}(q)$ in $q \in\left(0, \frac{1}{\theta}\right)$. It is enough to show that $q^{*}$ is monotonically decreasing in $q$, that $q^{*}(1) \geq 1$, and that $\lim _{q \rightarrow 1 / \theta} q^{*}(q)=1$. Since all of the functions $\varsigma_{t}^{s}(q), \varsigma_{t}^{i}(q), \varphi\left(\lambda_{*}(q)\right), \psi\left(\lambda_{*}(q)\right)$ are also monotonic, the fixed point is unique in the vector $\varphi_{t}\left(s_{t}\right), \psi_{t}\left(s_{t}\right), \varsigma_{t}^{s}\left(s_{t}\right), \varsigma_{t}^{i}\left(s_{t}\right), q\left(s_{t}\right)$.

Since $\varsigma_{t}^{s}(q)$ is decreasing and $\varphi\left(\lambda_{*}(q)\right)$ increasing in $q, I_{t}^{s}$ defined by (17) is decreasing in $q$. In turn, $\psi\left(\lambda_{*}(q)\right)$ is decreasing and $\varsigma_{t}^{i}(q)$ increasing in $q$ so $I_{t}^{d}$ defined by (??) is increasing in $q$. Therefore, as $q$, grows, $q^{*}(q)$ solving (20) must be smaller since (18) is satisfied with a smaller value for $q \cdot q^{*}(1) \geq 1$ by definition and finally, $\lim _{q \rightarrow 1 / \theta} I_{t}^{i}(q)=\infty$, so $\lim _{q \rightarrow 1 / \theta} q^{*}(q)=1$.

\section{Proof of Proposition 10 and Corollary 2}

Incomplete.

\section{Proof of Proposition 11}

If (18) never binds, then $q(s)=1$ constraints never bind. Then the recursions in Proposition 7 are identical and have a unique solution defined by $\varsigma^{*}$. Then, using (16) and (17) and (18) at equality yields the following condition:

$$
\theta \pi\left[\varsigma^{*}\left(r(A, K)+E_{\phi}[\lambda]\right)-\psi\right]-(1-\theta)(1-\pi)\left[\varsigma^{*}\left(r(A, K)+E_{\phi}[\lambda]\right)-E_{\phi}[\lambda]\right]+(1-\theta) \pi \varphi=0
$$

Using the fact that $\psi=E_{\phi}[\lambda]-\varphi$ we can collect $\varphi$ from both sides and obtain,

$$
\varphi=\frac{[(1-\pi)(1-\theta)-\theta \pi]}{\pi}\left(\varsigma^{*} r(A, K)-\left(1-\varsigma^{*}\right) E_{\phi}[\lambda]\right)
$$

and by definition, $\varphi$ is given by, $E_{\phi}\left[\lambda(\tilde{z}) \mid \lambda(\tilde{z})<\lambda_{c}\right] F\left(\lambda_{c}\right)$. By monotonicity of this last term, $\lambda_{c}$ is the unique solution to,

$$
E_{\phi}\left[\lambda(\tilde{z}) \mid \lambda(\tilde{z})<\lambda_{c}\right] F\left(\lambda_{c}\right) \equiv \frac{[(1-\pi)(1-\theta)-\theta \pi]}{\pi}\left(\varsigma^{*} r(A, K)-\left(1-\varsigma^{*}\right) E_{\phi}[\lambda]\right)
$$

To finance contracts implementing such level of liquidity, financial firms need funds equivalent to $\left(\lambda_{c}-E_{\phi}\left[\lambda(\tilde{z}) \mid \lambda(\tilde{z})<\lambda_{c}\right]\right) \geq 0$, since, $\lambda_{c}$, is the opportunity cost of selling equity of the threshold firm and $E_{\phi}\left[\lambda(\tilde{z}) \mid \lambda(\tilde{z})<\lambda_{c}\right]$ the return to financial firms. Thus, the zero profit condition requires such an amount of external financing.

\section{N Proof of Proposition 14 (log preference case)}

The proof is similar to the proof of 8 Notice that the supply and demand equations can be normalized by deviding both sides by $\mathrm{K}$ :

$$
i^{s}(q)=(1-\pi)\left(\beta \frac{r}{q}-(1-\beta) E_{\phi}[\lambda(\phi)]\right)-\pi\left(\frac{\left(\lambda B-B^{\prime}\right)}{K}+\varphi\left(x_{c}, s\right)\right)
$$

and

$$
i^{i}(q)=\pi\left[\beta \frac{r+q \varphi\left(x_{c}, s\right)}{q^{R}}-(1-\beta) \psi\left(x_{c}, s\right)\right]
$$


where $i^{s}(q)$ and $i^{i}(q)$ are the investments of savers and investors per unit of capital. $\frac{\left(\lambda B-B^{\prime}\right)}{K}$ can be expressed in terms of new state variable:

$$
\frac{\left(\lambda B-B^{\prime}\right)}{K}=\lambda b-b^{\prime} \frac{K^{\prime}}{K}=\lambda\left(b-b^{\prime}\right)-b^{\prime}\left(i^{i}(q)+i^{s}(q)\right)
$$

where $b=\frac{B}{K}$. Thus, this condition determines the per-capita behavior of both quantities.

The incentive compatibility constraint now reads:

$$
\begin{aligned}
& (1-\pi)\left(\beta \frac{r}{q}-(1-\beta) E[\lambda]\right)-\pi\left(\lambda\left(b-b^{\prime}\right)+\varphi\right) \\
\leq & {\left[\frac{(1-\theta)+(2-\theta) b^{\prime}}{\theta}\right] \pi\left[\beta \frac{r+q \varphi}{q^{R}}-(1-\beta) \psi\right] }
\end{aligned}
$$

Define $H=\left[\frac{(1-\theta)+(2-\theta) b^{\prime}}{\theta}\right]$. Then, using the definition of $q^{R}, q$ will solve a quadratic equation.

$$
\begin{aligned}
& (1-\theta q)(1-\pi)(\beta r-q(1-\beta) E[\lambda])-q \pi\left(\lambda\left(b-b^{\prime}\right)+\varphi\right) \\
\leq & q H \pi\left[\beta(r+q \varphi)(1-\theta)-\frac{(1-\beta) \psi}{(1-\theta)}(1-\theta q)\right]
\end{aligned}
$$

where the quadratic terms are:

$$
\begin{gathered}
A=-\theta\left[(1-\beta)(1-\pi) E[\lambda]+\pi\left(\lambda\left(b-b^{\prime}\right)+\varphi\right)\right]+\pi H\left[\varphi \beta(1-\theta)+\theta \frac{(1-\beta) \psi}{(1-\theta)}\right] \\
B=r[\theta(1-\pi) \beta+H \beta(1-\theta)]-\pi H \frac{(1-\beta)}{(1-\theta)} \psi \\
C=-(1-\pi) \beta r
\end{gathered}
$$

Similar arguments to Proposition 8 follow.

\section{O Proof of Proposition 15}

Take the function $u\left(\phi_{L}, s\right)$ as given. Then, use the definition of $W^{1}$ and observe that,

$$
W^{2}(B(\omega), u, s)=\max _{d, B^{\prime}} d+\beta E\left[E\left[W^{2}\left(B^{\prime}, u\left(\phi^{\prime}, s\right)\right) \mid s\right] \mid s\right]
$$

subject to

$$
\begin{gathered}
q B(\omega)^{\prime}+d=(q \lambda+M u) B(\omega) \\
B(\omega)^{\prime} \geq 0 \\
d \geq 0
\end{gathered}
$$

One can use the budget constraint to eliminate $d$. One obtains:

$$
W^{2}(B(\omega), u, s)=\max _{B^{\prime}}\left[(q \lambda+M u) B(\omega)-q B(\omega)^{\prime}\right]+\beta E\left[W^{2}\left(B^{\prime}, u\left(s^{\prime}\right), s^{\prime}\right) \mid s\right]
$$

and

$$
B(\omega)^{\prime} \geq 0
$$




$$
\frac{(q \lambda+M u) B(\omega)}{q} \geq B(\omega)^{\prime}
$$

Under the conjecture that the solution is linear we have that:

$$
W^{2}(B(\omega), u, s)=B(\omega) \max _{\frac{B^{\prime}}{B}}\left\{(q \lambda+M u)-q \frac{B(\omega)^{\prime}}{B(\omega)}+\beta E\left[W^{2}\left(u\left(\phi^{\prime}, s\right), s\right) \mid s\right] \frac{B(\omega)^{\prime}}{B(\omega)}\right\}
$$

and

$$
\begin{aligned}
\frac{B(\omega)^{\prime}}{B(\omega)} & \geq 0 \\
\frac{(q \lambda+M u)}{q} & \geq \frac{B(\omega)^{\prime}}{B(\omega)}
\end{aligned}
$$

so since $\frac{B^{\prime}}{B}$ is independent of the size, then,

$$
W^{2}(u, s)=(q \lambda+M u)+\max _{v^{\prime}}\left[\beta E\left[W^{2}\left(u\left(\phi^{\prime}, s\right), s\right) \mid s\right]-q\right] b^{\prime}
$$

The maximization is direct:

$$
B(\omega)^{\prime}=0 \text { if } \beta E\left[W^{2}\left(u\left(\phi^{\prime}, s\right), s\right) \mid s\right]<q
$$

and

$$
B(\omega)^{\prime}=\frac{(q \lambda+M u)}{q} \text { if } \beta E\left[W^{2}\left(u\left(\phi^{\prime}, s\right), s\right) \mid s\right]>q
$$

and otherwise $b^{\prime} \in\left[0, \max \left\{\frac{(q \lambda+u)}{q}, 0\right\}\right]$.

Thus,

$$
W^{2}(u, s)=\max \left\{\beta E\left[W^{2}\left(u\left(\phi^{\prime}, s\right), s\right) \mid s\right], q \lambda+M u\right\}
$$




\section{P Observable Trades}

To begin the analysis, we define an exclusive contract. Firm's observe all trades carried out by agents as well as the quantities of equity they sell and their balance sheet although when they purchase a unit of equity, they cannot distinguish who's equity they are buying. Financial firms will offer competitive contracts. Firms will choose among the set of contracts offered in equilibrium the one that maximizes it's wealth $w_{2, t}$ in the second period. Contracts are made contingent on the agents current't equity $n(z)$. Formally, a contract is composed of several objects,

Definition (Exclusive Feasible Contract). An exclusive contract $c(n)$ is a promise to deliver $x_{c}$ liquid assets in stage 2 , a quantity $e_{c}$ and a recommended function $\Delta e_{c}^{+}(\tilde{z}, z) \in\left[-e_{t+1}^{+}(\tilde{z}, z), 0\right]$ such that

$$
e_{c}=-\int_{0}^{1} \Delta e_{c}^{+}(\tilde{z}, z) d \tilde{z}
$$

Thus, contracts specify a promise to deliver $x_{c}$ units of liquid assets to the entrepreneur in stage 2 that it may use to consume or finance a downpayment for a project. In exchange, the agent is asked to give away a $\Delta e_{t, 1}^{+}(\tilde{z}, z)$ of $\tilde{z}^{\prime} s$ equity. Since, the $\tilde{z}$ is not observable for financial firms, the contract also specifies a total quantity of equity that the entrepreneur will give up. By the revelation principle it is enough to focus in incentive compatible contracts are sufficient to characterize equilibrium contracts. In this context, since aggregate quantities are observable, incentive compatibility is adjusted appropriately to accommodate for deviations among the set of equity sales that amount to the same quantities.

Definition (Incentive Compatibility). A contract $c(n)=\left\{x_{c}, e_{c}, \Delta e_{c}^{+}(\tilde{z}, z)\right\}$ is incentive compatible if

$$
\Delta e_{c}^{+}(\tilde{z}, z)=\arg \max _{\Delta e^{+}(\tilde{z}, z) \in \Delta\left(e_{c}\right)} \int_{0}^{1} \lambda(\tilde{z}) \Delta e^{+}(\tilde{z}, z) d \tilde{z}
$$

where $\Delta\left(e_{c}\right)$ is the set of measurable functions in $[0,1]$ such that,

$$
e_{c}=-\int_{0}^{1} \Delta e^{+}(\tilde{z}, z) d \tilde{z}
$$

Hence, given the quantities $\left(x_{c}, e_{c}\right)$ an incentive compatible is such that the entrepreneurs gives away equity the equity with worse depreciation shock. Thus, an alternative way incentive compatible contracts is by specifying a threshold depreciation $\lambda_{c}$ such that,

$$
e_{c}=-\int_{0}^{1} I_{\left[\lambda(\tilde{z}) \leq \lambda_{c}\right]} e_{t+1}^{+}(\tilde{z}, z) d \tilde{z}
$$

Hence, for the rest of the papers, a contracts will be summarized by the duple $\left(x_{c}, \lambda_{c}\right)$ where $\lambda_{c}$ solves the equation above. Profitable contracts are those in which financial firms make positive profits,

Definition (Incentive Feasible). An incentive compatible contract $\left(x_{c}, \lambda_{c}\right)$ is incentive feasible if,

$$
x_{c}-q_{t} \int_{0}^{1} \lambda(\tilde{z}) I_{\left[\lambda(\tilde{z}) \leq \lambda_{c}\right]} e_{t+1}^{+}(\tilde{z}, z) d \tilde{z} \geq 0
$$

That is, incentive feasible are those contracts that are incentive compatible, feasible, and where financial firms make positive profits. Let $\Gamma \equiv\left\{\left(x_{c}, \lambda_{c}\right) \mid\left(x_{c}, \lambda_{c}\right)\right.$ is incentive feasible $\}$, be the set of incentive feasible contracts. An equilibrium contract solves the following problem, 
Problem 8 (Equilibrium Contracts) An equilibrium contract $\left(x^{*}, e^{*}, \lambda^{*}\right)$ solves

$$
\max _{\left(x_{c}, e_{c}, \lambda_{c}\right) \in \Gamma} r_{t} n_{t}(z)+x_{c}+q_{t}^{R}\left(\lambda(z) k_{t}(z)+\int_{0}^{1}\left(e_{2, t}^{+}(\tilde{z}, z)-e_{2, t}^{-}(\tilde{z}, z)\right) d \tilde{z}\right)
$$

subject to

$$
e_{2, t}^{+}(\tilde{z}, z)=\lambda(\tilde{z})\left(\Delta e_{c}^{+}(\tilde{z}, z)+e_{1, t}^{+}(\tilde{z}, z)\right)
$$

The following proposition characterizes optimal contracts when trades are observable.

Proposition 21 (Observable Trades) With observable trades, an equilibrium competitive contract recovers first best outcomes.

\section{P.1 Proof of Proposition 21}

$$
\begin{aligned}
& \max _{\left(x_{c}, \lambda_{c}\right) \in \Gamma} x_{c}+q_{t}^{R} \int_{0}^{1} \Delta e_{c}^{+}(\tilde{z}, z) d \tilde{z} \\
= & \max _{\left(x_{c}, \lambda_{c}\right) \in \Gamma} x_{c}-q_{t}^{R} \int_{0}^{1} I_{\left[\lambda(\tilde{z}) \leq \lambda_{c}\right]} e_{t+1}^{+}(\tilde{z}, z) d
\end{aligned}
$$

and subject to

$$
x_{c}-q_{t} \int_{0}^{1} \lambda(\tilde{z}) I_{\left[\lambda(\tilde{z}) \leq \lambda_{c}\right]} e_{t+1}^{+}(\tilde{z}, z) d \tilde{z} \geq 0
$$

where combining the constraints inside leads to,

$$
\max _{\left(x_{c}, \lambda_{c}\right) \in \Gamma}\left(q_{t}-q_{t}^{R}\right) \int_{0}^{1} \lambda(\tilde{z}) I_{\left[\lambda(\tilde{z}) \leq \lambda_{c}\right]} e_{t+1}^{+}(\tilde{z}, z) d \tilde{z}
$$

so the optimal solution is the one that makes $\lambda_{c}$ as large as possible. Assume such solution is $\lambda_{c}=1$, then the solution is given by

$$
x_{c}=q_{t} \int_{0}^{1} \lambda(\tilde{z}) e_{t+1}^{+}(\tilde{z}, z) d \tilde{z}
$$

which amounts to the case with full liquidity.

\section{Q Accounting Identities}




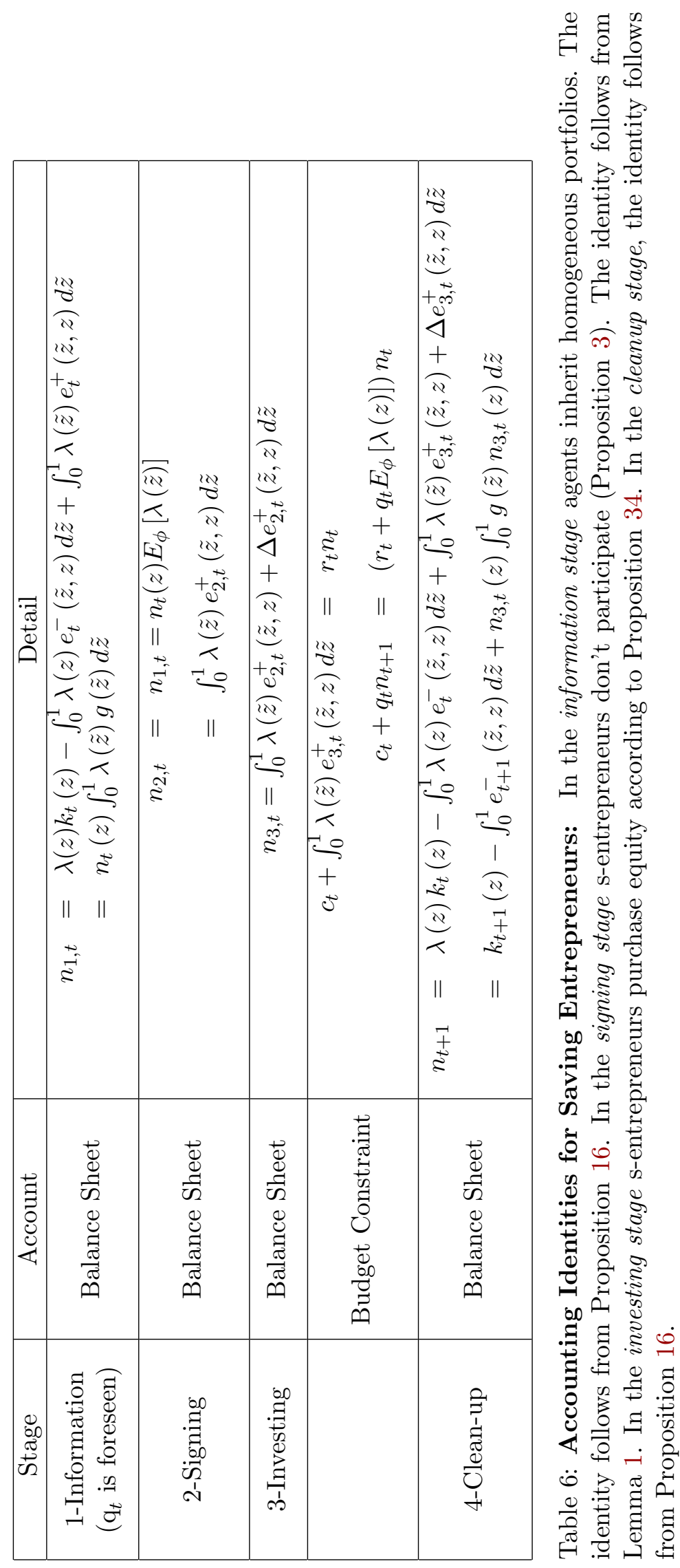




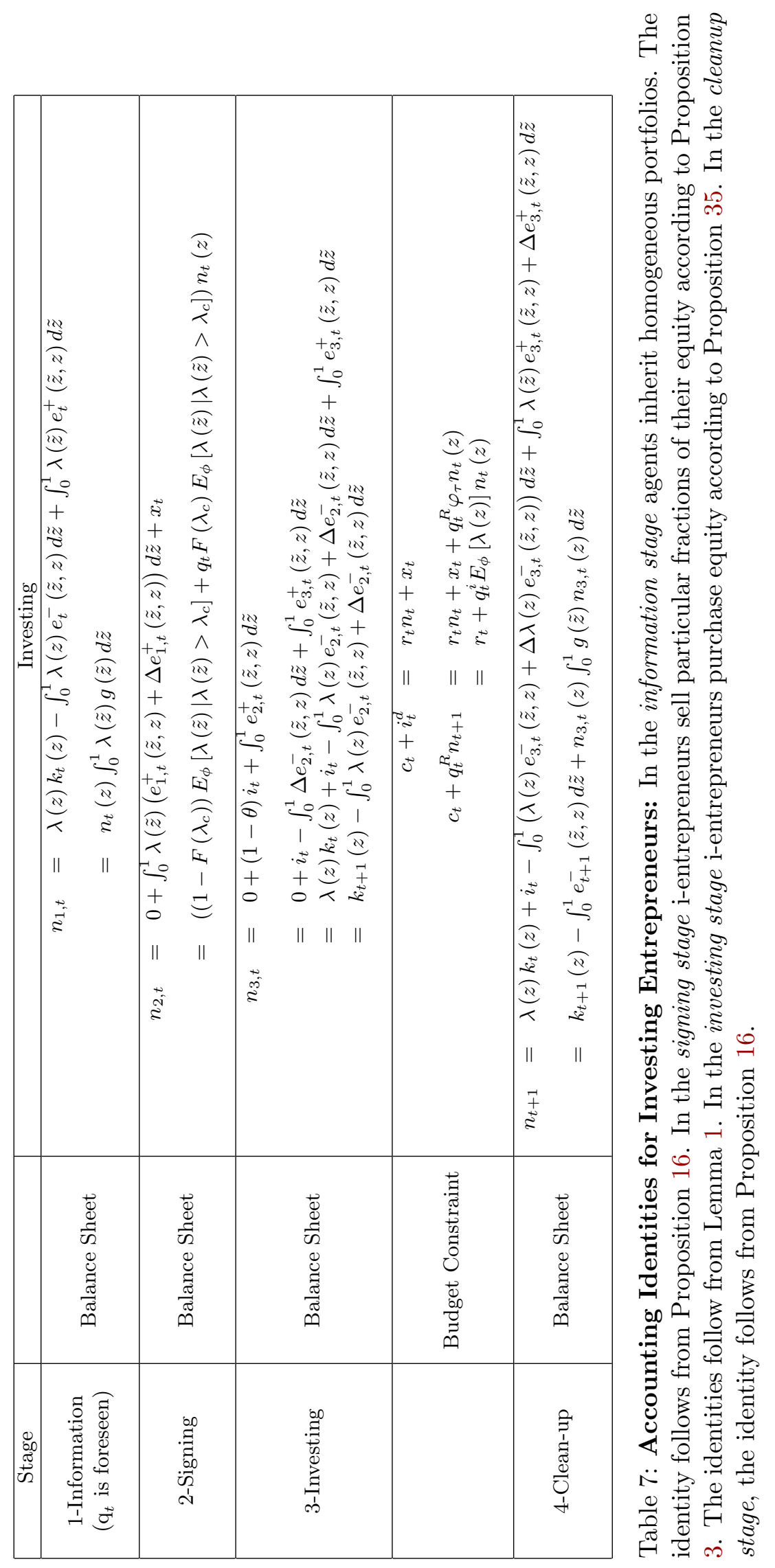


R Additional Figures 


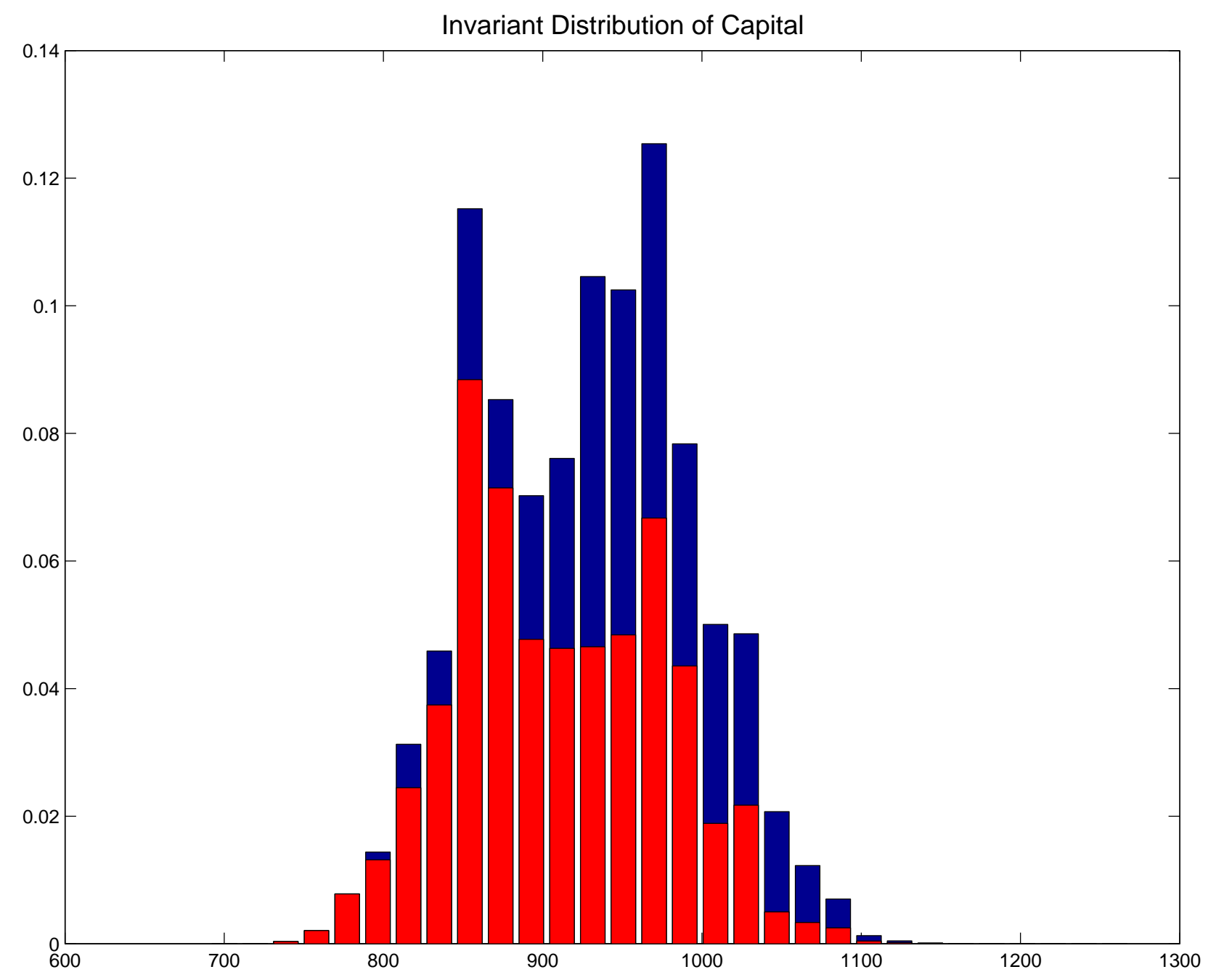

Figure 15: Invariant Distributions. The figure shows the invariant distribution of capital the capital stock. 